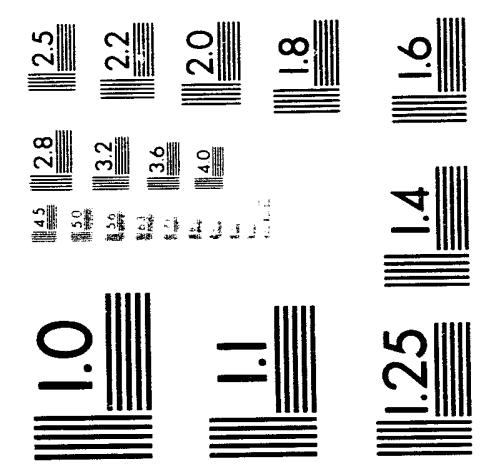



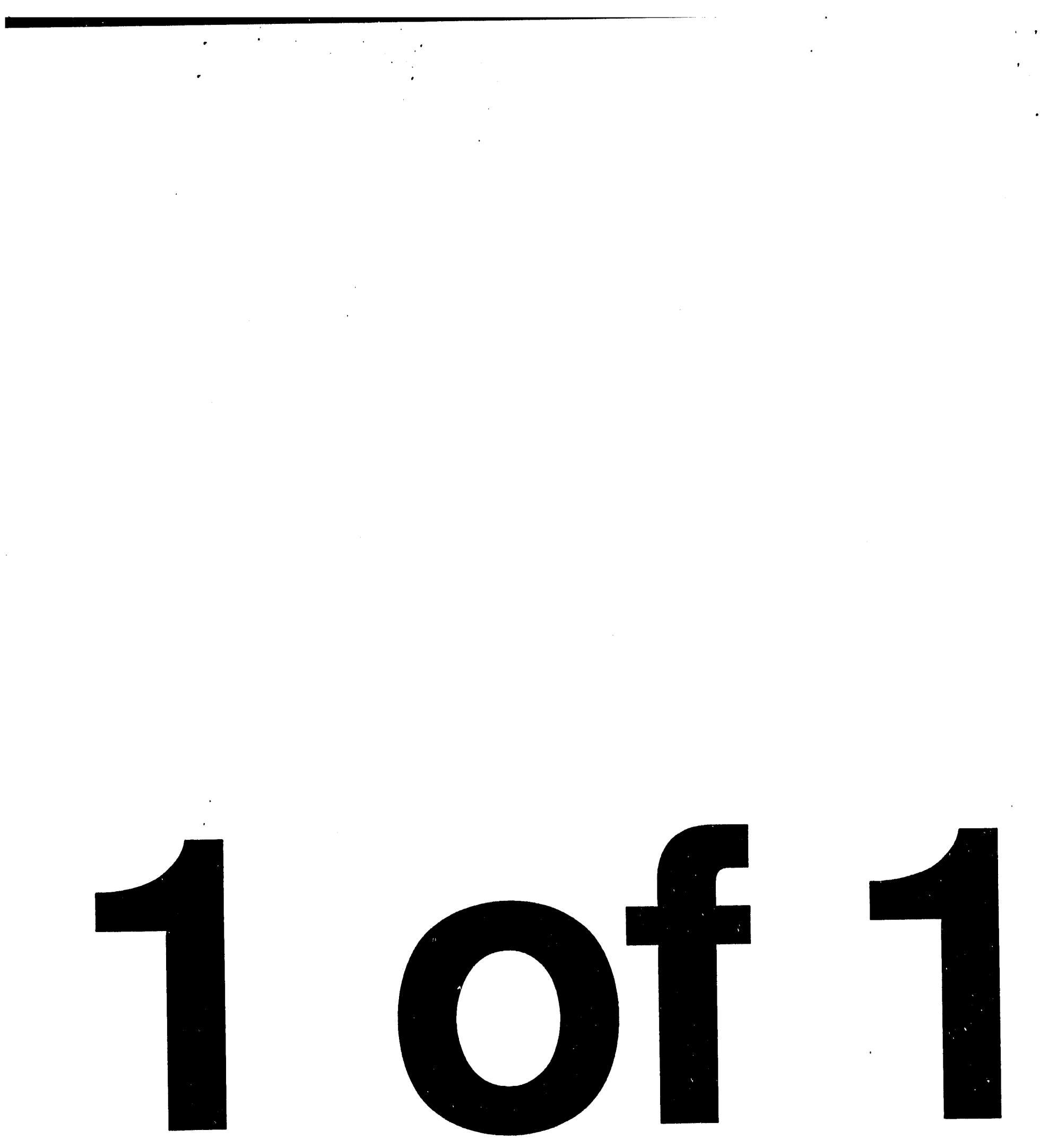
DOE/EIA-0535(92)

Distribution Category UC-950

\title{
Fuel Oil and Kerosene Sales 1992
}

\section{October 1993}

\author{
Energy Information Administration \\ Office of Oil and Gas \\ U.S. Department of Energy \\ Washington DC 20585
}




\section{Contacts}

The Fuel Oil and Kerosene Sales 1992 report is prepared by the Energy Information Administration (EIA) under the general direction of Michael Griffey (202) 586-9601, Petroleum Marketing Division, Office of Oil and Gas.

Detailed, technical questions about tables referencing the form EIA-821 may be directed to:

Alice Lippert (202) 586-9600

\section{Fuel Oil and Kerosene Data Available on Diskette}

Annual petroleum marketing data are available on a diskette which contains statistics from the Fuel Oil and Kerosene Sales 1992 report. Included are annual sales data on petroleum volumes of kerosene, distillate fuel oils, and residual fuel oils by State. Annual historical data at the national level are provided in summary tables.

The Fuel Oil and Kerosene Sales diskette is in ASCII text fornat on a double-sided low-density 5.25 inch diskette for use with IBM-compatible personal computers.

\section{How to Order}

Fuel oil and kerosene sales data are available on diskette from:

Superintendent of Documents

U.S. Government Printing Office

Attn: Queenie Faison

P.O. Box 37082

Washington, DC 20013

(202) 512-1530

National Technical Information Service (NTIS)

Office of Data Base Services

U.S. Department of Commerce

5285 Port Royal Road

Springfield, Virginia 22161

(703) $487-4650$

Further information as to content may be obtained from the National Energy Information Center (NEIC), telephone (202) 586-8800. 


\section{Contents}

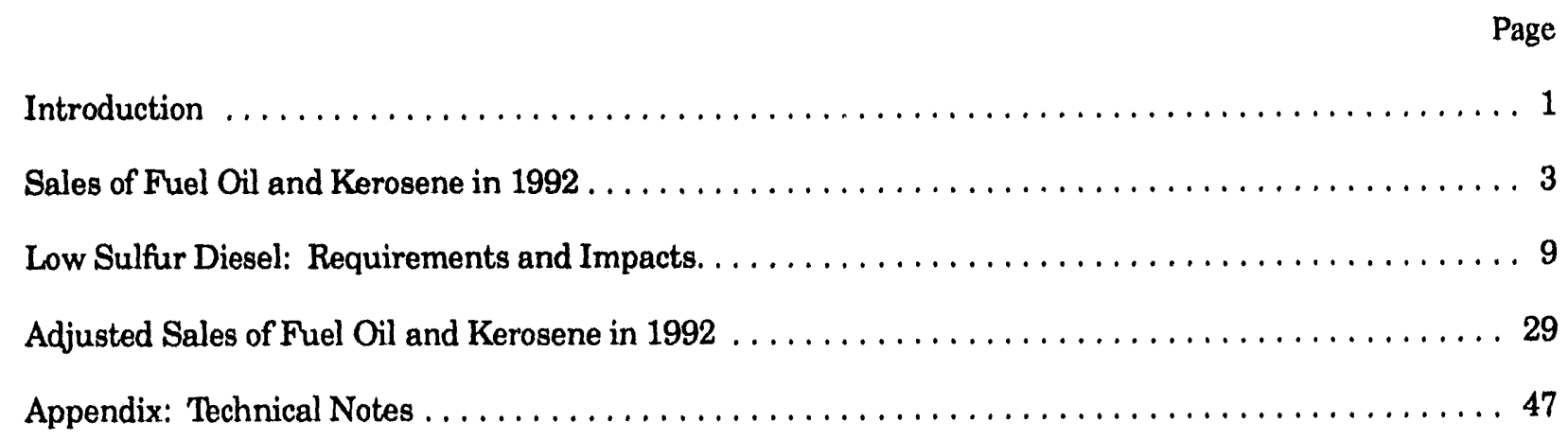

\section{Figures}

1. Changes in the Color of Diesel Fuel on October $1,1993 \ldots \ldots \ldots \ldots \ldots \ldots \ldots \ldots \ldots$ 


\section{Tables}

\section{Sales of Fuel Oll and Kerosene In 1992}

1. Sales of Distillate Fuel Oil by End Use in the United States: $1988-1992 \ldots \ldots \ldots \ldots \ldots \ldots \ldots$

2. Sales of Residual Fuel Oil by End Use in the United States: $1988-1992$. . . . . . . . . . . . . . . 14

3. Sales of Kerosene by End Use in the United States: $1988-1992 \ldots \ldots \ldots \ldots \ldots \ldots \ldots \ldots \ldots 14$

4. Sales of Distillate Fuel Oil by End Use, 1991 and $1992 \ldots \ldots \ldots \ldots \ldots \ldots \ldots \ldots \ldots$

5. Sales of Residual Fuel Oil by End Use, 1991 and $1992 \ldots \ldots \ldots \ldots \ldots \ldots \ldots \ldots$

6. Sales of Kerosene by End Use, 1991 and $1992 \ldots \ldots \ldots \ldots \ldots \ldots \ldots \ldots \ldots \ldots \ldots$

7. Sales for Residential Use: Distillate Fuel Oil and Kerosene, $1992 \ldots \ldots \ldots \ldots \ldots \ldots \ldots \ldots$

8. Sales for Commercial Use: Distillate Fuel Oil, Residual Fuel Oil and Kerosene, 1992 . . . . . . . . 24

9. Sales for Industrial Use and for Oil Company Use: Distillate Fuel Oil, Residual Fue]

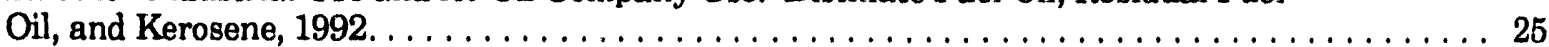

10. Sales for Farm Use: Distillate Fuel Oil and Kerosene; Sales for Electric Utility Use:

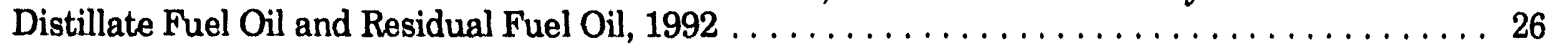

11. Sales for Transportation Use: Distillate Fuel Oil and Residual Fuel Oil, $1992 \ldots \ldots \ldots \ldots \ldots$

12. Sales for Military, Off-Highway, and All Other Uses: Distillate Fuel Oil, Residual Fuel

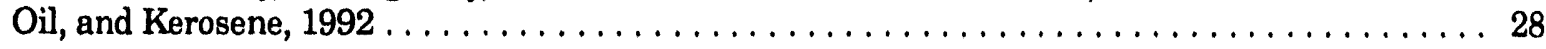

\section{Adjusted Sales of Fuel Oil and Kerosene in 1992}

13. Adjusted Sales of Distillate Fuel Oil by End Use in the United States: $1988-1992$. . . . . . . . . 30

14. Adjusted Sales of Residual Fuel Oil by End Use in the United States: $1988-1992 \ldots \ldots \ldots \ldots$. . . . 31

15. Adjusted Sales of Kerosene by End Use in the United States: $1988-1992 \ldots \ldots \ldots \ldots \ldots \ldots$

16. Adjusted Sales of Distillate Fuel Oil by End Use, 1991 and $1992 \ldots \ldots \ldots \ldots \ldots \ldots \ldots$ 
17. Adjusted Sales of Residual Fuel Oil by End Use, 1991 and $1992 \ldots \ldots \ldots \ldots \ldots \ldots$

18. Adjusted Sales of Kerosene by End Use, 1991 and $1992 \ldots \ldots \ldots \ldots \ldots \ldots \ldots$

19. Adjusted Sales for Residential Use: Distillate Fuel Oil and Kerosene, $1992 \ldots \ldots \ldots \ldots \ldots$. . . . 40

20. Adjusted Sales for Commercial Use: Distillate Fuel Oil, Residual Fuel Oil and

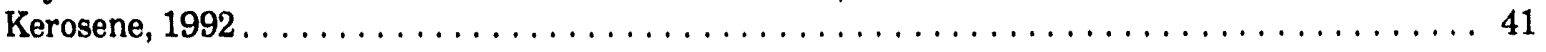

21. Adjusted Sales for Industrial and Oil Company Use: Distillate Fuel Oil, Residual

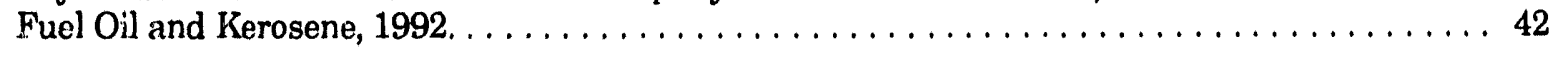

22. Adjusted Sales for Farm Use: Distillate Fuel Oil and Kerosene; Adjusted Sales for Electric Utility Use: Distillate Fuel Oil and Residual Fuel Oil, $1992 \ldots \ldots \ldots \ldots \ldots \ldots \ldots \ldots . \ldots 3$

23. Adjusted Sales for Transportation Use: Distillate Fuel Oil and Residual Fuel Oil, 1992 . . . . . . 44

24. Adjusted Sales for Military, Off-Highway, and All Other Uses: Distillate Fuel Oil, Residual Fuel Oil, and Kerosene, $1992 \ldots \ldots \ldots \ldots \ldots \ldots \ldots \ldots \ldots \ldots \ldots \ldots \ldots \ldots$ 


\section{Introduction}

This publication contains the 1992 survey results of the "Annual Fuel Oil and Kerosene Sales Report" (Form EIA-821). This is the fourth year that the survey data have appeared in a separate publication. Prior to the 1989 report, the statistics appeared in the Petroleum Marketing Annual (PMA) for reference year 1988 and the Petroleum Marketing Monthly (PMM) for reference years 1984 through 1987. The 1992 edition marks the ninth annual presentation of the results of the ongoing "Annual Fuel Oil and Kerosene Sales Report" survey.

Except for the kerosene and on-highway diesel information, data presented in Tables 1 through 12 (Sales of Fuel Oil and Kerosene) present results of the EIA-821 survey. Tables 13 through 24 (Adjusted Sales of Fuel Oil and Kerosene) include volumes that are based on the EIA-821 survey but have been adjusted to equal the products supplied volumes published in the Petroleum Supply Annual (PSA).

Tables 1. through 12 contain sales estimates resulting from the EIA-821 survey for all categories except kerosene and on-highway diesel. For on-highway diesel the survey was not expected to yield valid statistics because the sampling frame does not include a comprehensive listing of all truck stops. Hence, State-level data obtained from the Federal Highway Administration were used instead.

Kerosene sales data were not expected to be complete because a comprehensive listing of kerosene retailers was not evailable to serve as a sampling frame. It was thought that a complete frame would be expensive to construct because many kerosene retailers are convenience stores or other small businesses. Because of these concerns, kerosene sales data have been published only after adjusting the sales data so that they add to the U.S. total of kerosene product supplied from the PSA.

In Tables 13 through 24, estimates of distillate fuel oil are adjusted at the Petroleum Administration for Defense (PAD) district level to equal published PSA volume estimates of products supplied. For certain sales categories, data obtained from alternate sources are used instead of the adjusted numbers. See "Technical Note 3 " in Appendix for further explanation.

Kerosene and residual fuel oil in Tables 13 through 24 are adjusted at the national level to equal published
PSA products supplied estimates. Historically, kerosene estimates have always been adjusted at the PAD district level. However, in 1992, certain kerosene estimates at the $\mathrm{PAD}$ district level resulted in negative numbers. Hence, the adjustment procedure was changed to display sales volumes of kerosene for all PAD districts.

The adjustment procedure for residual fuel oil was changed in 1990. In previous years, estimates of vessel bunkering volumes were obtained from the Bureau of the Census report, United States Foreign Trade, Bunker Fuels. This Census report was discontinued after 1989. In order to minimize disruption to the residual fuel oil adjusted sales data series, the Census data were removed from prior years' estimates and the adjustment to equal products supplict is now performed at the national level.

The sales data (Tables 1 through 12) differ from the adjusted sales estimates (Tables 13 through 24) for many $r$ isons, including:

- Some products are interchangeable (fungible) and may be supplied as one product and sold as another product. For example, kerosene, kerosene-type jet fuel, and No. 1 fuel oil can be used interchangeably.

- Products supplied into a PAD district may be blended prior to final sale. For example, residual fuel and No. 2 distillate may be blended and sold as No. 4 fuel oil or, in colder climates, kerosene may be blended with distillate fuel oil and sold as heating oil.

- Geographic differences can be attributed to the transportation of product by truck or rail from the district of production. Inter-PAD district movements of products by these modes of transportation are not accounted for in Tables 13 through 24.

- Products may be supplied into a PAD district but the final sale may cross PAD district boundaries. For example, a fuel oil dealer in Ohio (PAD District II) may make retail sales into Pennsylvania (PAD District IB) and/or West Virginia (PAD District IC).

- Drawdowns or buildups in stocks will cause volumes supplied to diffar from sales volumes. 


\section{Highlights}

\section{Sales of Fuel Oil and Kerosene in 1992}

Lower petroleum prices, a colder winter, and a recovering economy increased demand for distillate fuel oil in 1992. After showing a decline for the previous 2 years, distillate fuel oil sales rose 4.5 percent in 1992. Demand for residual fuel oil, on the other hand, diminished, particularly in the electric utility sector, due to the coolest summer in 77 years (U. S. Department of Commerce, National Oceanic and Atmospheric Administration, Climate Analysis Center) and competition from natural gas. Residual fuel oil purchases dropped 8.1 percent in 1992. Sales of kerosene also fell after showing an increase in 1991.

In 1992, a total of 64.1 billion gallons of fuel oil and kerosene was sold in the United States, an increase of 0.9 percent over 1991. Distillate fuel oil's share of these products increased to 73.7 percent, while residual fuel oil and kerosene products' share fell to 25.3 and 1.0 percent, respectively. In 1991, these products' shares of total sales were $71.1,27.8$, and 1.1 percent, respectively.

\section{Distillate Fuel Oil}

The transportation sector rebounded in 1992 with distillate sales increasing 5.3 percent over 1991 . A slowly improving economy spurred demand for more goods that in turn increased demand for freight transportation services. This was evidenced by increased sales of on-highway diesel, 21.4 billion gallons, 890 million gallons over 1991. Similarly, the vessel bunkering and railroad industries displayed sales volumes of 2.2 and 3.2 billion gallons, a jump of 8.4 and 10.2 percent, respectively, over 1991 . The transportation sector's share of total distillate sales accounted for 56.6 percent, with 45.2 percent coming from on-highway diesel sales (Table HL1).

Distillate sales to residential customers for home heating increased over 1991. Overall, the average temperature in 1992 was almost 5.0 percent colder than in 1991 and prices of residential distillate averaged 8.3 cents a gallon less than during the previous year (Short-Term Energy Outlook, August 1993 and Petroleum Market. ing Annual 1992). Thus, heating demand for this end-use sector was up 7.6 percent. Commercial distillate sales remained constant during 1992. Of note, the price gap between residential heating oil and natural gas also narrowed in 1992.

Farmers purchased 10.8 percent more distillate in 1992 than in 1991. The largest increase in sales occurred in the Midwest where distillate fuel oil

Table HL1. Volume Distribution of Distillate and Residual Fuel Olls, 1991 and 1992

\begin{tabular}{|c|c|c|c|c|c|c|c|c|}
\hline \multirow[b]{2}{*}{ End Use } & \multicolumn{2}{|c|}{ Distillate 1992} & \multicolumn{2}{|c|}{ Distillate 1981} & \multicolumn{2}{|c|}{ Resldual 1982} & \multicolumn{2}{|c|}{ Resldual 1991} \\
\hline & $\begin{array}{l}\text { Volume } \\
\text { (million } \\
\text { gallons) }\end{array}$ & $\begin{array}{c}\text { Percent } \\
\text { Share }\end{array}$ & $\begin{array}{l}\text { Volume } \\
\text { (million } \\
\text { gallons) }\end{array}$ & $\begin{array}{c}\text { Percent } \\
\text { Share }\end{array}$ & $\begin{array}{l}\text { Volume } \\
\text { (million } \\
\text { gallons) }\end{array}$ & $\begin{array}{c}\text { Percent } \\
\text { Share }\end{array}$ & $\begin{array}{l}\text { Volume } \\
\text { (million } \\
\text { gallons) }\end{array}$ & $\begin{array}{c}\text { Percent } \\
\text { Share }\end{array}$ \\
\hline Residential ...................... & 7,291 & 15.4 & 6,776 & 15.0 &.. & .. & . & . \\
\hline 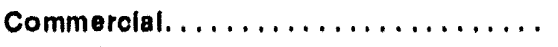 & 3,771 & 8.0 & 3,768 & 8.3 & 1,176 & 7.3 & 1,424 & 8.1 \\
\hline 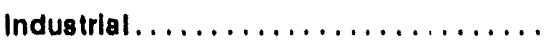 & 2,312 & 4.9 & 2,309 & 5.1 & 2,127 & 13.1 & 1,935 & 11.0 \\
\hline 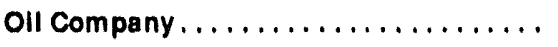 & 664 & 1.4 & 739 & 1.6 & 271 & 1.7 & 301 & 1.7 \\
\hline Farm $\ldots \ldots \ldots \ldots \ldots \ldots \ldots \ldots \ldots \ldots \ldots \ldots \ldots$ & 3,500 & 7.4 & 3,158 & 7.0 & .. & -. & $\cdot$ & - \\
\hline Electric $\ldots \ldots \ldots \ldots \ldots \ldots \ldots \ldots \ldots$ & 541 & 1.1 & 595 & 1.3 & 5,961 & 36.8 & 7,071 & 40.1 \\
\hline 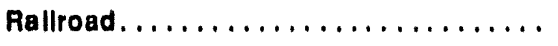 & 3,173 & 6.7 & 2,879 & 6.4 & .. & -. & $\cdot$ & \\
\hline 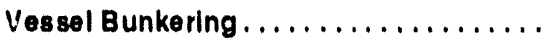 & 2,219 & 4.7 & 2,046 & 4.5 & 6,563 & 40.5 & 6,773 & 38.4 \\
\hline On-Highway $\ldots \ldots \ldots \ldots \ldots \ldots \ldots$ & 21,375 & 45.2 & 20,485 & 45.3 & .. & $\cdots$ & $\cdot$ & . \\
\hline 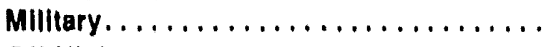 & 653 & 1.4 & 812 & 1.8 & 92 & 6 & 120 & .7 \\
\hline 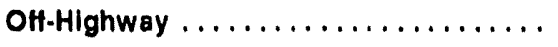 & 1,758 & 3.7 & 1,642 & 3.6 & $\cdots$ & $\cdots$ & - & - \\
\hline Other $\ldots \ldots \ldots \ldots \ldots \ldots \ldots \ldots \ldots \ldots \ldots \ldots$ & 6 & .0 & 2 & .0 & 9 & .1 & 8 & 0 \\
\hline Total .. & 47,262 & 100.0 & 45,211 & 100.0 & 16,199 & 100.0 & 17,632 & 100.0 \\
\hline
\end{tabular}

Sources: Energy Information Administration, Form ElA 821, "Annual Fuel Oil and Kerosene Sales Report," 1991 and 1992. 
demand increased 15.6 percent. The increases were caused by a colder heating season and an abundant corn harvest. According to the National Agricultural Statistics Service (NASS), corn production and acreage yields were up 26.8 and 21.0 percent, respectively, over 1991 (Annual Crop Summary, January 1993). Not only were more acres of corn harvested, but each acre planted yielded more corn. Additionally, NASS farm diesel expenditures were up as well--13.5 percent.

Off-highway distillate sales, i.e., sales for construction and road building, were up 7.1 percent in 1992. Residential housing starts were up as well as expenditures for Federal highway construction projects. According to the Federal Highway Administration (Office of Highway Information Management), Federal funds for highway construction increased 4.5 percent in 1992, up 1.4 billion dollars.

Not all end-use sectors showed increases in 1992, however. For example, military sales were off 19.6 percent from 1991. Base closings and the end of the Gulf War (Desert Storm) accounted for this decrease. Oil company use sales also dropped as evidenced by the drop in oil rig counts in 1992 . Not only did the number of rotary oil rigs in operation decrease by 22.6 percent, but total footage drilled for all wells fell 16.3 percent (Monthly Energy Review, August 1993).

Industrial distillate sales remained constant in 1992, even though industrial production was up 2.3 percent (Economic Indicators, June 1993). The primary reason for the lack of change in distillate fuel use was the extremely low price of natural gas, particularly in the first half of 1992. Natural gas delivered to industrial customers was up 6.0 percent in 1992 (Monthly Energy Review, August 1993).

Distillate fuel oil sales increased in all PAD districts in 1992, except PAD District V which remained constant (Figure HL2). The largest increase occurred in PAD District IA, the Northeast, with a 10.2 percent increase over 1991. On a market share basis, PAD District I, the East Coast, and PAD District II, the Midwest, both showed increases. The remaining PAD districts' shares of total distillate fuel oil fell slightly, with PAD District V, the West Coast, declining 0.6 percentuge points.

\section{Residual Fuel Oil}

Residual fuel oil sales declined for the third straight year. A total of 16.2 billion gallons of residual fuel oil was sold in the United States in 1992, compared to 17.6 billion gallons in 1991 (Table HL1). Sales declines were attributed to a cooler than normal summer, the introduction of the Iroquois natural gas pipeline from Canada into the northeast region of the United States, competitive natural gas prices, and Clean Air Act legislation (American Petroleum Institute's (API) Monthly Statistical Report, January 13, 1993).

Residual fuel oil sales share of total fuel oil sales fell in 1992, primarily due to the decline of electric utility sales. For the third consecutive year, electric utilities purchased less residual fuel oil, down 15.7 percent from 1991. Electric utility demand was down because the summer of 1992 was the coolest since 1915 and was also 21.6 percent milder than 1991 (Short-Term Energy Outlook, August 1993). Debris (stratospheric aerosols) from the Mt. Pinatubo volcano contributed to this weather phenomenon (Fourth Annual Climate Assessment 1992, National Oceanic and Atmospheric Administration).

An additional factor contributing to the fall in demand of residual fuel oil was the continuing price competition from natural gas. Although natural gas prices were higher in 1992 than in 1991, prices to alectric utilities were still less than residual fuel oil, therefore, enticing utilities to switch to this cheaper fuel. The abundance of natural gas from the lroquois pipeline also made it easier for utilities in the northeast to consume more natural gas in 1992 (APl's Monthly Statistical Report, January 13,1993$)$.

The shipping industry bought 3.1 percent less residual fuel oil in 1992. Although waterborne activity was up in the United States, the effect of the bunkering tax in California, which took effect in July 1991, more than offset any increases in demand for bunker fuel. (Note: the bunkering tax was repealed in July 1992; however, the new tax law was not effective until January 1993.) Bunkering sales fell for the second straight year in California, with sales dropping 29.5 percent from 1991 , almost doubling last year's sales decrease when residual fuel oil purchases fell 18.0 percent in this State.

While industrial residual fuel oil sales increased 9.9 percent (192.1 million gallons) in 1992, oil company use sales decreased 9.9 percent (29.9 million gallons). 'Though the industrial sector has used natural gas in the past because it has been, on average, a less costly fucl than residual fuel oil, a slightly stronger economy and higher industrial natural gas prices in 1992 caused the industrial sector to purchase residual fuel oil to produce domestic goods (Monthly Energy Review, August 1993). Oil companies used less residual fuel oil in refinery operations. Although refinery utilization rates increased from 89.2 percent in 1991 to 91.6 percent in 1992, refinery cupacity fell 1.6 percent (Petroleum Supply Annual 1992).

Residual fuel oil sales to the military declined by 23.5 percent in 1992. This sigmificant drecease was attributed to the end of the Culf War. Commercial sales also showed a drop of 17.4 percent. 
As in past years, residual fuel oil sales remained concentrated in PAD District I, the East Coast (Figure HL2). Virtually all of the residual fuel was used in this region for electric utility, industrial, commercial, and vessel bunkering uses. The East Coast accounted for the vast majority of electric utility sales, 90.0 percent, an increase over 1991 of 0.6 percent (Figure HL3). PAD District I's share of total residual fuel oil decreased, however, from 60.8 percent in 1991 to 60.2 in 1992. PAD District III, the Gulf Coast, showed an increase in its share of residual fuel oil sales of 1.3 percent. The latter increase was partly due to an increase of vessel bunkering sales in this area of 1.7 percent.

\section{Kerosene}

Krrosene sales fell 10.3 percent in 1992 after rebounding in 1991. The bulk of kerosene sales were made to the residential sector, with this sector's share of total kerosene remaining basically unchanged from 1991, at 75.7 percent. All end-use sectors showed sales decreases in 1992, with the exception of farm use where sales increased 17.5 percent. 'The majority of kerosene sales were made in PAD District 1--77.0 percent.
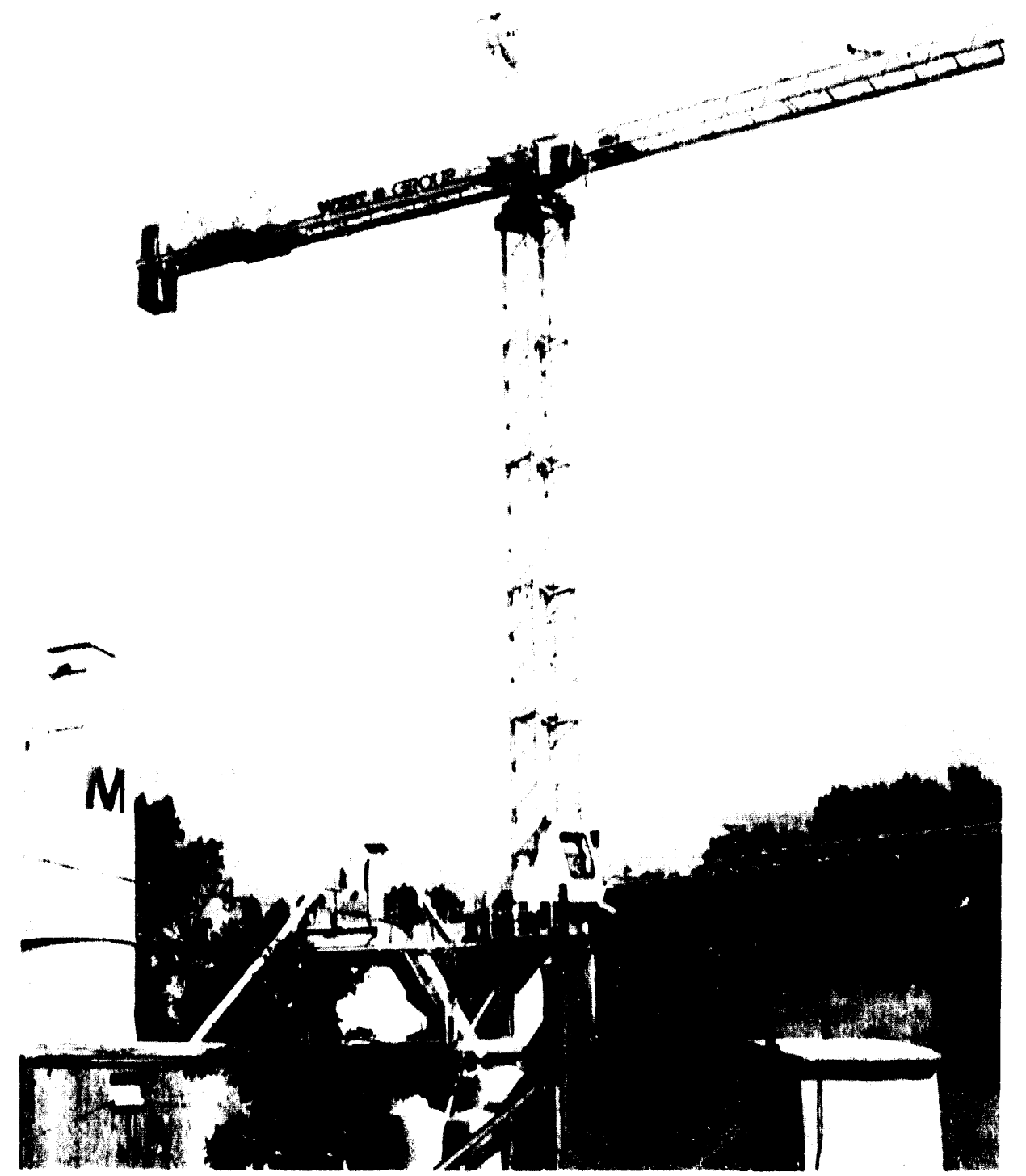

Distillate sales for construction and road building, i.e. off-highway use, were up) 116 million gallons (7.1 percent) in 1992 . 
Figure HL1. U.S. Sales of Distillate and Rer,idual Fuel Olls by End Use, 1990-1992
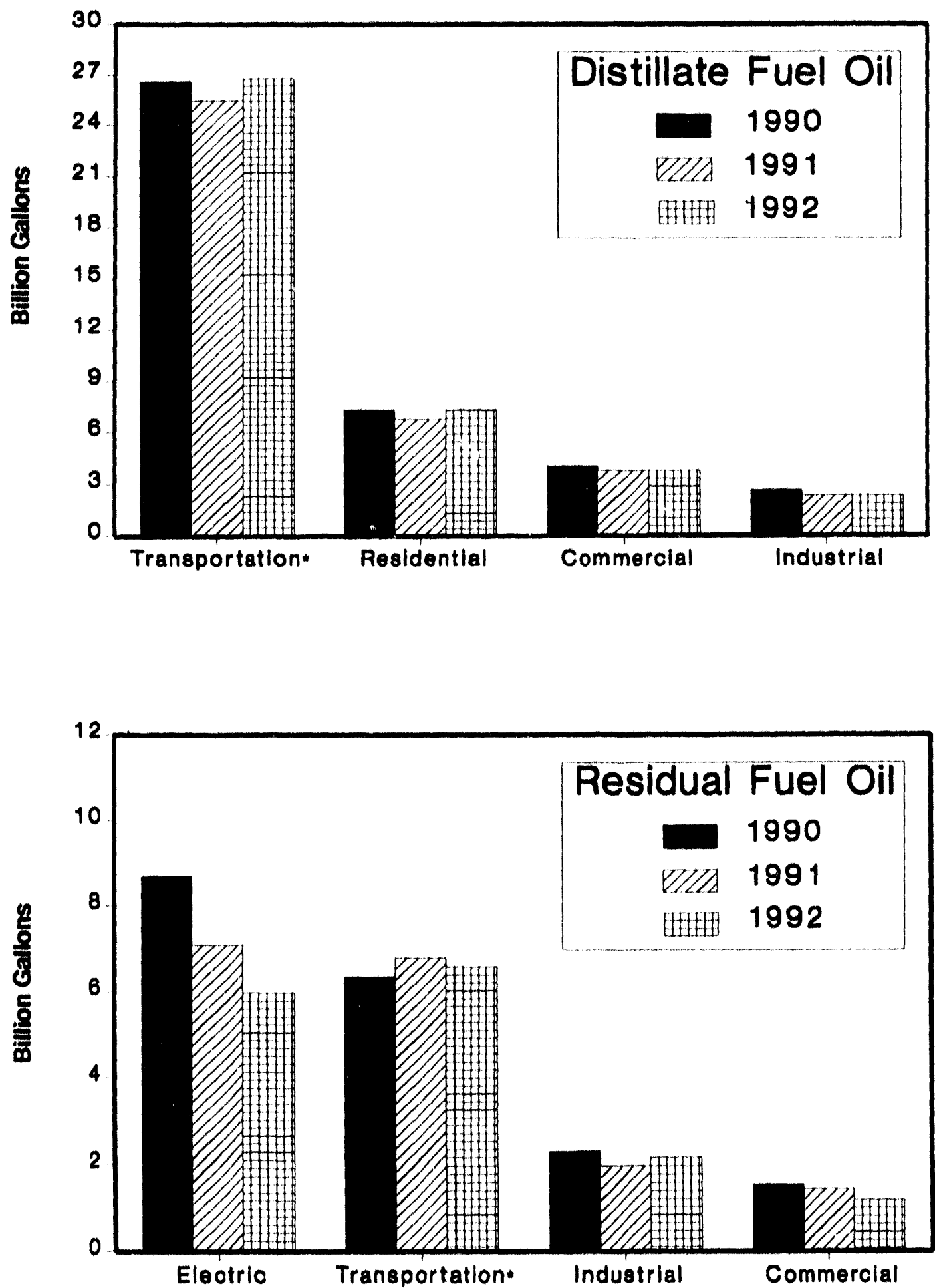

- For distillate fuel oll, transportation use comprises railroad, vessel bunkering, and on-highway diesel end-use categories. For residual fuel oll, transportation use comprises the vessel bunkering end-use category Sources: Energy Information Administration, Form E|A-821. "Fuel Oll and Kerosene Sales Report," 1991 and 1992 

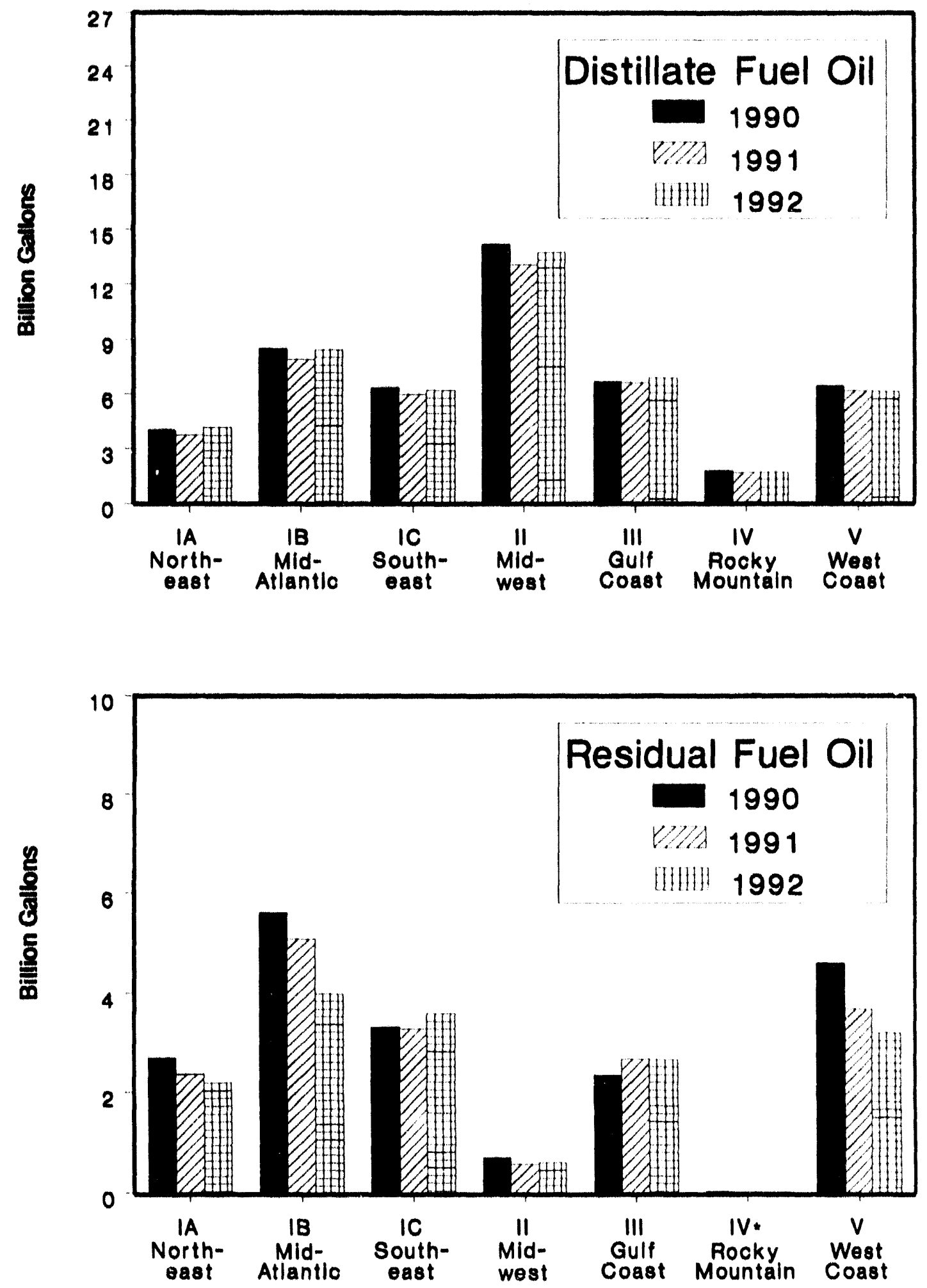

- Residual fuel oil sales in PAD Distrlot IV were too small to appear in this graph.

Sources: Energy Information Administration, Form EIA-821, "Fuel Oll and Kerosene Sales Report," 1991 and 1992. 
Figure HL3. Distillate and Residual Fuel Oil Sales for Selected End-Use Categories, by PAD District Distillate: Transportation

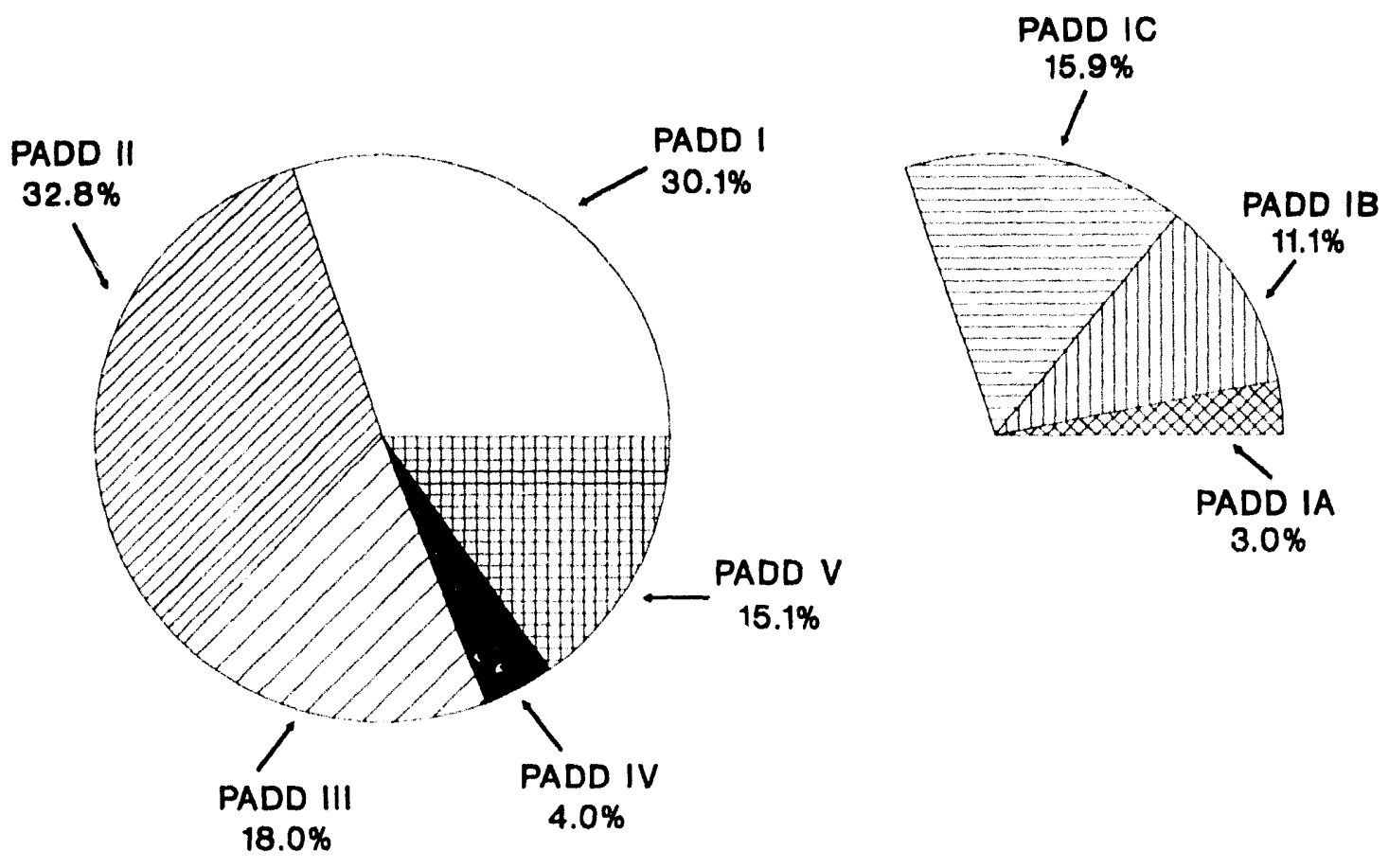

\section{Residual: Electric Utility}

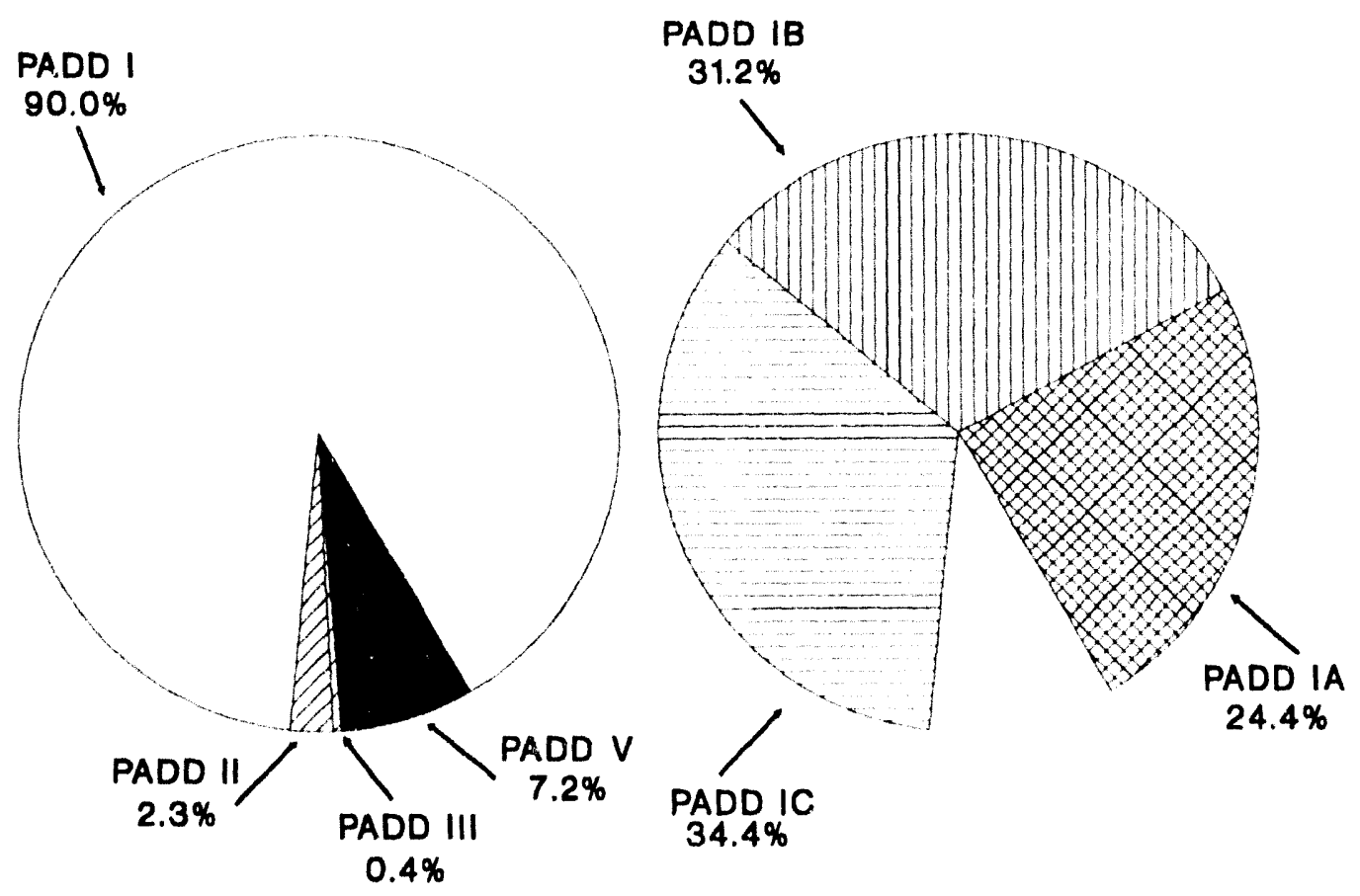

Source: Energy Information Administration, Form ElA-821, "Fuel Oil and Kerosene Sales Report," 1992. 


\section{Low Sulfur Diesel: Requirements and Impacts}

\section{Background}

After several years of study, the Environmental Protection Agency (EPA) proposed to regulate the sulfur content for highway diesel fuel. A primary reason for proposing lower sulfur highway diesel fuel was to allow proper operation of emission control equipment on 1994 and later motor vehicles. The regulations are intended to reduce the sulfur content in highway diesel fuels by 80 percent on average, while capping aromatics at current levels.

\section{EPA Requirements}

The final rule approved in 1992 states that "effective October 1, 1993, all highway diesel fuel is required to comply with the maximum sulfur content standard of 0.05 percent by weight" and must "meet either the 40 cetane index minimum or 35 volume percent aromatic maximurn." These limits on sulfur and aromatics were initially proposed jointly by members of the diesel engine manufacturing and the petroleum refining industries back in $1989 .{ }^{2}$ The regulation "includes, but is not limited to, any diesel fuel, fuel oil, furnace oil, heating oil, kerosene, jet fuel, JP-4, JP-5, JP-8, marine diesel or railroad diesel that is suitable for use as a diesel motor vehicle fuel or is suitable for blending with diesel motor vehicle fuel." ${ }^{3}$ EPA requires that blue dye 1,4 - dialkylamino-anthraquinone be added to any diesel fuel or fuel suitable for blending with diesel motor vehicle fuel which is not in compliance with the sulfur and cetane standards. The blue dye will be added at the refinery for domestic production and at terminals for imports.

The blue dye added to the diesel fuel will not necessarily make the fuel appear blue and is not intended to make it blue. An unspecified amount must simply be added. The dye is intended to change the color of the high sulfur diesel to make it "noticeably different" from the low sulfur diesel. With the variety of current colors and some of the exceptions to the requirements, color by itself will not determine a violation according to the EPA; color will only indicate that testing is warranted.

\section{Exceptions to Rules on Dyeing}

High sulfur diesel used in refinery operations or as feedstocks does not need blue dye added. Even high sulfur diesel moving from one refinery to another does not need to be dyed if the diesel is for feedstock or refinery use and is segregated and documented as such during shipment.

The blue ciye will not be added to high sulfur kerosene based jet fuels if there is a downstream use for the fuel in aircraft, if the fuel is segregated, and if it is documented as high sulfur kerosene based jet fuel. The reason kero-jet is not being dyed is to avoid confusion between it and some grades of aviation gasoline. One grade of aviation gasoline is blue in color; another is green.

According to Defense Fuel Supply Center (DFSC) officials, the current Department of Defense (DOD) plan calls for all on-highway diesel used by the U.S. military in the United States to comply with the low sulfur requirements. On August 20, 1993, EPA did grant DOD's request that high sulfur marine diesel supplied by the DFSC be exempt from the blue dye requirement if the fuel is clearly marked and segregated. The exemption is based on DOD's concern that the dye may cause stability problems in the fuel when it is in long-term storage.

\footnotetext{
'See Environmental Protection Agency's (El'A) final rule in the lederal Register on May 7, 1992, p. 19535 or El'A's "1993 Diesel Desulfurization Question and Answer Document," August 5, 1993, p. 9. A notice of proposed decision to exempt Alaska has been published in the Federal Register, August 27, 1993.

${ }^{2}$ Federal Register, August 24, 1989, p. 35277.

${ }^{3} E \mathrm{P} A$, , 1993 Diesel Desulfurization Question and Answer Document," p. 1.
} 
Figure 1 illustrates how the colors of diesel fuels and blending stocks will change on October 1 . Of course there will undoubtedly be many degrees and shades of colors between each of these descrihed since (1) initial color of fuel varies depending on the chemical make up of the crude oil; ( 2 ) no particular end color is required; and (3) no specific amount of dye is required by EPA.

Figure 1. Changes in the Color of Diesel Fuel on October 1, 1993

\begin{tabular}{|c|c|c|c|}
\hline \multirow{3}{*}{ Fuel } & \multicolumn{3}{|c|}{ Colors } \\
\hline & Early 1993 & \multicolumn{2}{|c|}{$\begin{array}{l}\text { October 1, } 1993 \\
\text { EPA Roquirements }\end{array}$} \\
\hline & Low/High Sulfur & Low Sulfur & High Sulfur \\
\hline Kerosene & Clear & Clear & \\
\hline Kero-jet & Clear & Clear & $\begin{array}{l}\text { Clear: aircraft use } \\
\text { Blue: other uses }\end{array}$ \\
\hline No. 1 Distllat & Clear & Clear, Blue ${ }^{a}$ & Blue \\
\hline No. 2 Distillato & $\begin{array}{l}\text { Clear, Straw: natural } \\
\text { Green, Red, Purple: premium } \\
\text { Red: oft-highway diesel from Canada }\end{array}$ & $\begin{array}{l}\text { Clear, Straw: natural } \\
\text { Red, Purple: premium }\end{array}$ & $\begin{array}{l}\text { Blue, Green, Coffee: dyed } \\
\text { Clear, Straw: DOD marine diesel }\end{array}$ \\
\hline
\end{tabular}

${ }^{a}$ May be dyed blue "s intended for oft-highway uses only or mixed with some high sulfur No. 1 distillate

\section{Definition of "Low Sulfur"}

Congress (in the Clean Air Act Amendments of 1990) anc EPA (in its final rule) defined low sulfur as a maximum content of 0.05 percent sulfur by weight. An enforcement issue arose concerning sulfur levels between 0.05 and 0.06 percent by weight. EPA's position is that "as a matter of enforcement discretion," it "will take enforcement action only when its diesel sulfur test results are 0.055 weight percent or greater "14
Pipeline companies are requiring "on-highway" diesel fuel to meet more stringent specifications than EPA. They want to ensure that, even if a small amount of high sulfur fuel sontaminated a low sulfur batch, the low sulfur batch would remain in compliance with EPA regulations. Colonial and Williams pipelines, for example, are requiring low sulfur on-highway diesel to contain no more than 0.047 percent sulfur content by weight. ${ }^{5}$ Oil companies that want to ship on-highway diesel fuel by pipeline will have to meet the specifications required by the pipeline (as well as EPA) which means that the diesel fuel produced and shipped will be below EPA's enforcement threshold of 0.055 percent sulfur content by weight.

\section{California's Clean Diesel Regulations}

Effective October 1, 1993, on- and off-highway motor vehicle diesel fuel sold in California will be limited to a maximum of 0.05 percent sulfur content by weight and must have aromatics reduced to no more than 10 percent volume, unless a refiner can prove that an alternative formulation will reduce emissions by an equivalent amount. The low sulfur regulations issued by the California Air Resources Board apply to such off-highway uses as agriculture, construction, and mining, but excludes stationary, marine, and railroad uses. The South Coast Air Basin (greater Los Angeles area) and Ventura county impose even stricter standards, requiring all No. 2 distillate sold in the area be both low sulfur and low aromatics.

\footnotetext{
"Environmental Protection Agency (EPA), "1993 Diesel Desulfurization Question and Answer Document," pp, 8.9; El'A also stated that it "will take enforcement action only as a result of a calculated cetane index of less tha 39.5 ."

${ }^{5}$ Colonial also requires a higher minimum cetane rating than El'A. Colonial requires it to be 42 while El'A only requires it to be 40 (with enforcement occurring if below 39.5).
} 


\section{Impact of Requirements on Petroleum Marketing}

No. 2 diesel is not the only petroleum product affected by the low sulfur requirements. During winter months, kerosene, No. 1 distillate, or kero-jet is typ $\mathrm{i}$ cally blended into diesel fuel to prevent clogging of fu:l lines. No. 1 distillate and kero-jet are not normally low sulfur products. The supply of low sulfur No. 1 distillate and kero-jet will probably increase as a result of the regulations. The demand for "sweet" or low sulfur crude oil may increase as refiners try to position themselves to meet the new requirements for low sulfur diesel.

Storage and distribution of all petroleum products will become more challenging for pipelines, terminals, distributors, and retailers. Some pipeline companies, like Williams, for example, will no longer ship high sulfur No. 1 distillate. Some oil companies, particularly the smaller firms, will only sell high or low sulfur diesel. Even large oil companies that will market all products have announced that at selected terminals they will only carry high or low sulfur No. 1 and No. 2 distillate. Options open to companies to retain both on-highway and off-highway sales in a particular area include: (1) manage available storage capacity to accommodate both low and high sulfur products; (2) enter into exchange agreements with competitors to obtain products not stored; (3) carry low sulfur products only and market these to all customers; and (4) buy or lease additional storage capacity.

\section{IRS Requirements: Exempt-use Diesel will be Dyed}

Section 13242 of the Omnibus Budget Reconciliation Act of 1993 revises the Federal diesel fuel tax. Effective January 1, 1994, the tax collection point for diesel fuel is moved to the terminal. The tax must be paid unless the diesel fuel is destined for a nontaxable use and the fuel is indelibly dyed. Clear low sulfur fuel (which is taxed) that is used for tax exempt purposes will entitle the user to claim a refund. Any diesel fuel that is dyed will not be taxed and, hence, will not be allowed for use on-highway. Discussions between IRS and EPA and between IRS and oil company officials are ongoing and comments are being solicited from the public.

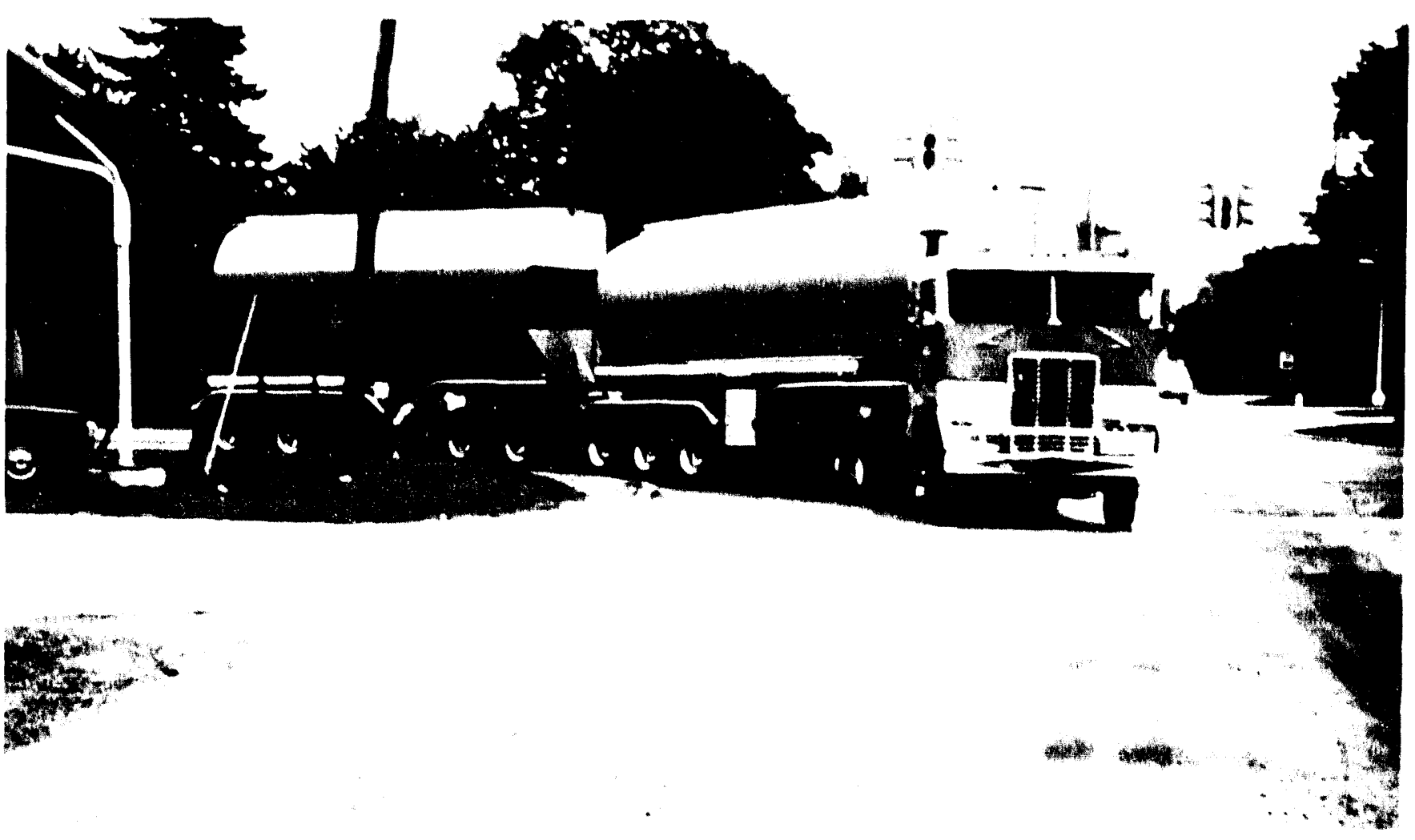

All on-highway diesel must be low sulfur (.05 percent by weight) effective October 1, 1993. 
Table 1. Sales of Distlllate Fuel Oll by End Use In the United States: 1988-1992 (Thousand Gallons)

\begin{tabular}{|c|c|c|c|c|c|}
\hline \multirow{2}{*}{ End Use } & \multicolumn{5}{|c|}{ Distlliate Fuel Oil } \\
\hline & 1988 & 1989 & 1990 & 1991 & 1992 \\
\hline U.S. Total & $47,876,811$ & $49,788,896$ & $47,826,587$ & $46,210,737$ & $47,281,652$ \\
\hline 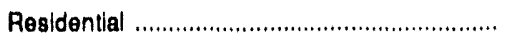 & $8,518,859$ & $8,648,629$ & $7,277,365$ & $6,775,589$ & $7,291,444$ \\
\hline 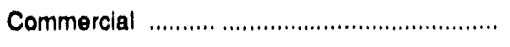 & $4,294,182$ & $4,277,105$ & $3,986,624$ & $3,767,833$ & $3,771,021$ \\
\hline Industrial & $2,562,685$ & $2,725,774$ & $2,593,312$ & $2,309,184$ & $2,312,146$ \\
\hline 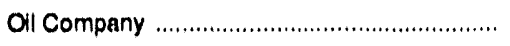 & 622,736 & 683,683 & 751,450 & 738,618 & 664,408 \\
\hline Farm …….... & $3,162,575$ & $3,360,092$ & $3,403,400$ & $3,158,477$ & $3,489,518$ \\
\hline 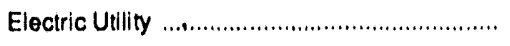 & 720,137 & 887,599 & 763,709 & 594,821 & 540,683 \\
\hline Rallroad & $3,095,736$ & $3,240,648$ & $3,104,630$ & $2,879,109$ & $3,172,724$ \\
\hline 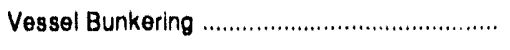 & $1,991,773$ & $2,258,465$ & $2,064,842$ & $2,046,064$ & $2,218,592$ \\
\hline 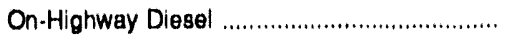 & $20,038,691$ & $21,131,692$ & $21,360,477$ & $20,485,364$ & $21,374,872$ \\
\hline 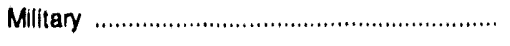 & 830,614 & 852,838 & 709,232 & 811,705 & 652,507 \\
\hline 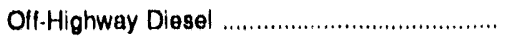 & $1,671,387$ & $1,689,651$ & $1,808,646$ & $1,641,560$ & $1,757,788$ \\
\hline 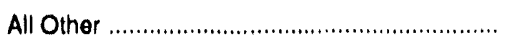 & 67,435 & 32,719 & 2,898 & 2,413 & 5,848 \\
\hline
\end{tabular}

Note: Totals may not equal sum of components due to independent rounding.

Sources: - Energy Intormation Administration Form ElA-821, "Annual Fuel Oil and Kerosene Sales Report," for $1988-1992$.

- On-Highway Diesel data are Federal Highway Administation statistics of highway special fuels use.

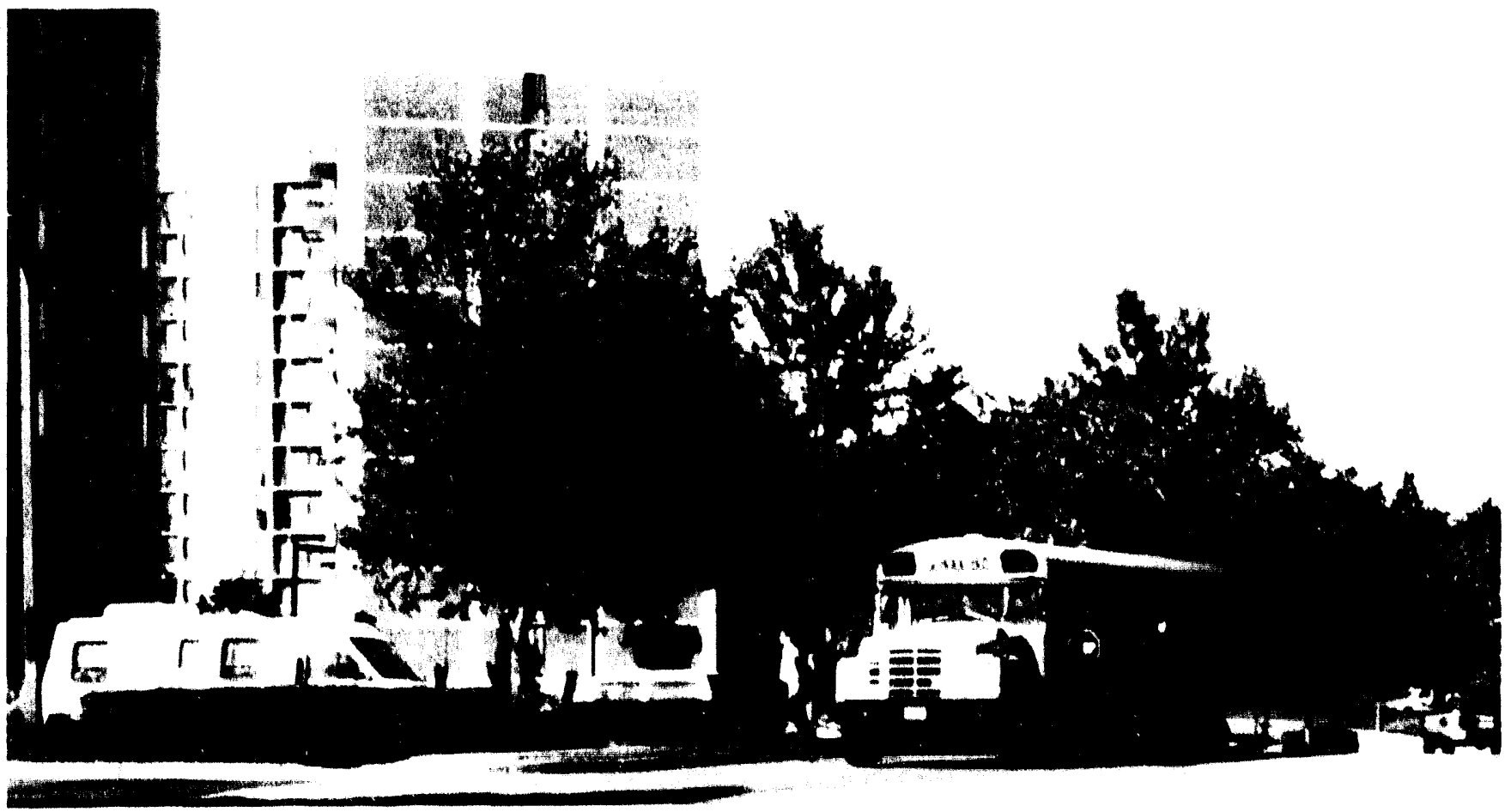

Sales to school buses and apartment buildings are considered commercial use. 
Table 2. Sales of Residual Fuel Oll by End Use In the United States: 1988-1992 (Thousand Gallons)

\begin{tabular}{|c|c|c|c|c|c|}
\hline \multirow{2}{*}{ End Uae } & \multicolumn{5}{|c|}{ Resldual Fuel Oll } \\
\hline & 1980 & 1989 & 1990 & 1991 & 1992 \\
\hline U.S. Total & $21,719,226$ & $21,829,300$ & $19,233,428$ & $17,631,823$ & $16,199,042$ \\
\hline 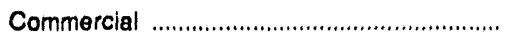 & $1,927,533$ & $1,792,634$ & $1,522,125$ & $1,423,948$ & $1,176,048$ \\
\hline 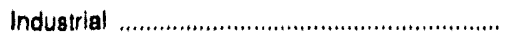 & $3,239,538$ & $2,665,438$ & $2,255,759$ & $1,934,793$ & $2,126,857$ \\
\hline 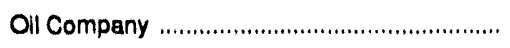 & 548,930 & 374,469 & 318,298 & 300,939 & 271,070 \\
\hline Flectric Utillty & $0,464,467$ & $9,680,974$ & $8,679,851$ & $7,071,254$ & $5,961,441$ \\
\hline 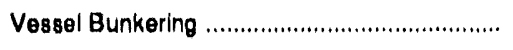 & $6,354,536$ & $7,191,689$ & $6,326,322$ & $6,773,174$ & $6,563,220$ \\
\hline 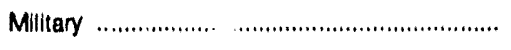 & 125,318 & 92,229 & 101,821 & 118,976 & 91,791 \\
\hline 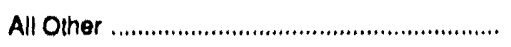 & 58,812 & 31,877 & 29,152 & 7,738 & 8,613 \\
\hline
\end{tabular}

Note: Totals may not equal sum of components due to independent rounding.

Sources: - Energy Information Administration Form ElA-821, "Annual Fuel Oil and Kerosene Sales Report," for 1988.1992.

Table 3. Sales of Kerosene by End Use In the United States: 1988-1992 (Thousand Gallons)

\begin{tabular}{|c|c|c|c|c|c|}
\hline \multirow{2}{*}{ End Uae } & \multicolumn{5}{|c|}{ Korosene } \\
\hline & 1988 & 1989 & 1980 & 1891 & 1092 \\
\hline 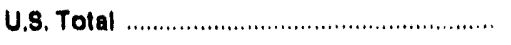 & $1,478,316$ & $1,291,668$ & 652,008 & 708,716 & 636,426 \\
\hline 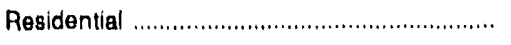 & $1,063,187$ & 868.728 & 473,409 & 535,864 & 481,511 \\
\hline 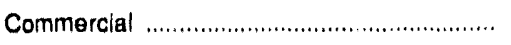 & 193,476 & 204.258 & 87.672 & 89,542 & 82,398 \\
\hline 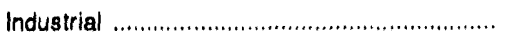 & 117,858 & 124,003 & 60,432 & 52,438 & 46,099 \\
\hline 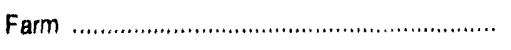 & 33,426 & 30,081 & 16,691 & 17,053 & 20,036 \\
\hline 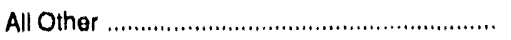 & 70,370 & 64,598 & 13,804 & 14,819 & 6,382 \\
\hline
\end{tabular}

Note: Totals may not equal sum of components due to independent rounding.

Note: Kerosene data in the Sales tables $(1-12)$ are adjusted at the national level. See Technical Note 3 for further explanation.

Sources: - Kerosene data are based on data from the Energy Intormation Administration's "Pelroleum Supply Annual," Volume 1, 1988 . 1992, (DOE/EIA-0340(88)/1 - (92)/1). 
Table 4. Sales of Dlstillate Fuel Oll by End Use, 1991 and 1992 (Thousand Galions)

\begin{tabular}{|c|c|c|c|c|c|c|}
\hline \multirow{2}{*}{ Dee'ination } & \multicolumn{2}{|c|}{ Reoldentlal } & \multicolumn{2}{|c|}{ Commerclal } & \multicolumn{2}{|c|}{ Industrlal } \\
\hline & 1991 & 1002 & 1091 & 1992 & 1891 & 1002 \\
\hline 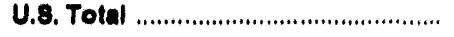 & $6,778,680$ & $7,291,444$ & $3,767,633$ & $3,771,021$ & $2,309,184$ & $2,312,146$ \\
\hline 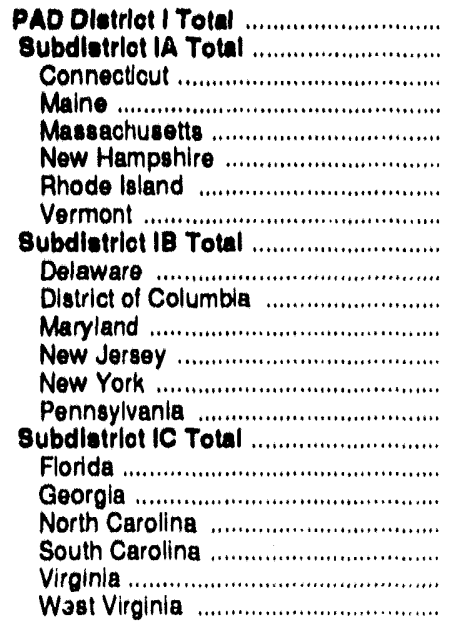 & $\begin{array}{r}5,401,686 \\
2,029,118 \\
551,718 \\
253,239 \\
817,102 \\
175,089 \\
131,088 \\
99,984 \\
2,893,084 \\
48,927 \\
8,106 \\
205,297 \\
543,537 \\
1,228,670 \\
858,448 \\
478,583 \\
11,658 \\
8,764 \\
157,197 \\
49,010 \\
225,534 \\
26,389\end{array}$ & $\begin{array}{r}6,003,780 \\
2,362,734 \\
677,218 \\
266,252 \\
948,330 \\
185,640 \\
164,860 \\
110,434 \\
3,180,024 \\
52,484 \\
8,563 \\
224,705 \\
564,623 \\
1,411,324 \\
889,225 \\
600,122 \\
15,594 \\
8,950 \\
176,502 \\
34,767 \\
241,017 \\
23,282\end{array}$ & $\begin{array}{r}2,633,817 \\
719,806 \\
146,539 \\
70,814 \\
373,687 \\
55,978 \\
38,076 \\
34,371 \\
1,379,380 \\
21,601 \\
28,833 \\
112,806 \\
322,065 \\
626,457 \\
267,618 \\
434,871 \\
147,334 \\
42,347 \\
89,432 \\
25,659 \\
104,709 \\
25,390\end{array}$ & $\begin{array}{r}2,604,432 \\
700,243 \\
148,381 \\
86,466 \\
336,992 \\
56,911 \\
30,374 \\
41,119 \\
1,460,289 \\
17,577 \\
27,772 \\
129,816 \\
320,797 \\
700,658 \\
272,669 \\
434,899 \\
151,354 \\
52,325 \\
82,645 \\
33,825 \\
98,540 \\
16,210\end{array}$ & $\begin{array}{r}730,243 \\
111,084 \\
32,901 \\
21,732 \\
31,770 \\
9,025 \\
7,254 \\
8,382 \\
261,086 \\
8,147 \\
11 \\
30,618 \\
61,028 \\
53,251 \\
128,126 \\
388,093 \\
38,931 \\
53,631 \\
68,487 \\
32,411 \\
69,865 \\
94,667\end{array}$ & $\begin{array}{r}781,208 \\
186,408 \\
29,431 \\
23,476 \\
70,094 \\
14,148 \\
10,461 \\
8,795 \\
282,950 \\
7,536 \\
96 \\
24,615 \\
59,727 \\
47,985 \\
142,992 \\
341,863 \\
49,745 \\
31,181 \\
80,200 \\
39,116 \\
49,562 \\
92,069\end{array}$ \\
\hline 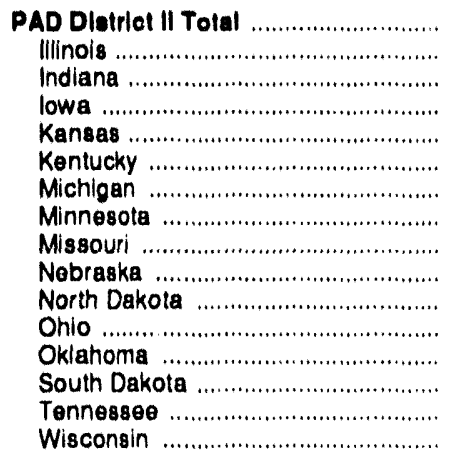 & $\begin{array}{r}1,074,198 \\
51,948 \\
81,988 \\
37,525 \\
990 \\
29,758 \\
192,877 \\
173,405 \\
18,191 \\
8,353 \\
38,170 \\
178,617 \\
3 \\
34,008 \\
11,357 \\
217,006\end{array}$ & $\begin{array}{r}1,007,648 \\
42,976 \\
81,601 \\
33,480 \\
1,240 \\
33,067 \\
182,056 \\
147,363 \\
15,386 \\
6,251 \\
27,609 \\
200,534 \\
85 \\
20,382 \\
11,150 \\
204,448\end{array}$ & $\begin{array}{r}\mathbf{5 7 8 , 1 8 7} \\
71,493 \\
49,773 \\
2,3,839 \\
15,355 \\
30,283 \\
82,018 \\
38,515 \\
47,017 \\
7,743 \\
6,758 \\
68,353 \\
20,529 \\
8,108 \\
25,472 \\
82,932\end{array}$ & $\begin{array}{r}606,887 \\
77,489 \\
60,883 \\
20,996 \\
21,576 \\
37,771 \\
76,025 \\
32,674 \\
50,517 \\
11,604 \\
6,770 \\
72,390 \\
16,100 \\
10,555 \\
44,807 \\
66,728\end{array}$ & $\begin{array}{r}878,713 \\
83,494 \\
92,580 \\
16,070 \\
7,935 \\
154,816 \\
53,275 \\
38,726 \\
28,208 \\
4,228 \\
17,819 \\
99,890 \\
24,889 \\
5,390 \\
32,763 \\
18,529\end{array}$ & $\begin{array}{r}731,018 \\
101,868 \\
101,157 \\
17,267 \\
9,731 \\
160,579 \\
52,358 \\
36,453 \\
27,356 \\
6,630 \\
10,357 \\
124,528 \\
19,151 \\
10,653 \\
35,018 \\
17,914\end{array}$ \\
\hline 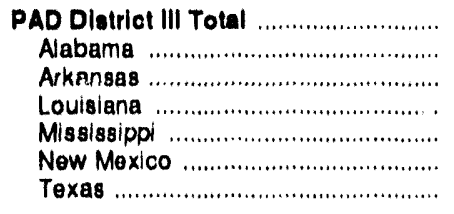 & $\begin{array}{r}1,098 \\
580 \\
25 \\
60 \\
56 \\
273 \\
105\end{array}$ & $\begin{array}{r}1,348 \\
349 \\
410 \\
2 \\
42 \\
468 \\
74\end{array}$ & $\begin{array}{r}199,549 \\
31,406 \\
10,931 \\
28,724 \\
19,417 \\
14,772 \\
94,299\end{array}$ & $\begin{array}{r}191,736 \\
33,653 \\
12,343 \\
19,788 \\
16,683 \\
7,861 \\
101,406\end{array}$ & $\begin{array}{r}280,698 \\
86,858 \\
12,187 \\
22,361 \\
15,001 \\
25,962 \\
118,326\end{array}$ & $\begin{array}{r}277,079 \\
74,830 \\
11,367 \\
38,666 \\
24,007 \\
13,199 \\
115,009\end{array}$ \\
\hline 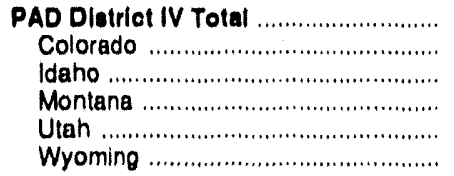 & $\begin{array}{r}46,628 \\
921 \\
24,051 \\
12,168 \\
5,517 \\
2,971\end{array}$ & $\begin{array}{r}36,233 \\
772 \\
20,429 \\
7,802 \\
4,135 \\
2,096\end{array}$ & $\begin{array}{r}66,238 \\
20,191 \\
14,828 \\
6,958 \\
16,042 \\
8,217\end{array}$ & $\begin{array}{r}76,602 \\
29,892 \\
14,854 \\
6,066 \\
16,842 \\
7,947\end{array}$ & $\begin{array}{r}200,167 \\
38,592 \\
32,062 \\
46,479 \\
34,505 \\
48,529\end{array}$ & $\begin{array}{r}164,694 \\
43,586 \\
11,565 \\
34,000 \\
28,450 \\
47,085\end{array}$ \\
\hline 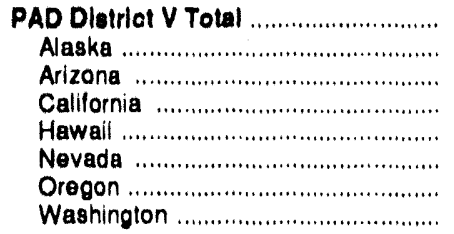 & $\begin{array}{r}283,009 \\
67,421 \\
214 \\
8,407 \\
16 \\
9,344 \\
62,806 \\
104,802\end{array}$ & $\begin{array}{r}243,438 \\
79,380 \\
258 \\
9,914 \\
14 \\
10,718 \\
52,806 \\
90,347\end{array}$ & $\begin{array}{r}390,044 \\
41,141 \\
12,799 \\
187,842 \\
25,867 \\
12,399 \\
41,892 \\
68,004\end{array}$ & $\begin{array}{r}292,365 \\
68,050 \\
11,159 \\
98,593 \\
21,594 \\
14,707 \\
37,929 \\
40,332\end{array}$ & $\begin{array}{r}399,368 \\
10,331 \\
54,476 \\
170,882 \\
4,507 \\
103,802 \\
19,648 \\
35,719\end{array}$ & $\begin{array}{r}368,147 \\
20,887 \\
72,538 \\
68,474 \\
6,268 \\
121,422 \\
31,067 \\
37,492\end{array}$ \\
\hline
\end{tabular}

See footnotes at end of table. 
Table 4. Sales of Distlliate Fuel Oll by End Use, 1991 and 1992 (Continued) (Thousand Gallons)

\begin{tabular}{|c|c|c|c|c|c|c|}
\hline \multirow{2}{*}{ Destination } & \multicolumn{2}{|c|}{ Oll Company } & \multicolumn{2}{|c|}{ Farm } & \multicolumn{2}{|c|}{ Elootrlo Utillty } \\
\hline & 1991 & 1992 & 1991 & 1992 & 1981 & 1992 \\
\hline 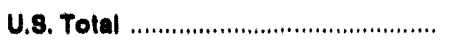 & 738,618 & 664,408 & $3,158,477$ & $3,409,618$ & 804,821 & 640,683 \\
\hline 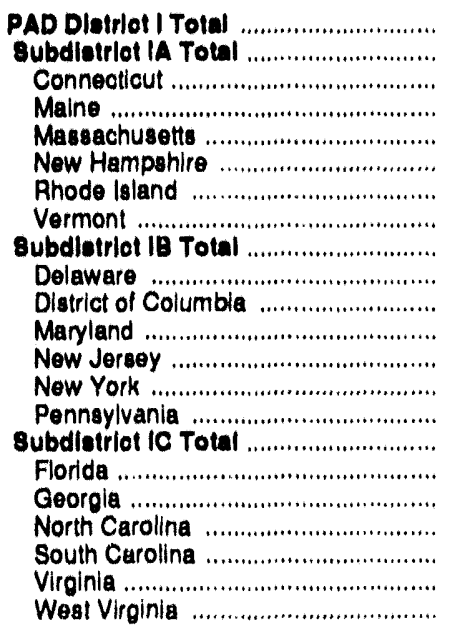 & $\begin{array}{r}23,613 \\
4,891 \\
468 \\
3,323 \\
463 \\
63 \\
285 \\
20 \\
12,298 \\
342 \\
-9 \\
893 \\
2,727 \\
804 \\
7,529 \\
6,726 \\
1,407 \\
437 \\
114 \\
480 \\
815 \\
3,503\end{array}$ & $\begin{array}{r}16,708 \\
1,316 \\
403 \\
115 \\
413 \\
71 \\
291 \\
22 \\
9,679 \\
343 \\
-09 \\
781 \\
3,085 \\
628 \\
4,743 \\
8,873 \\
1,828 \\
291 \\
145 \\
194 \\
1,050 \\
2,366\end{array}$ & $\begin{array}{r}439,808 \\
29,346 \\
2,602 \\
5,002 \\
6,354 \\
4,547 \\
1,336 \\
8,504 \\
158,839 \\
8,638 \\
21,657 \\
22,685 \\
51,073 \\
51,877 \\
254,624 \\
55,587 \\
62,537 \\
44,997 \\
46,143 \\
38,750 \\
6,611\end{array}$ & $\begin{array}{r}440,178 \\
27,884 \\
5,675 \\
4,143 \\
4,760 \\
3,003 \\
641 \\
9,863 \\
186,326 \\
5,867 \\
- \\
22,815 \\
9,442 \\
57,650 \\
60,250 \\
285,868 \\
65,230 \\
55,657 \\
50,898 \\
28,816 \\
47,997 \\
6,370\end{array}$ & $\begin{array}{r}288,861 \\
28,898 \\
5,120 \\
847 \\
7,777 \\
10,587 \\
1,138 \\
418 \\
122,001 \\
4,085 \\
36,765 \\
7,564 \\
9,826 \\
19,892 \\
44,079 \\
117,962 \\
61,873 \\
6,640 \\
13,191 \\
6,671 \\
14,626 \\
15,261\end{array}$ & $\begin{array}{r}239,921 \\
18,749 \\
2,965 \\
806 \\
11,826 \\
1,150 \\
1,814 \\
388 \\
88,162 \\
4,366 \\
20,315 \\
11,027 \\
10,288 \\
11,431 \\
29,846 \\
133,020 \\
75,601 \\
10,834 \\
13,776 \\
6,131 \\
11,780 \\
14,897\end{array}$ \\
\hline 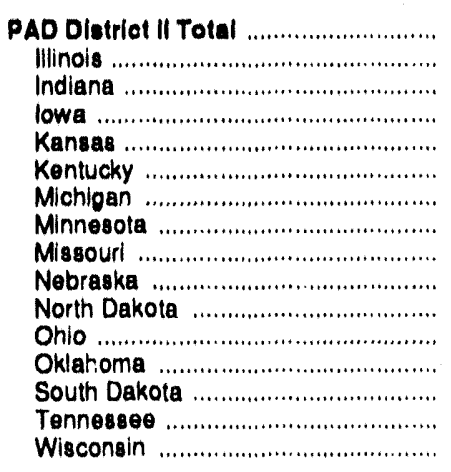 & $\begin{array}{r}63,844 \\
1,232 \\
1,293 \\
147 \\
11,744 \\
1,082 \\
1,376 \\
734 \\
414 \\
70 \\
5,807 \\
3,727 \\
34,842 \\
19 \\
695 \\
641\end{array}$ & $\begin{array}{r}83,440 \\
1,720 \\
1,361 \\
156 \\
4,846 \\
2,577 \\
6,489 \\
878 \\
369 \\
29 \\
5,793 \\
5,027 \\
52,488 \\
19 \\
1,081 \\
608\end{array}$ & $\begin{array}{r}1,802,181 \\
181,287 \\
103,471 \\
166,960 \\
157,317 \\
40,060 \\
79,952 \\
156,791 \\
64,811 \\
181,956 \\
100,834 \\
81,234 \\
44,547 \\
87,755 \\
45,585 \\
89,502\end{array}$ & $\begin{array}{r}1,840,728 \\
207,698 \\
90,400 \\
225,190 \\
163,248 \\
55,733 \\
98,024 \\
193,001 \\
74,465 \\
194,423 \\
107,954 \\
94,530 \\
89,645 \\
74,701 \\
48,291 \\
123,334\end{array}$ & $\begin{array}{r}143,301 \\
21,623 \\
15,282 \\
2,100 \\
4,811 \\
12,040 \\
20,261 \\
4,800 \\
9,479 \\
1,064 \\
2,244 \\
30,367 \\
431 \\
724 \\
7,352 \\
10,833\end{array}$ & $\begin{array}{r}128,049 \\
19,636 \\
12,972 \\
3,312 \\
3,677 \\
13,363 \\
27,924 \\
4,091 \\
4,514 \\
674 \\
2,046 \\
18,186 \\
1,422 \\
2 \\
10,200 \\
3,130\end{array}$ \\
\hline 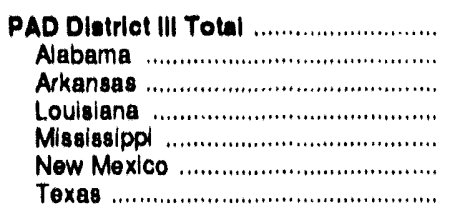 & $\begin{array}{r}848,741 \\
10,859 \\
2,817 \\
270,421 \\
5,219 \\
11,495 \\
247,830\end{array}$ & $\begin{array}{r}484,664 \\
8,439 \\
2,576 \\
230,261 \\
8,948 \\
10,601 \\
183,729\end{array}$ & $\begin{array}{r}600,926 \\
34,228 \\
51,514 \\
68,185 \\
114,997 \\
13,239 \\
227,761\end{array}$ & $\begin{array}{r}846,898 \\
37,717 \\
86,011 \\
58,839 \\
80,845 \\
21,400 \\
260,876\end{array}$ & $\begin{array}{r}46,022 \\
9,059 \\
8,495 \\
3,853 \\
3,692 \\
6,143 \\
15,780\end{array}$ & $\begin{array}{r}40,481 \\
8,643 \\
7,774 \\
4,885 \\
1,154 \\
826 \\
17,180\end{array}$ \\
\hline 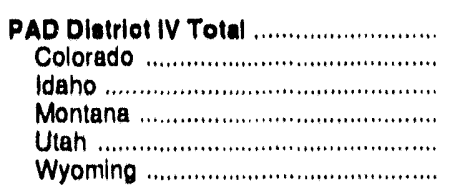 & $\begin{array}{r}19,007 \\
3,097 \\
61 \\
2,909 \\
3,650 \\
10,190\end{array}$ & $\begin{array}{r}37,122 \\
8,270 \\
53 \\
910 \\
8,689 \\
18,200\end{array}$ & $\begin{array}{r}201,136 \\
44,376 \\
75,383 \\
58,483 \\
10,293 \\
12,602\end{array}$ & $\begin{array}{r}204,607 \\
72,453 \\
63,207 \\
39,494 \\
14,963 \\
14,490\end{array}$ & $\begin{array}{r}9,924 \\
1,731 \\
465 \\
1,064 \\
2,335 \\
4,330\end{array}$ & $\begin{array}{r}6,483 \\
1,570 \\
58 \\
158 \\
1,676 \\
3,020\end{array}$ \\
\hline 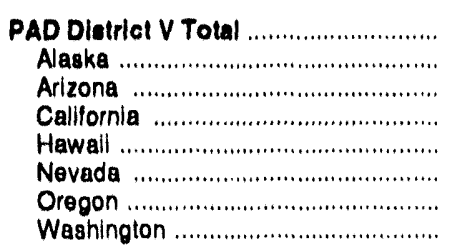 & $\begin{array}{r}82,414 \\
59,159 \\
309 \\
11,198 \\
1,176 \\
239 \\
269 \\
10,064\end{array}$ & $\begin{array}{r}72,524 \\
56,323 \\
203 \\
14,412 \\
1,227 \\
222 \\
4 \\
132\end{array}$ & $\begin{array}{r}416,447 \\
- \\
17,389 \\
271,422 \\
9,689 \\
5,586 \\
42,521 \\
68,630\end{array}$ & $\begin{array}{r}467,108 \\
1 \\
19,819 \\
318,799 \\
11,489 \\
5,378 \\
42,851 \\
68,771\end{array}$ & $\begin{array}{r}129,713 \\
25,763 \\
2,811 \\
11,610 \\
75,134 \\
5,442 \\
3,607 \\
5,346\end{array}$ & $\begin{array}{r}128,770 \\
36,452 \\
3,183 \\
6,889 \\
79,953 \\
539 \\
1,207 \\
538\end{array}$ \\
\hline
\end{tabular}

See footnotes at end of table. 
Table 4. Sales of Distillate Fuel Oll by End Use, 1991 and 1992 (Continued) (Thousand Gallons)

\begin{tabular}{|c|c|c|c|c|c|c|}
\hline \multirow{2}{*}{ Dostlnatlon } & \multicolumn{2}{|c|}{ Rallioad } & \multicolumn{2}{|c|}{ Veseel Bunkering } & \multicolumn{2}{|c|}{ On-Hlohway } \\
\hline & 1901 & 1992 & 1001 & 1092 & 1001 & 1002 \\
\hline 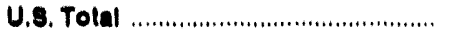 & $2,879,109$ & $3,172,724$ & $2,046,064$ & $2,218,802$ & $20,486,364$ & $21,374,872$ \\
\hline 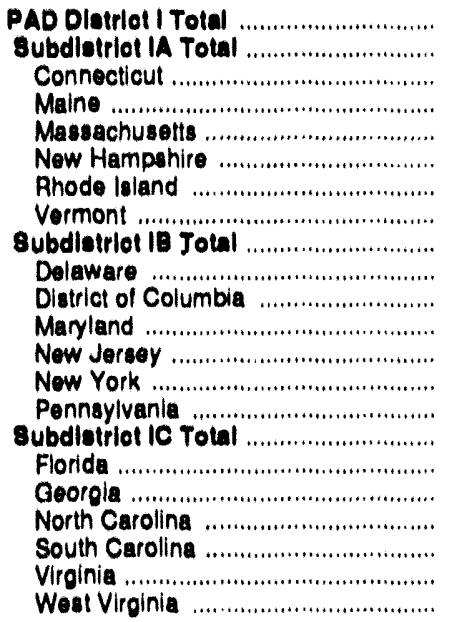 & 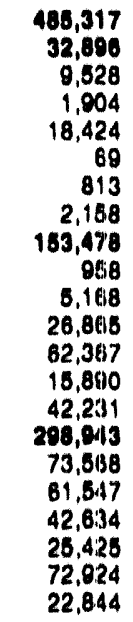 & $\begin{array}{r}801,734 \\
32,807 \\
10,952 \\
4,628 \\
12,813 \\
610 \\
842 \\
2,820 \\
248,384 \\
1,303 \\
6,346 \\
29,497 \\
124,174 \\
35,069 \\
48,964 \\
323,613 \\
89,989 \\
65,351 \\
48,648 \\
18,008 \\
81,475 \\
22,372\end{array}$ & $\begin{array}{r}481,024 \\
76,087 \\
2,383 \\
15,986 \\
48,199 \\
181 \\
8,921 \\
408 \\
164,211 \\
6,944 \\
6 \\
9,075 \\
93,082 \\
13,741 \\
31,362 \\
281,328 \\
116,630 \\
22,336 \\
14,972 \\
5,437 \\
40,966 \\
50,976\end{array}$ & $\begin{array}{r}886,892 \\
88,610 \\
5,687 \\
13,847 \\
59,661 \\
370 \\
8,561 \\
383 \\
161,017 \\
3,916 \\
7 \\
11,887 \\
94,662 \\
19,676 \\
30,879 \\
307,386 \\
165,609 \\
35,278 \\
10,744 \\
8,453 \\
41,873 \\
55,288\end{array}$ & $\begin{array}{r}8, \times 06,989 \\
864,206 \\
173,819 \\
105,847 \\
248,685 \\
48,348 \\
39,759 \\
41,078 \\
2,811,811 \\
52,101 \\
21,800 \\
327,298 \\
389,054 \\
786,976 \\
934,185 \\
3,341,272 \\
806,168 \\
854,684 \\
598,207 \\
360,461 \\
547,591 \\
174,151\end{array}$ & $\begin{array}{r}8,886,163 \\
893,219 \\
185,287 \\
113,196 \\
247,409 \\
50,760 \\
37,344 \\
59,223 \\
2,669,827 \\
51,978 \\
20,881 \\
341,612 \\
373,082 \\
800,229 \\
881,846 \\
3,628,317 \\
893,762 \\
874,159 \\
661,167 \\
418,909 \\
586,726 \\
193,594\end{array}$ \\
\hline 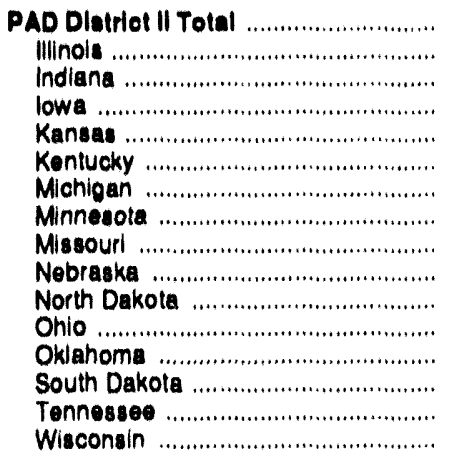 & $\begin{array}{r}1,189,078 \\
120,118 \\
193,917 \\
29,868 \\
187,708 \\
101,029 \\
36,099 \\
68,211 \\
24,4118 \\
112,4188 \\
39,764 \\
96,868 \\
85,830 \\
4,346 \\
71,537 \\
37,010\end{array}$ & $\begin{array}{r}1,242,347 \\
117,126 \\
192,713 \\
34,501 \\
146,012 \\
97,874 \\
32,827 \\
117,065 \\
16,294 \\
126,990 \\
38,901 \\
122,684 \\
101,745 \\
4,378 \\
71,814 \\
22,722\end{array}$ & $\begin{array}{r}416,179 \\
54,882 \\
9,817 \\
4,617 \\
- \\
139,616 \\
4,503 \\
3,303 \\
40,054 \\
- \\
- \\
14,645 \\
- \\
- \\
140,703 \\
3,078\end{array}$ & $\begin{array}{r}440,404 \\
58,141 \\
13,578 \\
4,362 \\
- \\
139,600 \\
9,288 \\
4,870 \\
69,442 \\
- \\
- \\
14,387 \\
252 \\
- \\
123,277 \\
3,009\end{array}$ & $\begin{array}{r}6,838,180 \\
877,536 \\
784,039 \\
319,894 \\
276,312 \\
417,619 \\
531,309 \\
369,176 \\
584,823 \\
220,319 \\
94,596 \\
886,816 \\
352,086 \\
99,489 \\
575,503 \\
448,663\end{array}$ & $\begin{array}{r}7,093,042 \\
865,402 \\
783,113 \\
324,321 \\
258,207 \\
513,264 \\
566,807 \\
350,224 \\
644,586 \\
242,727 \\
97,448 \\
913,607 \\
390,810 \\
102,874 \\
586,009 \\
484,846\end{array}$ \\
\hline 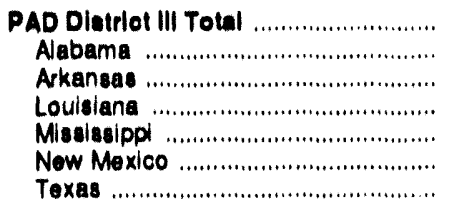 & $\begin{array}{r}847,040 \\
56,005 \\
35,047 \\
54,595 \\
20,193 \\
40,074 \\
340,227\end{array}$ & $\begin{array}{r}867,178 \\
65,488 \\
28,607 \\
36,293 \\
24,632 \\
74,629 \\
448,780\end{array}$ & $\begin{array}{r}830,187 \\
76,318 \\
1,898 \\
323,830 \\
51,801 \\
177,239\end{array}$ & $\begin{array}{r}706,186 \\
73,189 \\
1,782 \\
323,747 \\
54,647 \\
252,789\end{array}$ & $\begin{array}{r}3,390,672 \\
548,437 \\
384,746 \\
366,785 \\
316,687 \\
219,884 \\
1,554,483\end{array}$ & $\begin{array}{r}3,445,063 \\
578,189 \\
389,095 \\
348,020 \\
317,128 \\
228,791 \\
1,575,936\end{array}$ \\
\hline 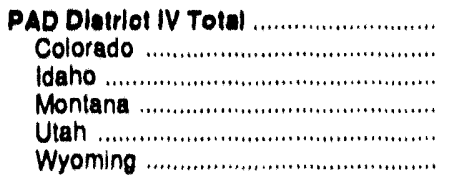 & $\begin{array}{l}281,601 \\
77,880 \\
21,382 \\
48,427 \\
36,677 \\
67,137\end{array}$ & $\begin{array}{r}264,007 \\
66,656 \\
20,093 \\
54,429 \\
40,704 \\
73,215\end{array}$ & $\begin{array}{r}167 \\
- \\
167 \\
- \\
-\end{array}$ & $\begin{array}{r}112 \\
\overline{82} \\
\overline{20} \\
-\end{array}$ & $\begin{array}{l}782,786 \\
216,076 \\
124,668 \\
114,876 \\
170.272 \\
156,894\end{array}$ & $\begin{array}{l}828,826 \\
215,220 \\
128,768 \\
132,587 \\
180,007 \\
172,244\end{array}$ \\
\hline 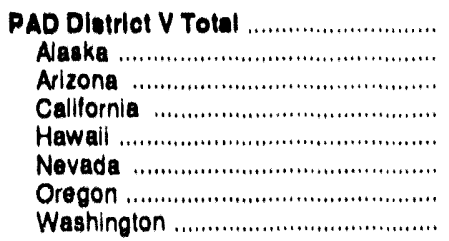 & $\begin{array}{r}408,272 \\
4,730 \\
4,365 \\
238,832 \\
12 \\
6,008 \\
88,845 \\
62,480\end{array}$ & $\begin{array}{r}408,488 \\
5,296 \\
7,120 \\
217,250 \\
22 \\
4,164 \\
114,668 \\
57,947\end{array}$ & $\begin{array}{r}518,907 \\
138,606 \\
170,763 \\
87,333 \\
- \\
54,608 \\
67,698\end{array}$ & $\begin{array}{r}814,938 \\
153,214 \\
23 \\
111,884 \\
89,687 \\
- \\
50,248 \\
109,811\end{array}$ & $\begin{array}{r}2,966,737 \\
39,627 \\
300,734 \\
1,769,575 \\
25,364 \\
140,148 \\
333,566 \\
357,723\end{array}$ & $\begin{array}{r}3,118,678 \\
49,225 \\
351,627 \\
1,842,723 \\
24,850 \\
152,457 \\
329,762 \\
368,334\end{array}$ \\
\hline
\end{tabular}

See lootnotes at end of table. 
Table 4. Sales of Distillate Fuel Oll by End Use, 1991 and 1992 (Continued) (Thousand Gallons)

\begin{tabular}{|c|c|c|c|c|c|c|c|c|}
\hline \multirow{2}{*}{ Desefination } & \multicolumn{2}{|c|}{ Milltary } & \multicolumn{2}{|c|}{ Off-HIghway } & \multicolumn{2}{|c|}{ All Othor } & \multicolumn{2}{|c|}{ Total } \\
\hline & 1991 & 1002 & 1991 & 1902 & 1991 & 1992 & 1001 & 1992 \\
\hline 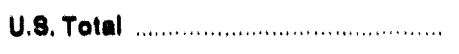 & 811,708 & 632,507 & $1,641,560$ & $1,767,788$ & 2,413 & 5,848 & $48,210,737$ & $47,261,532$ \\
\hline 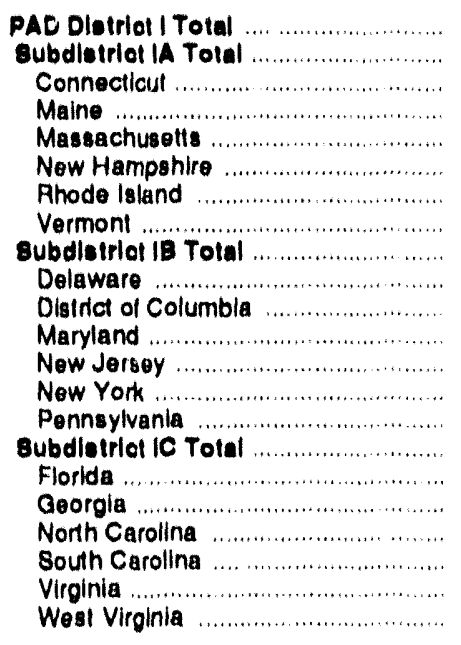 & $\begin{array}{r}284,848 \\
21,673 \\
2,224 \\
4,259 \\
3,079 \\
472 \\
10,209 \\
1,431 \\
46,758 \\
195 \\
5,557 \\
9,832 \\
4,058 \\
24,514 \\
2,602 \\
216,414 \\
8,767 \\
11,186 \\
9,042 \\
113,964 \\
73,367 \\
86\end{array}$ & $\begin{array}{r}147,482 \\
18,326 \\
6,073 \\
3,301 \\
3,806 \\
2,032 \\
3,061 \\
53 \\
71,832 \\
2,025 \\
7,064 \\
25,472 \\
25,086 \\
7,983 \\
4,202 \\
87,328 \\
9,599 \\
9,741 \\
8,014 \\
3,492 \\
25,276 \\
1,203\end{array}$ & $\begin{array}{r}468,396 \\
60,026 \\
17,042 \\
8,167 \\
20,102 \\
8,283 \\
2,403 \\
4,029 \\
185,638 \\
4,719 \\
80 \\
23,428 \\
37,754 \\
44,284 \\
75,374 \\
220,731 \\
55,445 \\
51,947 \\
32,602 \\
24,202 \\
34,649 \\
21,886\end{array}$ & $\begin{array}{r}480,043 \\
57,690 \\
11,590 \\
10,179 \\
18,229 \\
7,999 \\
2,805 \\
6,888 \\
213,728 \\
3,566 \\
549 \\
22,760 \\
28,631 \\
50,644 \\
107,575 \\
208,628 \\
65,585 \\
53,738 \\
30,305 \\
20,554 \\
28,769 \\
9,677\end{array}$ & $\begin{array}{l}- \\
- \\
- \\
- \\
- \\
- \\
- \\
- \\
- \\
- \\
- \\
- \\
- \\
- \\
- \\
- \\
- \\
-\end{array}$ & $\begin{array}{r}344 \\
53 \\
+ \\
- \\
- \\
- \\
53 \\
- \\
- \\
- \\
- \\
- \\
- \\
290 \\
24 \\
266 \\
- \\
- \\
-\end{array}$ & $\begin{array}{r}17,640,169 \\
3,764,473 \\
944,354 \\
490,919 \\
1,572,612 \\
312,652 \\
242,152 \\
201,785 \\
7,896,181 \\
157,657 \\
106,425 \\
775,120 \\
1,548,301 \\
2,865,258 \\
2,443,420 \\
3,979,818 \\
1,377,077 \\
1,176,068 \\
1,070,875 \\
689,834 \\
1,223,897 \\
441,764\end{array}$ & $\begin{array}{r}18,760,946 \\
4,147,808 \\
1,083,563 \\
526,409 \\
1,714,133 \\
322,693 \\
261,153 \\
239,853 \\
8,418,773 \\
151,061 \\
91,592 \\
845,887 \\
1,613,569 \\
3,143,476 \\
2,573,100 \\
6,194,385 \\
1,573,891 \\
1,197,651 \\
1,163,044 \\
608,266 \\
1,214,165 \\
437,348\end{array}$ \\
\hline 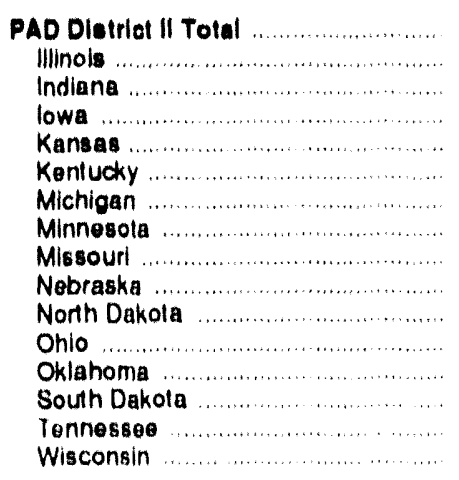 & $\begin{array}{r}44,160 \\
1,002 \\
5,596 \\
346 \\
2,074 \\
6,865 \\
1,252 \\
1,481 \\
5,294 \\
2,470 \\
1,118 \\
10,614 \\
2,411 \\
564 \\
2,177 \\
885\end{array}$ & $\begin{array}{r}88,698 \\
1,991 \\
7,444 \\
7,088 \\
19.718 \\
10,286 \\
7,061 \\
834 \\
6,376 \\
327 \\
605 \\
9.769 \\
835 \\
5,401 \\
5,983 \\
1.977\end{array}$ & $\begin{array}{r}480,377 \\
58,878 \\
28,292 \\
11,648 \\
15,040 \\
25,204 \\
59,049 \\
41,132 \\
31,187 \\
10,691 \\
5,055 \\
37,406 \\
31,149 \\
5.854 \\
35,319 \\
54,474\end{array}$ & $\begin{array}{r}487,986 \\
54,032 \\
42,988 \\
25,002 \\
17,677 \\
30,238 \\
42,212 \\
35,938 \\
37,940 \\
10,325 \\
2,218 \\
51,017 \\
19,395 \\
8,430 \\
73,011 \\
37,571\end{array}$ & $\begin{array}{r}2,381 \\
- \\
- \\
\overline{-} \\
\overline{-} \\
10 \overline{3} \\
\overline{-} \\
24 \\
\overline{8} \\
\overline{-} \\
482\end{array}$ & $\begin{array}{r}826 \\
208 \\
27 \\
11 \\
28 \\
1 \\
123 \\
\cdots \\
728\end{array}$ & $\begin{array}{r}13,069,848 \\
1,523.412 \\
1,366.108 \\
613.014 \\
661,140 \\
958.273 \\
1,060.962 \\
896.377 \\
853,995 \\
549.251 \\
312.290 \\
1.508,738 \\
596.424 \\
246.457 \\
948,462 \\
974,945\end{array}$ & $\begin{array}{r}13,746,069 \\
1,548,070 \\
1,358,418 \\
695,885 \\
644,860 \\
1,094,052 \\
1,090,880 \\
923,511 \\
947,255 \\
599,980 \\
299,822 \\
1,626,559 \\
691,927 \\
237,397 \\
1,020,640 \\
966,805\end{array}$ \\
\hline 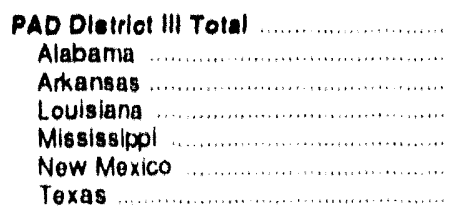 & $\begin{array}{r}188,044 \\
3,980 \\
328 \\
13,524 \\
4,404 \\
990 \\
161,817\end{array}$ & $\begin{array}{r}188,467 \\
5,063 \\
449 \\
6,640 \\
14,974 \\
2,674 \\
158,467\end{array}$ & $\begin{array}{r}299,090 \\
41.397 \\
18.901 \\
49.057 \\
19.198 \\
24.929 \\
145.607\end{array}$ & $\begin{array}{r}366,326 \\
55.323 \\
43,497 \\
43,400 \\
27.391 \\
17,237 \\
179.479\end{array}$ & $\begin{array}{l}6 \\
6 \\
\cdots\end{array}$ & $\begin{array}{r}4,343 \\
649 \\
3894\end{array}$ & $\begin{array}{r}6,638,071 \\
890,034 \\
527,091 \\
1,201,394 \\
570,635 \\
357,332 \\
3,083,485\end{array}$ & $\begin{array}{r}6,889,807 \\
930,873 \\
594,811 \\
1,110,542 \\
571,095 \\
374,795 \\
3,307,690\end{array}$ \\
\hline 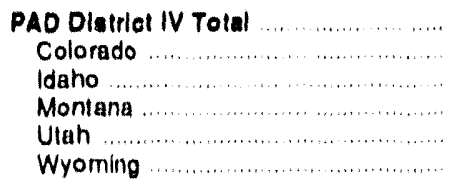 & $\begin{array}{r}9,998 \\
6,751 \\
902 \\
289 \\
1,922 \\
136\end{array}$ & $\begin{array}{r}15,560 \\
6,950 \\
4,129 \\
1,113 \\
1,693 \\
1,675\end{array}$ & $\begin{array}{r}104,736 \\
34,638 \\
20.493 \\
13.812 \\
16.220 \\
19.573\end{array}$ & $\begin{array}{l}97,499 \\
31,569 \\
13,288 \\
18.354 \\
17,691 \\
16,597\end{array}$ & $\begin{array}{c}12 \\
0 \\
9 \\
4\end{array}$ & $\begin{array}{l}79 \\
24 \\
55 \\
\cdots\end{array}$ & $\begin{array}{r}1,692,198 \\
444,252 \\
314,470 \\
305,464 \\
297,435 \\
330,577\end{array}$ & $\begin{array}{r}1,720,814 \\
476,861 \\
276,537 \\
294,967 \\
314,879 \\
357,570\end{array}$ \\
\hline 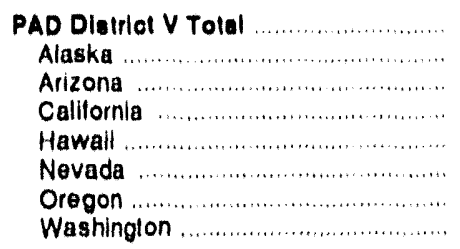 & $\begin{array}{r}287,668 \\
17,379 \\
1,416 \\
189,203 \\
65,520 \\
2,352 \\
707 \\
11,091\end{array}$ & $\begin{array}{r}215,302 \\
23,039 \\
1,729 \\
164,303 \\
9,438 \\
3,106 \\
2,125 \\
11,563\end{array}$ & $\begin{array}{r}320,962 \\
13,009 \\
38,320 \\
151,514 \\
13,859 \\
16,332 \\
34,289 \\
53,538\end{array}$ & $\begin{array}{r}325,924 \\
20,339 \\
26,148 \\
147,155 \\
10,799 \\
21,288 \\
38,275 \\
61,919\end{array}$ & 14 & $\begin{array}{c}37 \\
\cdots \\
11 \\
15 \\
15\end{array}$ & $\begin{array}{r}6,169,582 \\
417,167 \\
432,833 \\
3,181,248 \\
308,477 \\
301,666 \\
682,758 \\
845,403\end{array}$ & $\begin{array}{r}8,143,918 \\
512,265 \\
493,718 \\
3,000,408 \\
255,352 \\
334,001 \\
700,987 \\
847,187\end{array}$ \\
\hline
\end{tabular}

- Estimatod dala. Annual estimate is based on six months of dala Dashes $(\cdot)$ - No data reported.

Nole: Totals may not equal sum of components due to independent rounding

Sources: - Energy Intormation Administration Form ElA.821, "Annual Fuel Oil and Kerosene Sales Report." lor 1991 and 1992

- On-Hlghway Diesel data are Federal Highway Administation statistics of highway special luels use. 
Table 5. Sales of Residual Fuel Oll by End Use, 1991 and 1992

(Thousand Gallons)

\begin{tabular}{|c|c|c|c|c|c|c|c|c|}
\hline \multirow{2}{*}{ Dostinatlon } & \multicolumn{2}{|c|}{ Commorolal } & \multicolumn{2}{|c|}{ Induetrlel } & \multicolumn{2}{|c|}{ Oll Company } & \multicolumn{2}{|c|}{ Elootrlo Uillity } \\
\hline & 1001 & 1902 & 1901 & 1992 & 1991 & 1092 & 1001 & 1992 \\
\hline 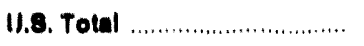 & $1,423,248$ & $1,170,049$ & $1,934,703$ & $2,126,867$ & 300,939 & 271,070 & $7,071,264$ & $0,081,441$ \\
\hline 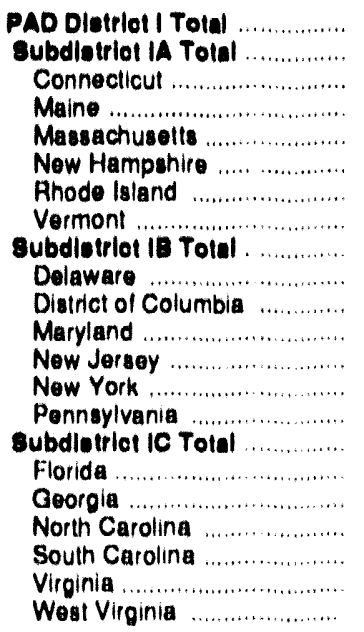 & $\begin{array}{r}1,300,180 \\
378,884 \\
22,225 \\
103,470 \\
191,583 \\
28,362 \\
24,714 \\
5,520 \\
928,311 \\
2,150 \\
9,323 \\
5,606 \\
67,481 \\
718,192 \\
26,559 \\
103,988 \\
90,139 \\
908 \\
4,942 \\
1,036 \\
4,821 \\
2,140\end{array}$ & $\begin{array}{r}1,087,183 \\
283,688 \\
34,637 \\
48,602 \\
143,494 \\
12,604 \\
20,242 \\
4,106 \\
738,398 \\
3,454 \\
10,404 \\
18,486 \\
53,029 \\
616,789 \\
34,232 \\
87,203 \\
69,753 \\
244 \\
4,344 \\
2,038 \\
8,673 \\
2,151\end{array}$ & $\begin{array}{r}1,368,896 \\
368,277 \\
41,688 \\
223,857 \\
59,057 \\
19,371 \\
15,897 \\
5,507 \\
385,041 \\
26,628 \\
54 \\
30,329 \\
128,592 \\
98,142 \\
101,296 \\
816,278 \\
109,621 \\
73,342 \\
220,235 \\
67,284 \\
102,994 \\
42,801\end{array}$ & $\begin{array}{r}1,688,182 \\
428,608 \\
47,293 \\
232,821 \\
84,287 \\
39,883 \\
17,775 \\
6,550 \\
381,492 \\
31,966 \\
76 \\
39,622 \\
118,070 \\
115,993 \\
75,766 \\
788,082 \\
169,471 \\
132,116 \\
261,275 \\
69,153 \\
112,687 \\
20,321\end{array}$ & $\begin{array}{r}108,790 \\
228 \\
225 \\
- \\
- \\
- \\
- \\
107,022 \\
13,086 \\
2,300 \\
3,411 \\
1,938 \\
86,287 \\
1,844 \\
96 \\
4 ? \\
\ldots \\
160 \\
1,246 \\
\ldots\end{array}$ & $\begin{array}{r}112,028 \\
207 \\
207 \\
\cdots \\
- \\
- \\
- \\
110,886 \\
15,813 \\
1,871 \\
2,331 \\
3,674 \\
86,765 \\
1,763 \\
115 \\
262 \\
- \\
177 \\
1,209 \\
\cdots\end{array}$ & $\begin{array}{r}6,321,428 \\
1,878,864 \\
468,860 \\
87,936 \\
931,246 \\
81,270 \\
9,212 \\
121 \\
2,610,692 \\
103,812 \\
267 \\
267,100 \\
361,590 \\
1,911,519 \\
166,670 \\
1,932,081 \\
1,840,430 \\
\ldots \\
750 \\
\ldots \\
90,901 \\
\ldots\end{array}$ & $\begin{array}{r}8,366,678 \\
1,486,283 \\
3 / 6,785 \\
100,310 \\
863,872 \\
107,873 \\
6,248 \\
84 \\
1,862,074 \\
96,185 \\
172,069 \\
106,238 \\
1,342,151 \\
145,432 \\
2,049,318 \\
1,073,541 \\
\ldots \\
1,015 \\
74,762 \\
-\end{array}$ \\
\hline 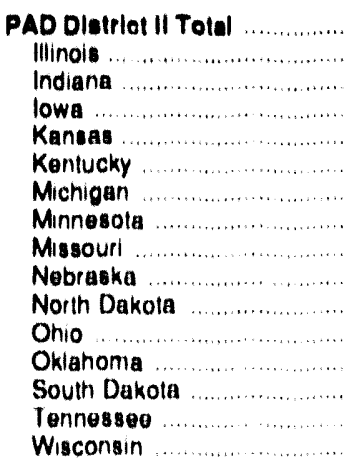 & $\begin{array}{r}40,738 \\
1,648 \\
8,509 \\
376 \\
2.2 \\
201 \\
12,378 \\
1,246 \\
1,117 \\
317 \\
1,692 \\
3,203 \\
1,470 \\
695 \\
1,505\end{array}$ & $\begin{array}{r}32,001 \\
1,679 \\
695 \\
1,440 \\
847 \\
- \\
458 \\
7,630 \\
120 \\
1,886 \\
468 \\
2,859 \\
1,674 \\
1,378 \\
2,221 \\
8,945\end{array}$ & $\begin{array}{r}208,678 \\
10,455 \\
70,048 \\
3,670 \\
2,674 \\
10,118 \\
26,501 \\
20,709 \\
19,996 \\
1,152 \\
4,048 \\
44,570 \\
6,461 \\
1,354 \\
14,129 \\
23,193\end{array}$ & $\begin{array}{r}316,437 \\
7,705 \\
96,038 \\
2,692 \\
4,657 \\
8,172 \\
28,432 \\
26,784 \\
23,669 \\
5,641 \\
3,943 \\
51,868 \\
22,213 \\
4,207 \\
11,165 \\
19,251\end{array}$ & $\begin{array}{r}121,717 \\
25,298 \\
53,590 \\
-2 \overline{3} \\
2,283 \\
9,114 \\
4,914 \\
10,693 \\
8,486 \\
2,797 \\
\cdots \\
111 \\
4,423\end{array}$ & $\begin{array}{r}98,127 \\
6,708 \\
54,236 \\
1,413 \\
8,132 \\
1,024 \\
11,442 \\
358 \\
6,830 \\
3,423 \\
2 \\
241 \\
4,321\end{array}$ & $\begin{array}{r}137,244 \\
81,324 \\
\\
35,348 \\
470 \\
11,912 \\
305 \\
7,883 \\
1 \\
1\end{array}$ & $\begin{array}{r}138,094 \\
04,468 \\
21,265 \\
15,734 \\
420 \\
207 \\
\end{array}$ \\
\hline 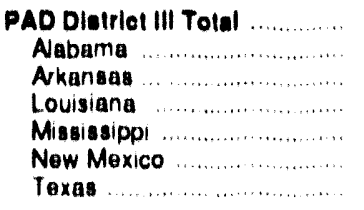 & $\begin{array}{r}24,487 \\
10.231 \\
5.078 \\
25 \\
9.123\end{array}$ & $\begin{array}{r}1,018 \\
186 \\
234 \\
17 \\
611\end{array}$ & $\begin{array}{r}117,327 \\
3,514 \\
6,000 \\
47,234 \\
10,001 \\
4,916 \\
45,512\end{array}$ & $\begin{array}{r}96,194 \\
14,349 \\
1,039 \\
38,778 \\
7,414 \\
4,944 \\
29,670\end{array}$ & $\begin{array}{r}707 \\
\cdots \\
\cdots \\
\cdots \\
\cdots \\
\cdots\end{array}$ & $\begin{array}{r}2,119 \\
2.119 \\
2\end{array}$ & $\begin{array}{r}26,919 \\
49 \\
1,584 \\
25,286 \\
\end{array}$ & $\begin{array}{r}26,133 \\
743 \\
24,390 \\
\end{array}$ \\
\hline 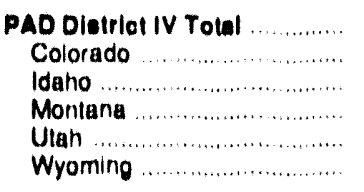 & $\begin{array}{r}1,198 \\
0 \\
52 \\
138 \\
963 \\
45\end{array}$ & $\begin{array}{r}1,498 \\
9 \\
536 \\
151 \\
799 \\
-\end{array}$ & $\begin{array}{r}0,604 \\
1,416 \\
1,809 \\
228 \\
3,093 \\
58\end{array}$ & $\begin{array}{r}6,868 \\
145 \\
307 \\
3,307 \\
2,531 \\
266\end{array}$ & $\begin{array}{r}11,700 \\
5,762 \\
4,404 \\
1,595\end{array}$ & $\begin{array}{r}6,342 \\
\ldots \\
6,259 \\
82\end{array}$ & $\begin{array}{l}- \\
- \\
\cdots \\
\cdots \\
-\end{array}$ & $\begin{array}{l}- \\
\cdots \\
\cdots \\
\cdots \\
\cdots\end{array}$ \\
\hline 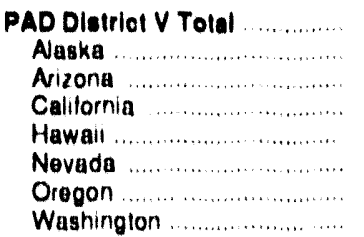 & $\begin{array}{r}48,376 \\
462 \\
32,074 \\
780 \\
97 \\
10,734 \\
4,229\end{array}$ & $\begin{array}{r}64,361 \\
1,652 \\
41,123 \\
15 \\
9,385 \\
2,176\end{array}$ & $\begin{array}{r}178,688 \\
11,752 \\
1,407 \\
53,936 \\
35,696 \\
3,431 \\
14,565 \\
51,802\end{array}$ & $\begin{array}{r}142,488 \\
11,669 \\
3,638 \\
53,763 \\
16,254 \\
3,093 \\
10,466 \\
34,616\end{array}$ & $\begin{array}{r}67,064 \\
20,041 \\
33,455 \\
4,468\end{array}$ & $\begin{array}{r}61,988 \\
10.208 \\
28,838 \\
3,91 ?\end{array}$ & $\begin{array}{r}588,684 \\
100,617 \\
474,836 \\
10,151 \\
\ldots\end{array}$ & $\begin{array}{r}431,840 \\
76 \\
413,179 \\
18,285 \\
\end{array}$ \\
\hline
\end{tabular}

Seo lootnotes at end of table. 
Table 5. Sales of Resldual Fuel Oll by End Use, 1991 and 1992 (Continued) (Thousand Gallons)

\begin{tabular}{|c|c|c|c|c|c|c|c|c|}
\hline \multirow{2}{*}{ Destinatlon } & \multicolumn{2}{|c|}{ Voesol Bunkering } & \multicolumn{2}{|c|}{ Milltary } & \multicolumn{2}{|c|}{ All Other } & \multicolumn{2}{|c|}{ Total } \\
\hline & 1991 & 1092 & 1991 & 1992 & 1991 & 1092 & 1991 & 1892 \\
\hline 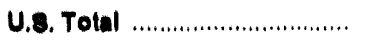 & $6,773,174$ & $0,563,220$ & 119,976 & 91,701 & 7,738 & 8,613 & $1-831,823$ & $16,199,042$ \\
\hline 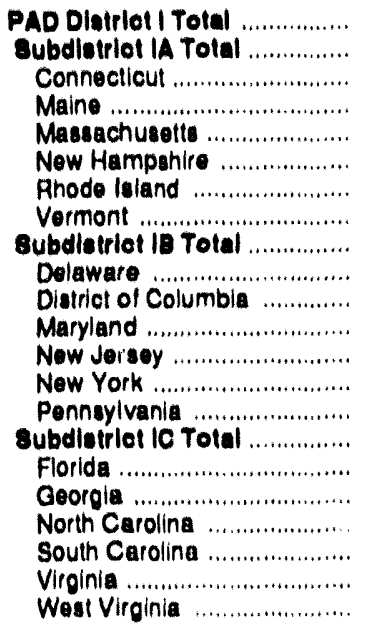 & 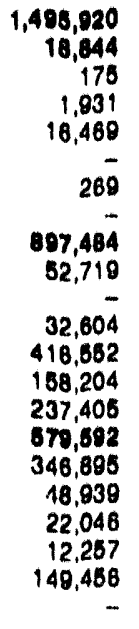 & $\begin{array}{r}1,634,340 \\
14,708 \\
881 \\
71 \\
71,874 \\
13,84 \\
140 \\
- \\
58- \\
886,216 \\
38,427 \\
48,684 \\
368,785 \\
136,307 \\
268,043 \\
661,380 \\
398,141 \\
130,828 \\
14,738 \\
15,734 \\
101,221 \\
-\end{array}$ & $\begin{array}{r}111,164 \\
17,376 \\
3,688 \\
2,939 \\
2,137 \\
8,415 \\
110 \\
107 \\
80,802 \\
2,641 \\
26,04 \overline{9} \\
11,931 \\
8,677 \\
2,604 \\
43,188 \\
3,633 \\
9- \\
9,295 \\
20,982 \\
9,275 \\
-\end{array}$ & $\begin{array}{r}79,334 \\
16,969 \\
1,027 \\
5,968 \\
2,924 \\
4,681 \\
2,291 \\
172 \\
34,861 \\
1,691 \\
278 \\
14,403 \\
7,877 \\
7,020 \\
2,396 \\
27,814 \\
2,318 \\
-10, \\
10,756 \\
4,904 \\
9,836 \\
-\end{array}$ & $\begin{array}{r}689 \\
\overline{-} \\
\overline{-} \\
\overline{-} \\
27 \overline{1} \\
167 \\
\overline{-} \\
104 \\
\overline{-} \\
38 \overline{-} \\
24 \\
\overline{-} \\
363 \\
\overline{-}\end{array}$ & $\begin{array}{r}181 \\
18 \\
18 \\
- \\
= \\
\overline{-} \\
\overline{-} \\
117 \\
= \\
\overline{-} \\
32 \\
80 \\
46 \\
- \\
45 \\
- \\
-\end{array}$ & 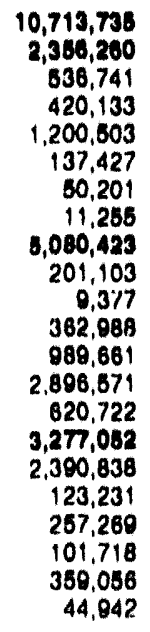 & 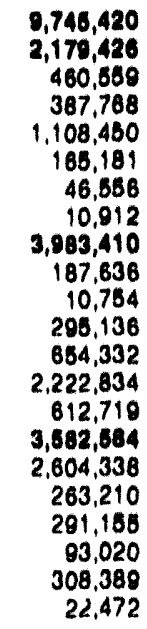 \\
\hline 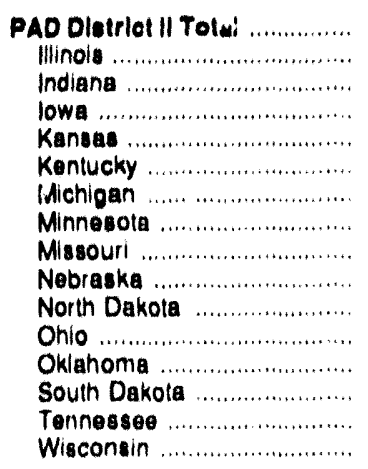 & $\begin{array}{r}8,020 \\
863 \\
3,762 \\
- \\
- \\
- \\
2,090 \\
46 \\
- \\
- \\
328 \\
\overline{-} \\
2,11 \overline{-} \\
12\end{array}$ & $\begin{array}{r}17,068 \\
1,221 \\
8,041 \\
= \\
= \\
3,785 \\
100 \\
671 \\
= \\
2,113 \\
= \\
1,713 \\
314\end{array}$ & $\begin{array}{l}82 \\
= \\
= \\
= \\
11 \\
11 \\
= \\
= \\
= \\
= \\
=\end{array}$ & $\begin{array}{l}= \\
= \\
= \\
= \\
= \\
= \\
= \\
= \\
- \\
-\end{array}$ & $\begin{array}{r}343 \\
= \\
= \\
= \\
107 \\
238 \\
= \\
= \\
= \\
= \\
=\end{array}$ & $\begin{array}{r}380 \\
- \\
- \\
- \\
50 \\
- \\
- \\
- \\
131 \\
- \\
\overline{-} \\
178\end{array}$ & $\begin{array}{r}874,721 \\
110,288 \\
136,009 \\
4,047 \\
6,249 \\
19,231 \\
69,172 \\
44,602 \\
33,164 \\
8,269 \\
13,155 \\
57,270 \\
9,664 \\
2,825 \\
17,053 \\
35,733\end{array}$ & $\begin{array}{r}802,986 \\
111,781 \\
169,010 \\
4,132 \\
6,917 \\
16,304 \\
61,026 \\
46,068 \\
40,652 \\
7,226 \\
11,681 \\
60,800 \\
23,887 \\
5,586 \\
15,341 \\
33,008\end{array}$ \\
\hline 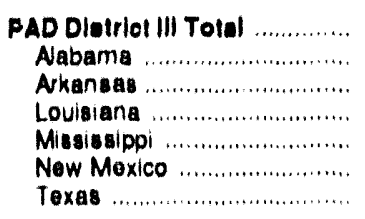 & $\begin{array}{r}2,483,074 \\
134,259 \\
1,042,062 \\
168,370 \\
1,141,39 \overline{4}\end{array}$ & $\begin{array}{r}2,626,137 \\
136,732 \\
1,130,126 \\
101,226 \\
1,167,054\end{array}$ & $\begin{array}{r}1,184 \\
1,184 \\
- \\
- \\
- \\
-\end{array}$ & $\begin{array}{l}- \\
z \\
z \\
-\end{array}$ & $\begin{array}{l}61 \\
- \\
= \\
01\end{array}$ & $\begin{array}{l}- \\
= \\
= \\
=\end{array}$ & $\begin{array}{r}2,864,620 \\
149,248 \\
6,138 \\
1,096,849 \\
200,682 \\
4,977 \\
1,196,736\end{array}$ & $\begin{array}{r}2,649,001 \\
151,081 \\
1,198 \\
1,169,881 \\
133,047 \\
4,944 \\
1,189,464\end{array}$ \\
\hline 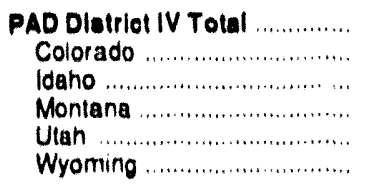 & $\begin{array}{l}- \\
- \\
- \\
-\end{array}$ & $\begin{array}{l}- \\
- \\
-\end{array}$ & $\begin{array}{l}- \\
- \\
- \\
- \\
-\end{array}$ & $\begin{array}{l}- \\
- \\
- \\
-\end{array}$ & $\begin{array}{l}- \\
- \\
-\end{array}$ & $\begin{array}{l}38 \\
- \\
= \\
\overline{38}\end{array}$ & $\begin{array}{r}19,682 \\
1,416 \\
1,861 \\
6,127 \\
8,460 \\
1,698\end{array}$ & $\begin{array}{r}14,429 \\
154 \\
843 \\
3,458 \\
9,589 \\
386\end{array}$ \\
\hline 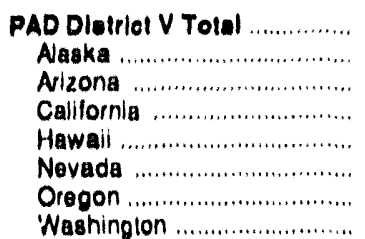 & $\begin{array}{r}2,784,360 \\
3,073 \\
- \\
1,768,856 \\
105,866 \\
- \\
240,505 \\
666,360\end{array}$ & $\begin{array}{r}2,488,778 \\
12,236 \\
1,247,458 \\
138,828 \\
=- \\
225,162 \\
862,102\end{array}$ & $\begin{array}{r}7,847 \\
- \\
3,712 \\
\overline{73} \\
3,762\end{array}$ & $\begin{array}{r}12,467 \\
- \\
- \\
862 \\
8,058 \\
\overline{55} \\
3,491\end{array}$ & $\begin{array}{r}6,676 \\
- \\
5,600 \\
\overline{7} \\
7\end{array}$ & $\begin{array}{r}8,036 \\
- \\
83 \\
7,053 \\
- \\
-\end{array}$ & $\begin{array}{r}3,669,178 \\
14,825 \\
7,869 \\
1,975,288 \\
660,945 \\
13,679 \\
265,948 \\
730,620\end{array}$ & $\begin{array}{r}3,186,604 \\
23,005 \\
3,638 \\
1,323,083 \\
654,231 \\
21,393 \\
264,088 \\
806,296\end{array}$ \\
\hline
\end{tabular}

Dashes (.) - No dala reported.

Note: Totals may not equal sum of components due to independent rounding.

Sources: - Energy Information Administration Form EIA-821, "Annual Fuel Oil and Kerosene Sales Report," lor 1991 and 1892. 
Table 6. Sales of Kerosene by End Use, 1991 and 1992

(Thousand Gallons)

\begin{tabular}{|c|c|c|c|c|c|c|}
\hline \multirow{2}{*}{ Destination } & \multicolumn{2}{|c|}{ Rooldential } & \multicolumn{2}{|c|}{ Commerolal } & \multicolumn{2}{|c|}{ Induotrlal } \\
\hline & 1901 & 1902 & 1901 & 1092 & 1001 & 1992 \\
\hline 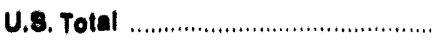 & 838,864 & 481,611 & 80,542 & 82,398 & 82,438 & 46,090 \\
\hline 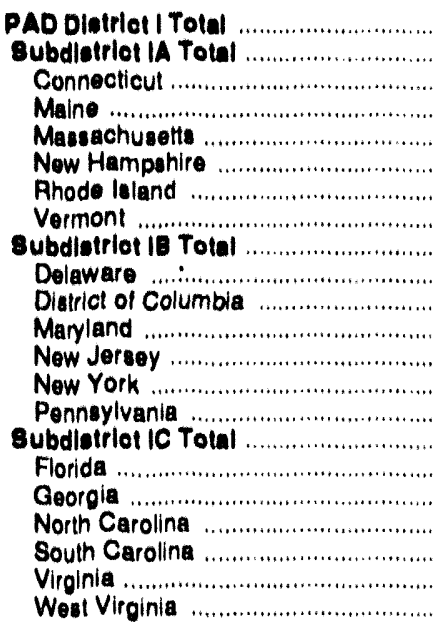 & $\begin{array}{r}428,493 \\
61,767 \\
7,333 \\
24,892 \\
6,331 \\
11,313 \\
1,488 \\
10,401 \\
189,010 \\
6,981 \\
168 \\
16,634 \\
13,810 \\
88,129 \\
83,319 \\
177,728 \\
8,197 \\
4,785 \\
70,288 \\
30,684 \\
68,625 \\
8,277\end{array}$ & $\begin{array}{r}388,784 \\
89,842 \\
8,211 \\
19,887 \\
10,897 \\
10,502 \\
1,533 \\
8,812 \\
180,089 \\
6,039 \\
148 \\
13,284 \\
11,459 \\
32,603 \\
66,555 \\
178,863 \\
11,500 \\
4,574 \\
77,044 \\
18,528 \\
53,006 \\
10,300\end{array}$ & $\begin{array}{r}64,402 \\
22,283 \\
7,020 \\
5,237 \\
8,409 \\
898 \\
48 \\
681 \\
26,381 \\
543 \\
167 \\
2,192 \\
8,050 \\
6,946 \\
5,483 \\
16,768 \\
1,214 \\
2,220 \\
3,921 \\
488 \\
6,214 \\
2,701\end{array}$ & $\begin{array}{r}61,068 \\
0,367 \\
1,894 \\
2,767 \\
3,084 \\
926 \\
130 \\
596 \\
39,773 \\
50 \\
181 \\
1,767 \\
16,348 \\
17,148 \\
4,279 \\
11,939 \\
1,246 \\
1,535 \\
1,931 \\
572 \\
5,328 \\
1,328\end{array}$ & $\begin{array}{r}31,921 \\
2,801 \\
731 \\
306 \\
437 \\
251 \\
634 \\
142 \\
17,383 \\
182 \\
- \\
648 \\
3,250 \\
8,853 \\
4,453 \\
12,037 \\
397 \\
844 \\
4,320 \\
3,472 \\
2,093 \\
910\end{array}$ & $\begin{array}{r}28,430 \\
8,404 \\
279 \\
286 \\
3,764 \\
549 \\
464 \\
92 \\
13,623 \\
80 \\
- \\
363 \\
6,057 \\
3,834 \\
3,287 \\
9,402 \\
172 \\
301 \\
3,688 \\
2,508 \\
1,176 \\
1,657\end{array}$ \\
\hline 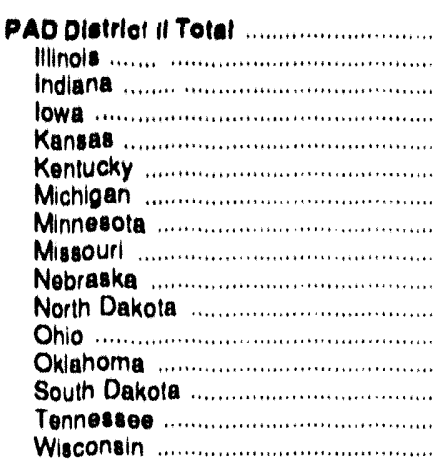 & $\begin{array}{r}02,032 \\
4,689 \\
13,265 \\
1,414 \\
418 \\
15,867 \\
11,719 \\
1,707 \\
1,558 \\
228 \\
312 \\
28,414 \\
418 \\
189 \\
11,250 \\
1,271\end{array}$ & $\begin{array}{r}86,302 \\
2,544 \\
7,793 \\
847 \\
553 \\
15,337 \\
8,595 \\
1,605 \\
868 \\
427 \\
258 \\
30,580 \\
449 \\
155 \\
15,158 \\
1,222 \\
\end{array}$ & $\begin{array}{r}18,881 \\
1,667 \\
1,811 \\
108 \\
153 \\
4,283 \\
720 \\
145 \\
185 \\
113 \\
21 \\
7,573 \\
404 \\
5 \\
1,325 \\
370\end{array}$ & $\begin{array}{r}14,288 \\
1,444 \\
2,460 \\
166 \\
188 \\
2,433 \\
213 \\
282 \\
679 \\
62 \\
14 \\
2,837 \\
154 \\
16 \\
2,884 \\
427\end{array}$ & $\begin{array}{r}11,800 \\
1,591 \\
1,673 \\
80 \\
142 \\
2,084 \\
1,579 \\
26 \\
157 \\
53 \\
2 \\
2,656 \\
258 \\
- \\
1,179 \\
139\end{array}$ & $\begin{array}{r}11,149 \\
1,389 \\
1,054 \\
51 \\
44 \\
2,622 \\
1,216 \\
14 \\
68 \\
32 \\
4 \\
4,839 \\
292 \\
11 \\
325 \\
188\end{array}$ \\
\hline $\begin{array}{l}\text { PAD Diotrlot III Total } \\
\text { Alabama } \\
\text { Arkansas } \\
\text { Loulalana } \\
\text { Mississippl } \\
\text { Now Moxico } \\
\text { Toxas }\end{array}$ & $\begin{array}{r}6,368 \\
2,583 \\
689 \\
670 \\
948 \\
257 \\
1,441\end{array}$ & $\begin{array}{r}3,724 \\
1,275 \\
308 \\
394 \\
600 \\
191 \\
956\end{array}$ & $\begin{array}{r}3.244 \\
627 \\
78 \\
919 \\
258 \\
840 \\
522\end{array}$ & $\begin{array}{r}4,978 \\
733 \\
214 \\
430 \\
368 \\
368 \\
2,866\end{array}$ & $\begin{array}{r}6,008 \\
746 \\
409 \\
1,800 \\
213 \\
1,541 \\
1,200\end{array}$ & $\begin{array}{r}4,729 \\
1,376 \\
84 \\
830 \\
436 \\
357 \\
1,646\end{array}$ \\
\hline $\begin{array}{l}\text { PAD Dlatriol IV Total } \\
\text { Colorado } \\
\text { ldaho } \\
\text { Montana } \\
\text { Ulah } \\
\text { Wyoming }\end{array}$ & $\begin{array}{r}1,448 \\
1,011 \\
66 \\
34 \\
211 \\
126\end{array}$ & $\begin{array}{r}1,778 \\
1,550 \\
68 \\
8 \\
95 \\
53\end{array}$ & $\begin{array}{r}944 \\
446 \\
8 \\
10 \\
356 \\
123\end{array}$ & $\begin{array}{r}346 \\
282 \\
7 \\
8 \\
39 \\
10\end{array}$ & $\begin{array}{r}324 \\
42 \\
57 \\
1 \\
96 \\
129\end{array}$ & $\begin{array}{r}318 \\
52 \\
11 \\
1 \\
27 \\
227\end{array}$ \\
\hline 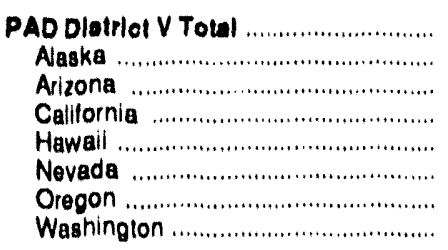 & $\begin{array}{r}6,633 \\
329 \\
29 \\
3,346 \\
431 \\
564 \\
1,933\end{array}$ & $\begin{array}{r}3,836 \\
28 \\
82 \\
1,367 \\
- \\
403 \\
716 \\
1,239\end{array}$ & $\begin{array}{r}2,070 \\
5 \\
78 \\
968 \\
6 \\
128 \\
160 \\
724\end{array}$ & $\begin{array}{r}1,748 \\
10 \\
27 \\
835 \\
3 \\
172 \\
196 \\
503\end{array}$ & $\begin{array}{r}2,584 \\
7 \\
1,351 \\
557 \\
4 \\
314 \\
101 \\
250\end{array}$ & $\begin{array}{r}1,478 \\
11 \\
14 \\
588 \\
9 \\
389 \\
309 \\
156\end{array}$ \\
\hline
\end{tabular}

See tootnotes at end of table. 
Table 6. Sales of Kerosene by End Une, 1991 and 1992 (Continued)

(Thousand Gallons)

\begin{tabular}{|c|c|c|c|c|c|c|}
\hline \multirow{2}{*}{ Destination } & \multicolumn{2}{|c|}{ Farm } & \multicolumn{2}{|c|}{ All Other } & \multicolumn{2}{|c|}{ Total } \\
\hline & 1901 & 1092 & 1901 & 1002 & 1001 & 1902 \\
\hline 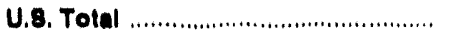 & 17,053 & 20,036 & 14,810 & 6,382 & 709,716 & 036,426 \\
\hline 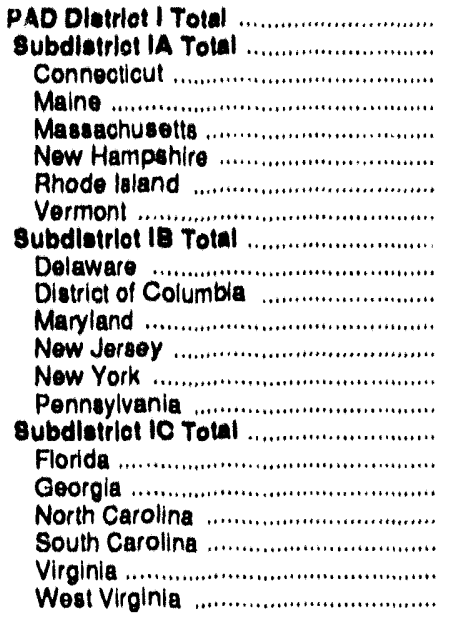 & $\begin{array}{r}8,801 \\
966 \\
2 \\
652 \\
31 \\
27 \\
1 \\
253 \\
3,703 \\
76 \\
- \\
403 \\
2 \\
2,670 \\
651 \\
4,193 \\
71 \\
98 \\
2,079 \\
773 \\
754 \\
418\end{array}$ & $\begin{array}{r}10,948 \\
488 \\
51 \\
233 \\
28 \\
48 \\
1 \\
127 \\
6,168 \\
37 \\
37 \\
347 \\
28 \\
3,782 \\
1,972 \\
4,293 \\
35 \\
43 \\
2,359 \\
150 \\
916 \\
791\end{array}$ & $\begin{array}{r}9,887 \\
2,307 \\
630 \\
115 \\
304 \\
1,041 \\
6 \\
72 \\
4,481 \\
87 \\
127 \\
732 \\
2,842 \\
903 \\
2,899 \\
76 \\
240 \\
732 \\
334 \\
1,013 \\
304\end{array}$ & $\begin{array}{r}3,687 \\
476 \\
11 \\
94 \\
63 \\
263 \\
2 \\
44 \\
2,210 \\
11 \\
101 \\
101 \\
569 \\
836 \\
692 \\
1,001 \\
177 \\
62 \\
89 \\
280 \\
255 \\
168\end{array}$ & $\begin{array}{r}843,236 \\
80,888 \\
15,916 \\
31,202 \\
15,512 \\
13,530 \\
2,176 \\
11,519 \\
239,088 \\
7,839 \\
336 \\
20,000 \\
25,844 \\
111,140 \\
74,809 \\
213,413 \\
9,854 \\
8,168 \\
81,340 \\
35,751 \\
65,508 \\
12,612\end{array}$ & $\begin{array}{r}489,013 \\
78,867 \\
10,446 \\
23,227 \\
17,807 \\
12,288 \\
2,130 \\
9,870 \\
211,869 \\
6,217 \\
329 \\
15,864 \\
34,481 \\
78,204 \\
76,785 \\
202,488 \\
13,131 \\
6,614 \\
85,111 \\
22,009 \\
61,679 \\
14,145\end{array}$ \\
\hline 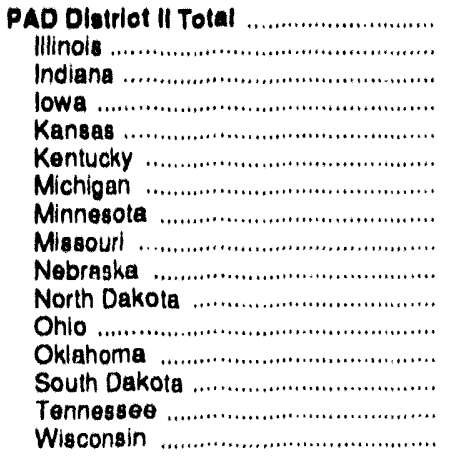 & $\begin{array}{r}5,417 \\
253 \\
229 \\
454 \\
287 \\
486 \\
458 \\
385 \\
114 \\
323 \\
69 \\
1,831 \\
97 \\
109 \\
181 \\
141\end{array}$ & $\begin{array}{r}6,737 \\
298 \\
1,060 \\
669 \\
445 \\
575 \\
461 \\
297 \\
163 \\
309 \\
14 \\
1,715 \\
308 \\
144 \\
81 \\
200\end{array}$ & $\begin{array}{r}3,161 \\
117 \\
88 \\
100 \\
22 \\
442 \\
637 \\
16 \\
701 \\
13 \\
11 \\
313 \\
137 \\
5 \\
429 \\
130\end{array}$ & $\begin{array}{r}1,437 \\
273 \\
136 \\
27 \\
148 \\
252 \\
41 \\
27 \\
37 \\
2 \\
1 \\
173 \\
103 \\
- \\
114 \\
105\end{array}$ & $\begin{array}{r}131,091 \\
8,527 \\
17,065 \\
2,136 \\
1,022 \\
23,162 \\
15,114 \\
2,278 \\
2,715 \\
732 \\
415 \\
40,788 \\
1,314 \\
307 \\
14,364 \\
2,051\end{array}$ & $\begin{array}{r}119,972 \\
5,947 \\
12,502 \\
1,769 \\
1,378 \\
21,218 \\
10,525 \\
2,225 \\
1,814 \\
833 \\
291 \\
39,144 \\
1,305 \\
327 \\
18,563 \\
2,142\end{array}$ \\
\hline 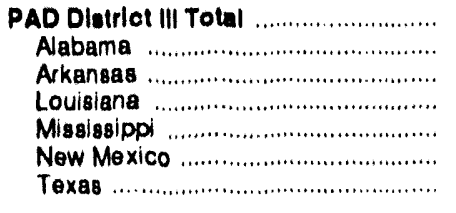 & $\begin{array}{r}1,444 \\
8 \\
210 \\
102 \\
787 \\
48 \\
288\end{array}$ & $\begin{array}{r}1,704 \\
41 \\
190 \\
205 \\
37 \\
15 \\
1,216\end{array}$ & $\begin{array}{r}1,396 \\
119 \\
222 \\
180 \\
370 \\
36 \\
469\end{array}$ & $\begin{array}{r}1,056 \\
68 \\
112 \\
85 \\
168 \\
30 \\
593\end{array}$ & $\begin{array}{r}16,460 \\
4,053 \\
1,508 \\
3,672 \\
2,576 \\
2,722 \\
3,821\end{array}$ & $\begin{array}{r}16,190 \\
3,493 \\
907 \\
1,943 \\
1,609 \\
961 \\
7,278\end{array}$ \\
\hline 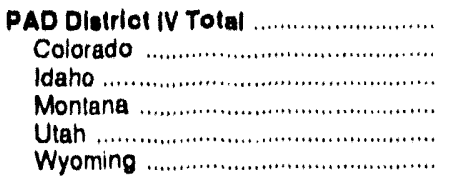 & $\begin{array}{r}384 \\
463 \\
19 \\
76 \\
11 \\
16\end{array}$ & $\begin{array}{r}265 \\
234 \\
11 \\
1 \\
13 \\
7\end{array}$ & $\begin{array}{r}232 \\
169 \\
- \\
4 \\
36 \\
3\end{array}$ & $\begin{array}{r}42 \\
29 \\
4 \\
6 \\
1 \\
2\end{array}$ & $\begin{array}{r}3,533 \\
2,150 \\
150 \\
126 \\
710 \\
397\end{array}$ & $\begin{array}{r}2,746 \\
2,147 \\
102 \\
24 \\
175 \\
299\end{array}$ \\
\hline 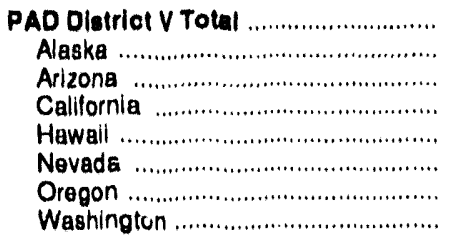 & $\begin{array}{r}746 \\
\overline{8} \\
667 \\
\overline{20} \\
35 \\
16\end{array}$ & $\begin{array}{r}384 \\
\overline{6} \\
233 \\
- \\
7 \\
51 \\
87\end{array}$ & $\begin{array}{r}473 \\
- \\
58 \\
306 \\
- \\
56 \\
31 \\
22\end{array}$ & $\begin{array}{r}161 \\
3 \\
1 \\
128 \\
- \\
5 \\
23 \\
2\end{array}$ & $\begin{array}{r}12,607 \\
341 \\
1,524 \\
5,846 \\
11 \\
949 \\
891 \\
2,945\end{array}$ & $\begin{array}{r}7,604 \\
52 \\
130 \\
3,152 \\
13 \\
976 \\
1,296 \\
1,887\end{array}$ \\
\hline
\end{tabular}

Dashes $(\cdot)$ - No data reported.

Note: Totals may not equal sum of components due to independent rounding.

Sources: - Energy Intormation Administration Form ElA-821, "Annual Fuel Oil and Kerosene Sales Report," lor 1991 and 1992. 
Table 7. Sales for Residential Use: Distillate Fuel Oll and Kerosene, 1992 (Thousand Gallons)

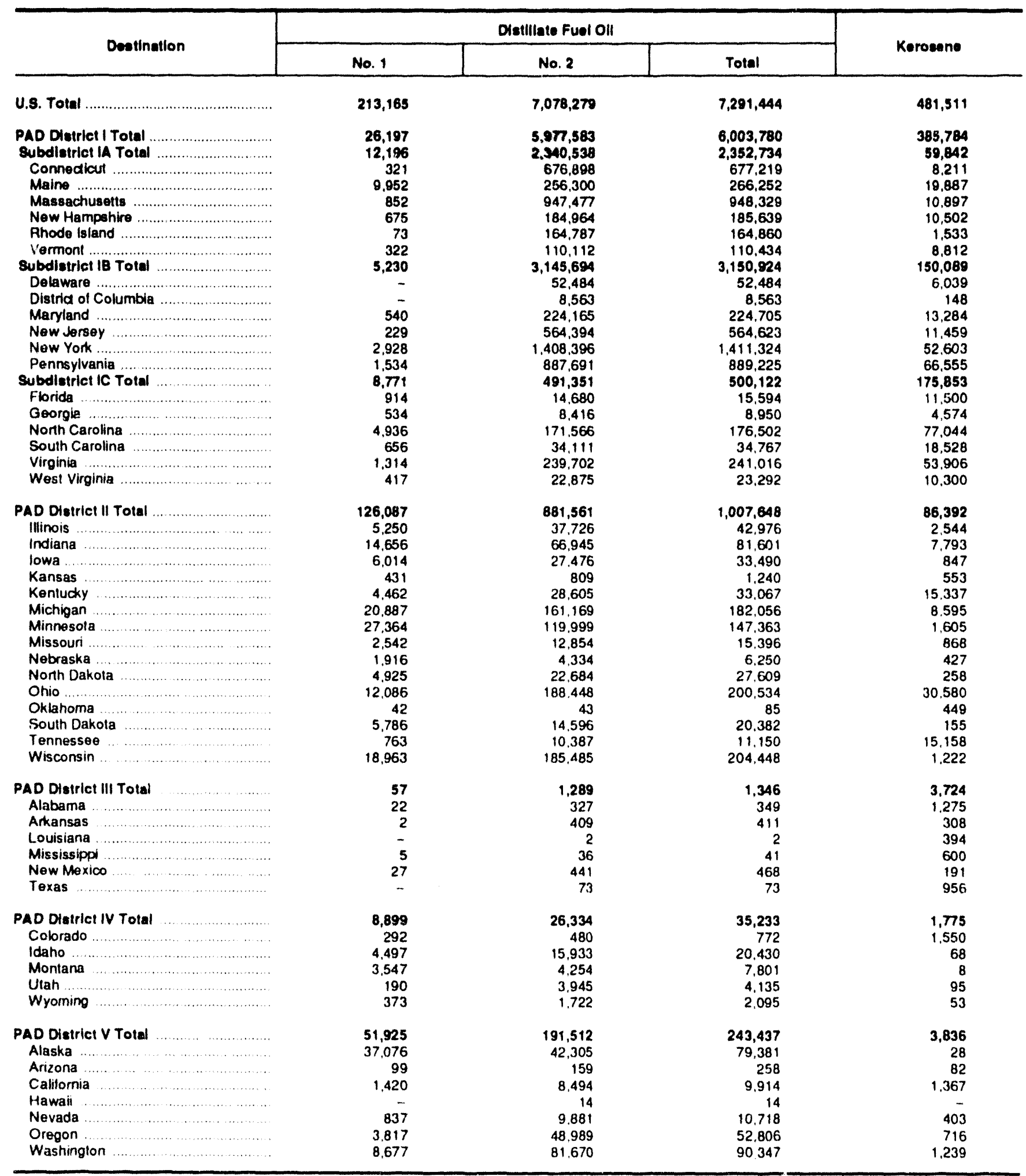

Dashes $(-)$ = No data reported.

Nole Totals may not equal sum of components due to independent rounding

Sources: "Energy Intormation Administralion Form EIA-821. "Annual Fuel Oil and Kerosene Sales Report," lor 1992

- Kerosene data are based on data from the Energy Inlormation Administration's "Petroleum Supply Ainnual." Volume 1, 1992. (DOEJEIA-0340(92)/1) 
Table 8. Sales for Commerclal Use: Distlllate Fuel Oll, Residual Fuel Oll, and Kerosene, 1992 (Thousand Gallons)

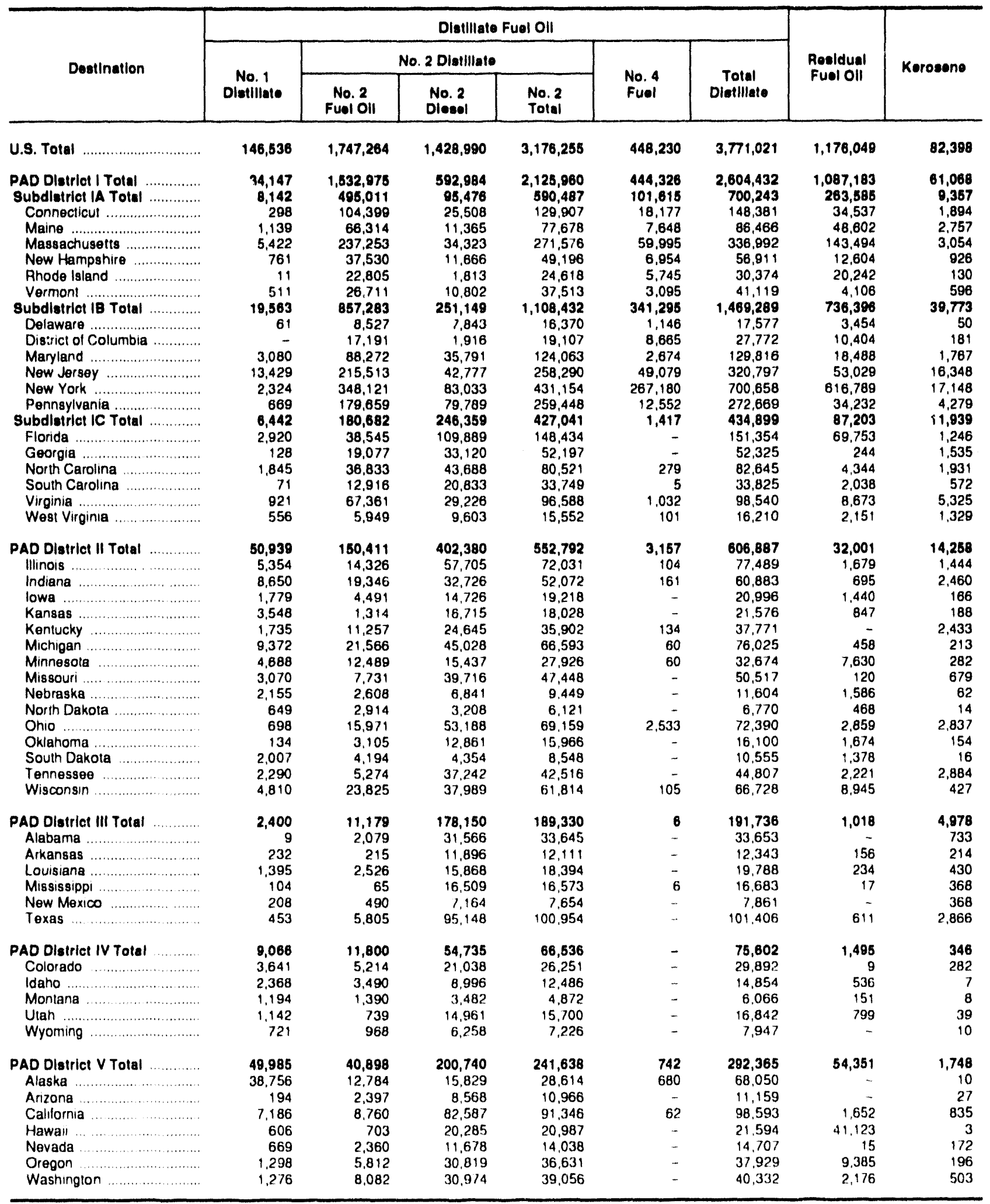

Dashes $(\cdot)$ = No dala reported

Note: Totals miay not equal sum ol components due to independent rounding

Sources: - Energy Intormation Administration Form EIA 821. "Annual Fuel Oit and Kerosene Sales Report," for 1992

- Kerosene data are based on data from the Energy Inlormation Adminisiration's "Petroleum Supply Annual," Volume 1, 1992, (DOE/EIA 0340(92)/1) 
Table 9. 3ales for Industrial Use and for Oil Company Use: Distillate Fuel OII, Residual Fuel Oil, and Kerosene, 1992 (Thousand Gallons)

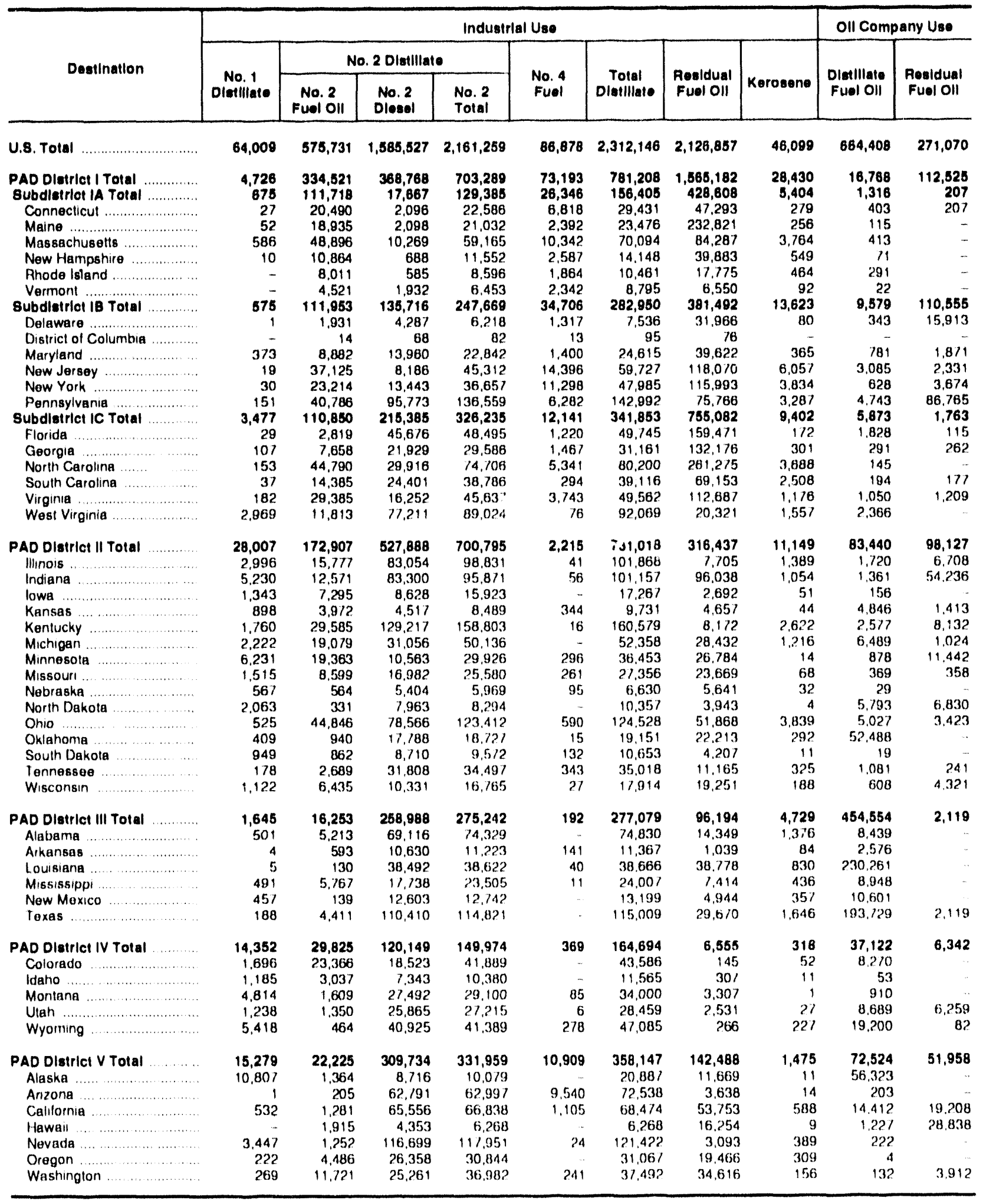

Dashes $(-)$. No data reported

Note Totals may not equal sum of components due to independent rounding.

Sources: - Energy Intormation Administration Form EIA 821. "Anmual Fuel Oil and Kerosene Sales Report," for 199?

- Kerosene data are based on data from the Energy Information Admunistration's "Petroleum Supply Annual," Volume 1, 1992. (DOE/E $\mid A-0340(92) / 1)$ 
Table 10. Sales for Farm Use: Distillate Fuel Oll and Kerosene; Sales for Electric Utility Use: Distillate Fuel Oll and Residual Fuel OII, 1992 (Thousand Gallons)

\begin{tabular}{|c|c|c|c|c|c|c|}
\hline \multirow[b]{2}{*}{ Destination } & \multicolumn{4}{|c|}{ Farm } & \multicolumn{2}{|c|}{ Electrilo } \\
\hline & Dlesel & $\begin{array}{l}\text { Other } \\
\text { Distlliate }\end{array}$ & $\begin{array}{c}\text { Total } \\
\text { Distillate }\end{array}$ & Kerosene & $\begin{array}{l}\text { Diatlliate } \\
\text { Fuel Oll }\end{array}$ & $\begin{array}{l}\text { Posldual } \\
\text { Fuol Oll }\end{array}$ \\
\hline 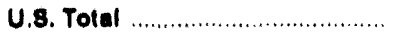 & $3,282,832$ & 246,086 & $3,499,618$ & 20,036 & 840,683 & $8,061,441$ \\
\hline 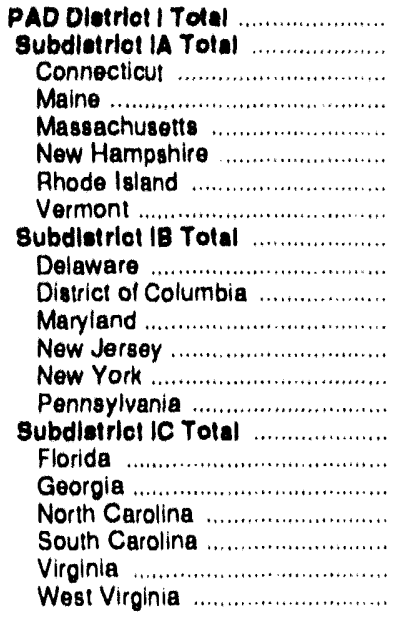 & $\begin{array}{r}362,011 \\
18,814 \\
1,887 \\
2,504 \\
2,204 \\
1,370 \\
310 \\
7,540 \\
107,884 \\
5,609 \\
20,506 \\
6,335 \\
37,207 \\
37,906 \\
238,633 \\
65,222 \\
55,556 \\
46,404 \\
28,438 \\
38,137 \\
4,875\end{array}$ & $\begin{array}{r}78,167 \\
12,170 \\
3,688 \\
1,639 \\
2,556 \\
1,633 \\
330 \\
2,323 \\
48,762 \\
358 \\
- \\
2,309 \\
3,107 \\
20,643 \\
22,345 \\
17,235 \\
7 \\
1 \\
4,494 \\
1,377 \\
9,861 \\
1,494\end{array}$ & $\begin{array}{r}440,178 \\
27,084 \\
5,575 \\
4,143 \\
4,760 \\
3,003 \\
641 \\
9,863 \\
156,326 \\
5,967 \\
- \\
22,815 \\
9,442 \\
57,850 \\
60,250 \\
255,868 \\
65,230 \\
55,557 \\
50,898 \\
29,816 \\
47,997 \\
6,370\end{array}$ & $\begin{array}{r}10,945 \\
488 \\
51 \\
233 \\
28 \\
48 \\
1 \\
127 \\
6,165 \\
37 \\
- \\
347 \\
28 \\
3,782 \\
1,972 \\
4,293 \\
35 \\
43 \\
2,359 \\
150 \\
916 \\
791\end{array}$ & $\begin{array}{r}239,921 \\
18,749 \\
2,965 \\
806 \\
11,526 \\
1,150 \\
1,914 \\
388 \\
88,152 \\
4,366 \\
20,315 \\
11,927 \\
10,268 \\
11,431 \\
29,846 \\
133,020 \\
75,601 \\
10,834 \\
13,776 \\
6,131 \\
11,780 \\
14,897\end{array}$ & $\begin{array}{r}5,386,675 \\
1,468,283 \\
376,785 \\
100,310 \\
863,872 \\
107,973 \\
6,248 \\
84 \\
84 \\
1,662,074 \\
86,185 \\
- \\
172,068 \\
106,238 \\
1,342,151 \\
145,432 \\
2,049,318 \\
1,973,541 \\
- \\
1,015 \\
74,762 \\
-\end{array}$ \\
\hline 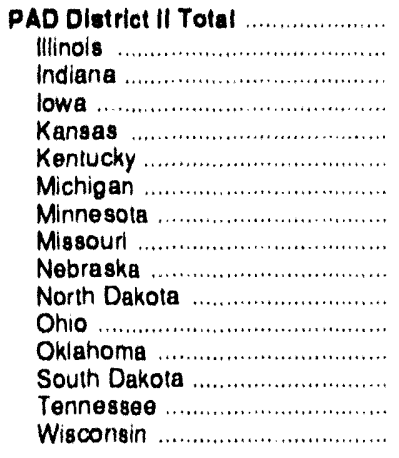 & $\begin{array}{r}1,689,057 \\
198,629 \\
81,586 \\
207,340 \\
161,105 \\
50,481 \\
84,034 \\
162,190 \\
73,730 \\
187,170 \\
100,635 \\
81,810 \\
89,645 \\
69,401 \\
48,291 \\
92,992\end{array}$ & $\begin{array}{r}151,670 \\
9,070 \\
8,814 \\
17,850 \\
2,143 \\
5,242 \\
13,990 \\
30,901 \\
735 \\
7,253 \\
7,319 \\
12,712 \\
5,300 \\
50 \\
30,342\end{array}$ & $\begin{array}{r}1,840,728 \\
207,699 \\
90,400 \\
225,190 \\
163,248 \\
55,733 \\
98,024 \\
193,081 \\
74,465 \\
194,423 \\
107,954 \\
94,530 \\
89,645 \\
74,701 \\
48,291 \\
123,334\end{array}$ & $\begin{array}{r}6,737 \\
298 \\
1,060 \\
669 \\
445 \\
575 \\
461 \\
297 \\
163 \\
309 \\
14 \\
1.715 \\
308 \\
144 \\
81 \\
200\end{array}$ & $\begin{array}{r}126,049 \\
19,636 \\
12,972 \\
3,312 \\
3,577 \\
13,363 \\
27,924 \\
4,091 \\
4,514 \\
674 \\
2,046 \\
18,186 \\
1,422 \\
2 \\
10,200 \\
3,130\end{array}$ & $\begin{array}{r}138,094 \\
94,468 \\
- \\
- \\
- \\
27,265 \\
15,734 \\
- \\
420 \\
207 \\
- \\
- \\
-\end{array}$ \\
\hline 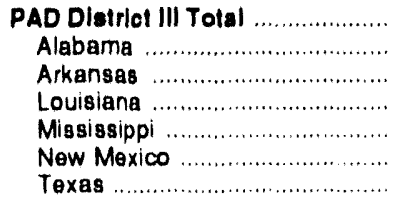 & $\begin{array}{r}546,274 \\
37,345 \\
85,982 \\
59,839 \\
80,931 \\
21,409 \\
260,767\end{array}$ & $\begin{array}{r}624 \\
371 \\
30 \\
- \\
14 \\
-\end{array}$ & $\begin{array}{r}546,898 \\
37,717 \\
86,011 \\
59,839 \\
80,945 \\
21,409 \\
260,976\end{array}$ & $\begin{array}{r}1,704 \\
41 \\
190 \\
205 \\
37 \\
15 \\
1,216\end{array}$ & $\begin{array}{r}40,461 \\
8,643 \\
7,774 \\
4,885 \\
1,154 \\
826 \\
17,180\end{array}$ & $\begin{array}{r}25,133 \\
743 \\
24,390 \\
-\end{array}$ \\
\hline 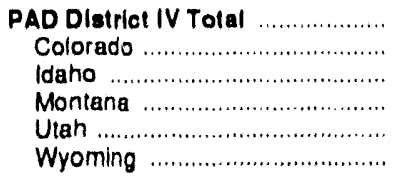 & $\begin{array}{r}198,728 \\
71,986 \\
59,716 \\
38,193 \\
14,685 \\
14,147\end{array}$ & $\begin{array}{r}5,879 \\
467 \\
3,491 \\
1,301 \\
278 \\
343\end{array}$ & $\begin{array}{r}204,607 \\
72,453 \\
63,207 \\
39,494 \\
14,963 \\
14,490\end{array}$ & $\begin{array}{r}265 \\
234 \\
11 \\
1 \\
13 \\
7\end{array}$ & $\begin{array}{r}6,483 \\
1,570 \\
58 \\
158 \\
1,676 \\
3,020\end{array}$ & $\begin{array}{l}- \\
\overline{-} \\
-\end{array}$ \\
\hline 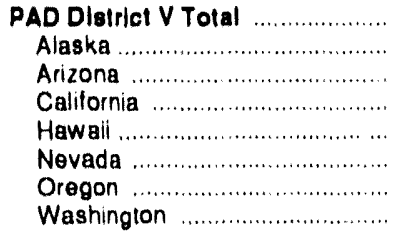 & $\begin{array}{r}456,462 \\
19,819 \\
318,702 \\
9,688 \\
5,283 \\
36,106 \\
66,865\end{array}$ & $\begin{array}{r}10,645 \\
1 \\
- \\
98 \\
1,801 \\
95 \\
6,745 \\
1,906\end{array}$ & $\begin{array}{r}467,108 \\
1 \\
19,819 \\
318,739 \\
11,489 \\
5,378 \\
42,851 \\
68,771\end{array}$ & $\begin{array}{r}384 \\
\overline{6} \\
233 \\
\overline{7} \\
51 \\
87\end{array}$ & $\begin{array}{r}128,770 \\
36,452 \\
3,193 \\
6,889 \\
79,953 \\
539 \\
1,207 \\
538\end{array}$ & $\begin{array}{r}431,540 \\
- \\
76 \\
413,179 \\
18,285 \\
-\end{array}$ \\
\hline
\end{tabular}

Dashes $(\cdot)$. No data reported.

Note: Totals may not equal sum of components due to independent rounding.

Sources: - Energy Intormation Administration Form ElA-821, "Annual Fuel OIl and Kerosene Sales Report." lor 1992

- Kerosene data are based on data from the Energy Intormation Administration's "Pelroleum Supply Annual," Volune 1, 1992, (DOE/EIA 034O(92)/1). 
Table 11. Sales for Transportation Use: Distlilate Fuel Oll and Residual Fuel Oll, 1992 (Thousand Gallons)

\begin{tabular}{|c|c|c|c|c|c|c|}
\hline \multirow{2}{*}{ Deatination } & \multirow{2}{*}{$\begin{array}{c}\text { Rallroad Use } \\
\begin{array}{c}\text { Dlatllinte } \\
\text { Fuel Oll }\end{array}\end{array}$} & \multicolumn{2}{|c|}{ Vessel Bunkering } & \multirow{2}{*}{$\begin{array}{c}\begin{array}{c}\text { On-Hlghway } \\
\text { Dienel }\end{array} \\
\begin{array}{c}\text { Dietlliate } \\
\text { Fuel Oll }\end{array}\end{array}$} & \multicolumn{2}{|c|}{ Total Transportation Use } \\
\hline & & $\begin{array}{l}\text { Dietillate } \\
\text { Fuel Oll }\end{array}$ & $\begin{array}{l}\text { Rosldual } \\
\text { Fual Oli }\end{array}$ & & $\begin{array}{l}\text { Distlliate } \\
\text { Fuel Oll }\end{array}$ & $\begin{array}{l}\text { Rouldual } \\
\text { Fuel Oll }\end{array}$ \\
\hline 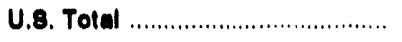 & $3,172,724$ & $2,218,692$ & $6,663,220$ & $21,374,872$ & $26,766,188$ & $6,863,220$ \\
\hline 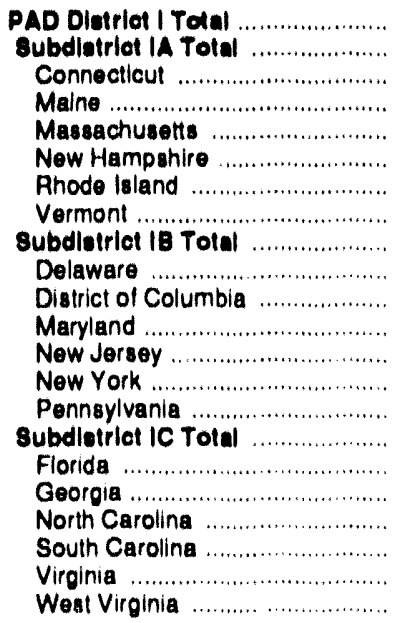 & $\begin{array}{r}601,734 \\
32,867 \\
10,052 \\
4,628 \\
12,913 \\
610 \\
842 \\
2, ; 20 \\
246,184 \\
1,303 \\
6,346 \\
29,497 \\
124,174 \\
35,069 \\
48,964 \\
323,813 \\
89,959 \\
65,351 \\
48,648 \\
16,008 \\
81,475 \\
22,372\end{array}$ & $\begin{array}{r}886,892 \\
88,619 \\
5,687 \\
13,847 \\
59,661 \\
370 \\
8,561 \\
393 \\
161,017 \\
3,916 \\
7 \\
11,887 \\
94,652 \\
19,676 \\
30,879 \\
307,356 \\
155,609 \\
35,278 \\
10,744 \\
8,453 \\
41,973 \\
55,298\end{array}$ & $\begin{array}{r}1,634,340 \\
14,768 \\
681 \\
71 \\
13,874 \\
140 \\
- \\
- \\
856,215 \\
38,427 \\
- \\
48,684 \\
366,755 \\
136,307 \\
268,043 \\
661,360 \\
399,141 \\
130,528 \\
14,735 \\
15,734 \\
101,221 \\
-\end{array}$ & $\begin{array}{r}6,688,163 \\
693,210 \\
185,287 \\
113,190 \\
247,409 \\
50,760 \\
37,344 \\
59,223 \\
2,569,627 \\
51,978 \\
20,881 \\
341,612 \\
373,082 \\
800,229 \\
981,845 \\
3,628,317 \\
893,762 \\
874,159 \\
661,167 \\
415,909 \\
586,726 \\
193,594\end{array}$ & $\begin{array}{r}8,046,789 \\
814,308 \\
201,927 \\
131,672 \\
319,984 \\
51,740 \\
46,747 \\
62,236 \\
2,976,998 \\
57,197 \\
27,234 \\
382,996 \\
591,909 \\
854,974 \\
1,061,689 \\
4,286,486 \\
1,139,330 \\
974,788 \\
720,559 \\
440,370 \\
710,174 \\
271,264\end{array}$ & $\begin{array}{r}1,634,340 \\
14,788 \\
681 \\
71 \\
13,874 \\
140 \\
- \\
-58 \\
868,215 \\
38,427 \\
-4 \\
48,684 \\
366,755 \\
136,307 \\
268,043 \\
661,360 \\
399,141 \\
130,528 \\
14,735 \\
15,734 \\
101,221\end{array}$ \\
\hline 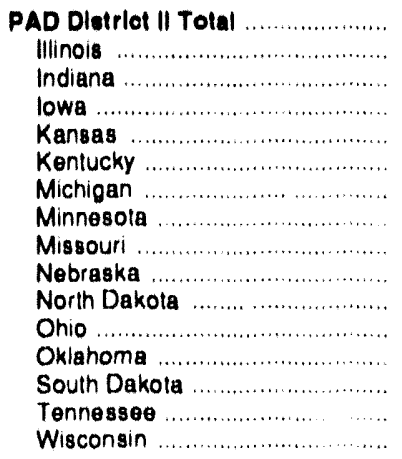 & $\begin{array}{r}1,242,347 \\
117,126 \\
192,713 \\
34,501 \\
145,012 \\
97,574 \\
32,927 \\
117,065 \\
16,294 \\
126,990 \\
38,901 \\
122,584 \\
101,745 \\
4,378 \\
71,814 \\
22,722\end{array}$ & $\begin{array}{r}440,494 \\
58,141 \\
13,578 \\
4,562 \\
- \\
139,600 \\
9,286 \\
4,870 \\
69,442 \\
- \\
- \\
14,387 \\
252 \\
- \\
123,277 \\
3,099\end{array}$ & $\begin{array}{r}17,968 \\
1,221 \\
8.041 \\
- \\
- \\
- \\
3,795 \\
100 \\
671 \\
- \\
\overline{3} \\
2,113 \\
- \\
1,713 \\
314\end{array}$ & $\begin{array}{r}7,093,942 \\
865,402 \\
753,113 \\
324,321 \\
258,207 \\
513,264 \\
556,507 \\
350,224 \\
644,586 \\
242,727 \\
97,445 \\
913,607 \\
390,810 \\
102,874 \\
596,009 \\
484,846\end{array}$ & $\begin{array}{r}8,776,782 \\
1,040,669 \\
959,404 \\
363,384 \\
403,219 \\
750,438 \\
598,720 \\
472,159 \\
730,322 \\
369,717 \\
136,346 \\
1,050,577 \\
492,807 \\
107,252 \\
791,100 \\
510,667\end{array}$ & $\begin{array}{r}17,968 \\
1,221 \\
8,041 \\
\\
3,785 \\
100 \\
671 \\
7 \\
2,113 \\
- \\
1.713 \\
314\end{array}$ \\
\hline 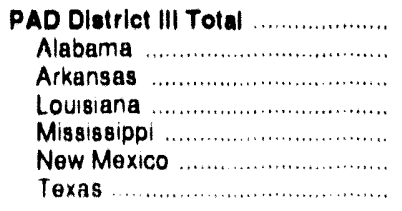 & $\begin{array}{r}667,178 \\
55,458 \\
28,607 \\
35,293 \\
24,532 \\
74,529 \\
448,760\end{array}$ & $\begin{array}{r}706,186 \\
73,199 \\
1,782 \\
323,747 \\
54,647 \\
- \\
252,781\end{array}$ & $\begin{array}{r}2,525,137 \\
136,732 \\
- \\
1,130,126 \\
101,225 \\
- \\
1,157,054\end{array}$ & $\begin{array}{r}3,446,063 \\
578,199 \\
399,995 \\
348,020 \\
317,122 \\
225,791 \\
1,575,936\end{array}$ & $\begin{array}{r}4,818,398 \\
706,857 \\
430,384 \\
707,059 \\
396,301 \\
300,320 \\
2,277,476\end{array}$ & $\begin{array}{r}2,625,137 \\
136,732 \\
1,130,126 \\
101,225 \\
1,157,054\end{array}$ \\
\hline $\begin{array}{l}\text { PAD District IV Total } \\
\text { Colorado } \\
\text { Idaho } \ldots \ldots \ldots \ldots \ldots \\
\text { Montana } \\
\text { Utah } \\
\text { Wyoming }\end{array}$ & $\begin{array}{r}254,897 \\
66,556 \\
20,093 \\
54,429 \\
40,704 \\
73,215\end{array}$ & $\begin{array}{r}112 \\
92 \\
20 \\
20\end{array}$ & $\begin{array}{l}- \\
- \\
- \\
- \\
-\end{array}$ & $\begin{array}{l}828,826 \\
215,220 \\
128,768 \\
132,587 \\
180,007 \\
172,244\end{array}$ & $\begin{array}{r}1,083,935 \\
281,776 \\
148,953 \\
187,016 \\
220,731 \\
245,459\end{array}$ & - \\
\hline 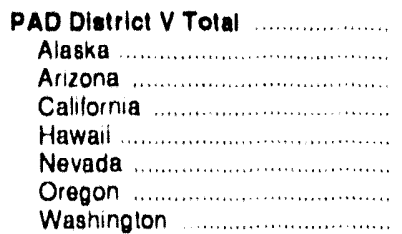 & $\begin{array}{r}406,468 \\
5,296 \\
7,120 \\
217,250 \\
22 \\
4,164 \\
114,668 \\
57,947\end{array}$ & $\begin{array}{r}514,938 \\
153,274 \\
23 \\
111,884 \\
89,697 \\
- \\
50,248 \\
109,811\end{array}$ & $\begin{array}{r}2,485,775 \\
12,236 \\
- \\
1,247,458 \\
138,828 \\
- \\
225,152 \\
862,102\end{array}$ & $\begin{array}{r}3,118,878 \\
49,225 \\
351,527 \\
1,842,723 \\
24,850 \\
152,457 \\
329,762 \\
368,334\end{array}$ & $\begin{array}{r}4,040,284 \\
207,795 \\
358,671 \\
2,171,857 \\
114,569 \\
156,621 \\
494,678 \\
536,093\end{array}$ & $\begin{array}{r}2,486,775 \\
12,236 \\
1,247,458 \\
138,828 \\
225,152 \\
862,102\end{array}$ \\
\hline
\end{tabular}

Dashes $(\cdot)$ - No data reported

Note: Totals may not equal sum of components due to independent rounding.

Sources: "Energy Intormation Administration Form ElA 821, "Annual Fuel Oil and Kerosene Sales Report," for 1992.

- On. Highiway Diesel data are Federal Highway Administation statistics of highway special fuels use. 
Table 12. Sales for Military, Off-Hlghway, and All Other Uses: Distlliate Fuel Oll, Residual Fuel Oll, and Kerosene, 1992

(Thousand Gallons)

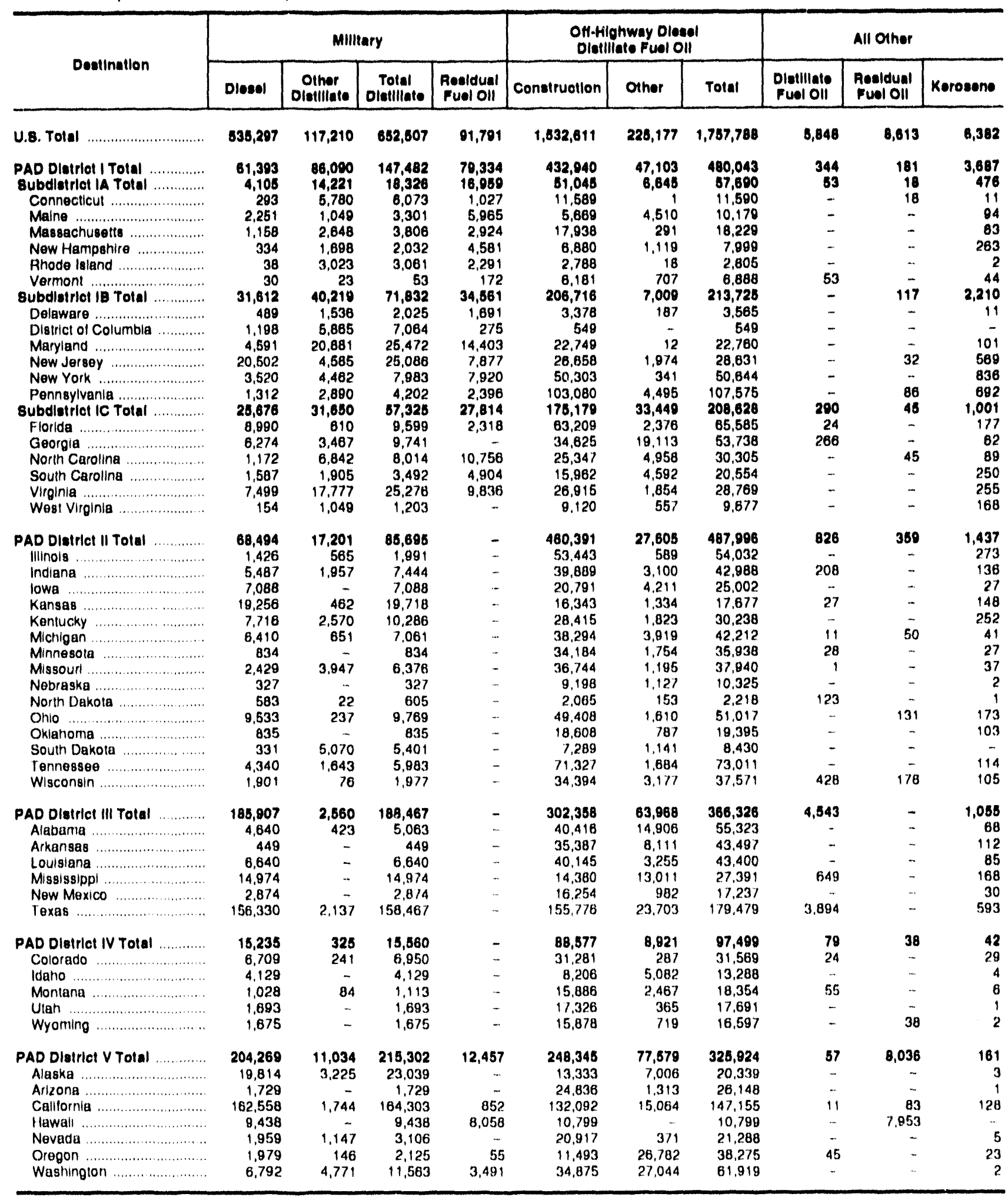

Dashes $(\cdot)=$ No data reported

Note: Totals may not equal sum of components due to independent rounding.

Sources: - Enorgy informatlon Administration Form ElA-821. "Annual Fuel Oil and Kerosene Sales Heport," for 199?

- Kerosene data are based on data trom the Energy Information Administration's "Pelroleum Supply Annual," Volume 1, 1992, (DOE/EIA-0340(92)/1) 


\section{Adjusted Sales of Fuel Oil and Kerosene in 1992}

The following tables contain estimates of distillate fuel oil that have been adjusted at the PAI) district level to equal published EIA volume estimates of petroleum products supplied in the U.S. marketplace. 'The kerosene and residual fuel oil sales estimates have beon adjusted at the national level. Ilistorically, kerosene estimates have always been adjusted at the PAD district level. However, in 1992, certain kerosene estimates at the $\mathrm{PAD}$ district level resulted in negative numbers. Hence, the adjustment procedure was changed to display sales volumes of kerosene for all PAD districts.
The products supplied estimates can be found in the 1988 through 1992 Petroleum Supply Anmual. For certain sales categories, data obtained from ulternute sourees are used instend of the ndjusted numbers. See "Technical Note 3 " in Appendix for further explamation of data adjustments.

Total domestic adjusted sules of fuel oil and kerosene declined slightly from 63.3.3 billion gallons in 1991 to 63.2 billions gullons in 1992, While ndjusted sules of distillute increased 2.3 percent in 1992 , rosidual fuol oil dropped 5.3 perent. Kerosene adjusted sales declined by 10.3 pereent.

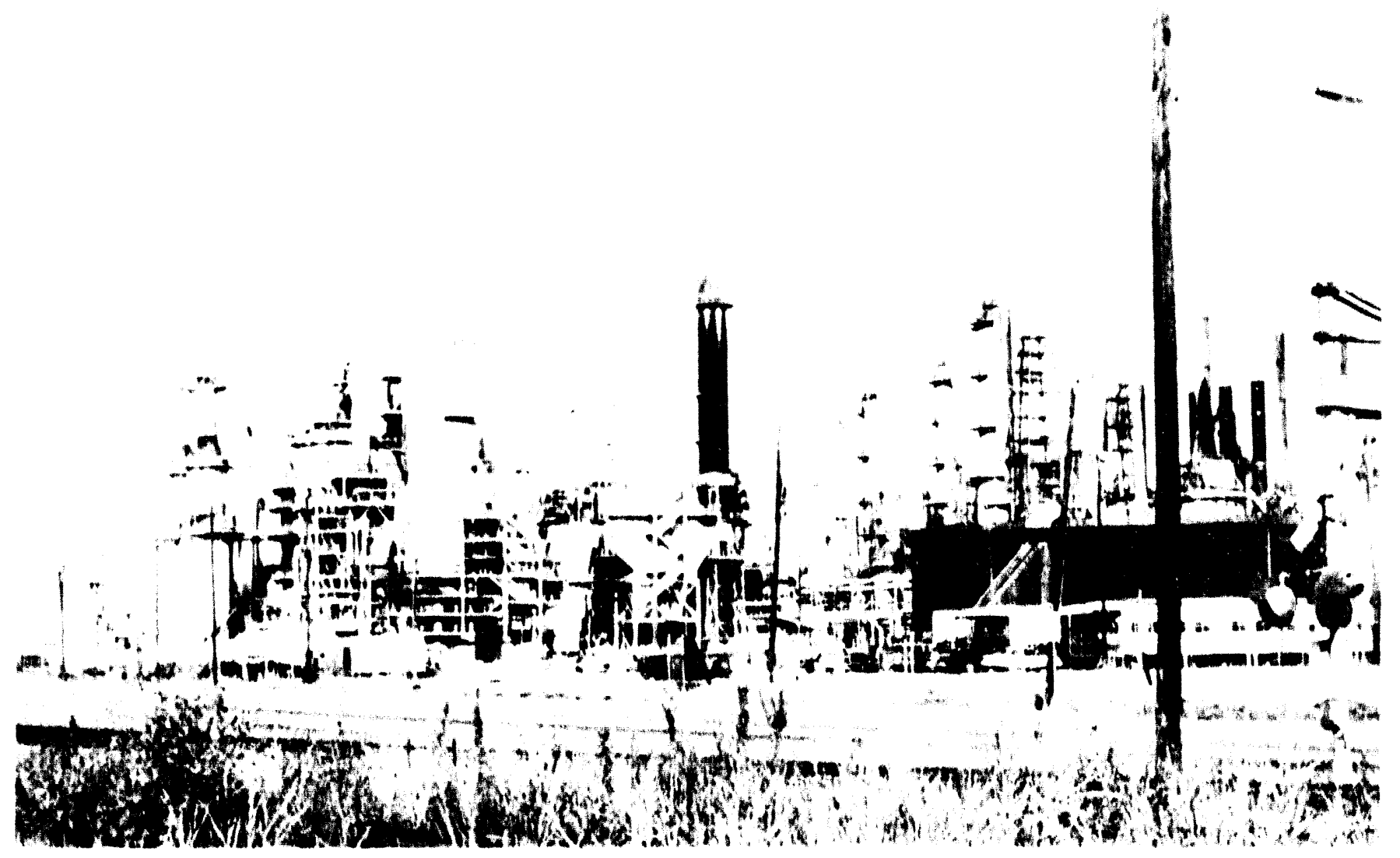

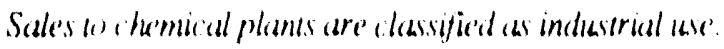


Table 13. Adjusted Sales of Dlstillate Fuel Oll by End Use in the Unlted States: 1988-1992 (Thousand Gallons)

\begin{tabular}{|c|c|c|c|c|c|}
\hline \multirow{2}{*}{ End Use } & \multicolumn{5}{|c|}{ Diatlilate Fuol Oli } \\
\hline & 1988 & 1988 & 1900 & 1091 & 1002 \\
\hline 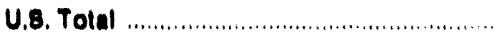 & $47,088,420$ & $48,393,480$ & $46,308,120$ & $44,776,360$ & $48,791,408$ \\
\hline 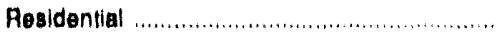 & $7,662,046$ & $7.501,098$ & $6,022,902$ & $5,993,482$ & $6,238,133$ \\
\hline 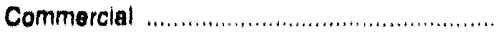 & $4,136,079$ & $3,858,743$ & $3,502,778$ & $3,471,468$ & $3,347,133$ \\
\hline 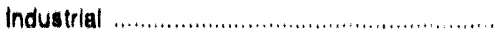 & $2,617,639$ & $2,585,283$ & $2,452,322$ & $2,320,518$ & $2,218,843$ \\
\hline 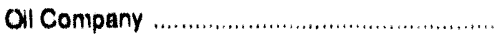 & 872,133 & 645,217 & 968,764 & $9: 0,781$ & 785,120 \\
\hline Farm & $3,420,671$ & $3,196,928$ & $3,293,782$ & $3,285,639$ & $3,504,846$ \\
\hline 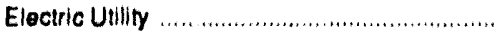 & 800,085 & $1,078,887$ & 733,681 & 590.801 & 468,795 \\
\hline 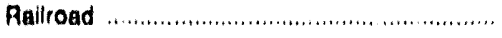 & $3,259,250$ & $3,264,229$ & $3,210,111$ & $3,028,292$ & $3,217,231$ \\
\hline 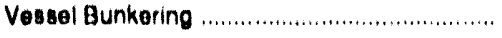 & $2,310,367$ & $2,356,444$ & $2,197,004$ & $2,167,640$ & $2,240,170$ \\
\hline 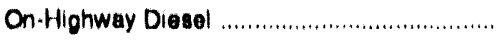 & $20,038,691$ & $21,131,692$ & $21,300,477$ & $20,485,364$ & $21,374,872$ \\
\hline 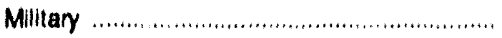 & 980,877 & 928,316 & 784,401 & 828.733 & 650,178 \\
\hline 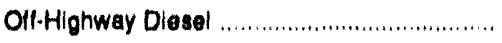 & $1,821,618$ & $1,637,497$ & $1,776,352$ & $1,686,262$ & $1,739,068$ \\
\hline 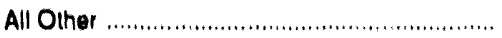 & 66,763 & 29,108 & 2.541 & 2,399 & 7,075 \\
\hline
\end{tabular}

Note: Totals may not equal sum ol components due to independent rounding.

Note: Distillate tuel oil date in the Adjusted Sales tables (13.24) are adjusted at the PAD District level. See Tochnical Nole 3 lor lurther explanation

Sources: - Eneroy Intormation Administration Form ElA.821, "Annual Fuel Oil and Kerosene Sales Report," lor 1988.1992.

- Electric Uillity data are based on data obtained on Form EiA 759, "Monthly Power Plant Roport," and F orm FEnC.423, "Monthly Report ol Cost and Quality of Fuels lor Electric Plante."

- On Highway Diesel deta are Fedoral Highway Administation statistics ol highway special huols use

- Energy Intoimation Administration's "Potroleum Supply Annual," Volume 1, 1888.1892, (DOE/EIA 0340(88)/1 - (82)/1)

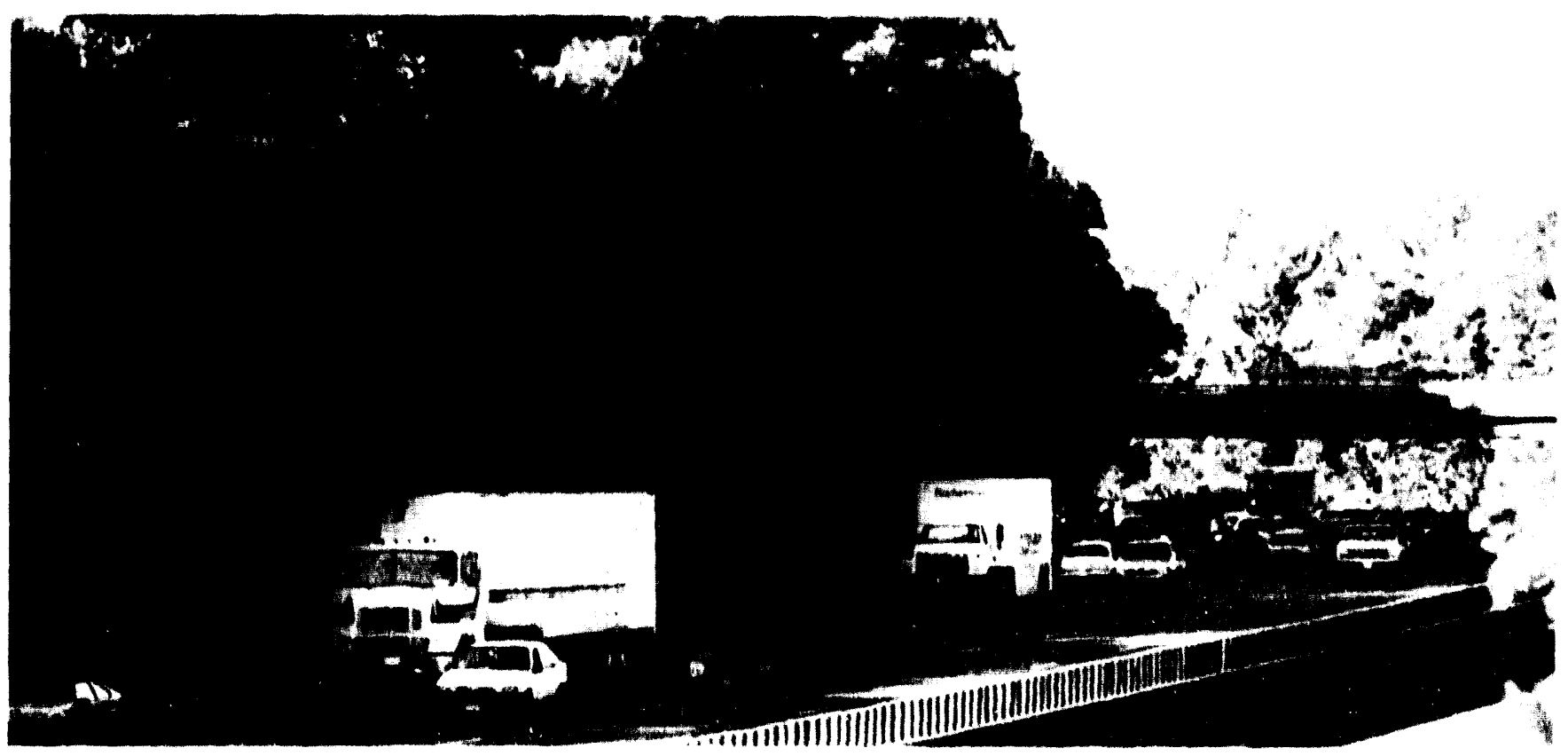

More diesel is used for on-highway use than for any other ust'. 
Table 14. Adjusted Sales of Resldual Fuel Oll by End Use In the Unlted States: 1988-1992 (Thousand Gallons)

\begin{tabular}{|c|c|c|c|c|c|}
\hline \multirow{2}{*}{ End Uee } & \multicolumn{5}{|c|}{ Aealdual Fuol oll } \\
\hline & 1088 & 1089 & 1000 & 1001 & 1092 \\
\hline 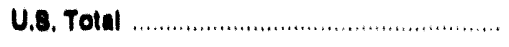 & $21,179,860$ & $21,002,814$ & $18,837,882$ & $17,780,260$ & $16,616,674$ \\
\hline 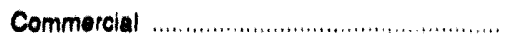 & $1,827,715$ & $1,650,418$ & $1,503,301$ & $1,426,658$ & $1,289,987$ \\
\hline 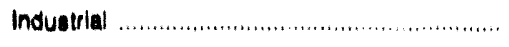 & $3,071,782$ & $2, \$ 53,978$ & $2,227,855$ & $1,038,473$ & $2,332,811$ \\
\hline Oil Company & 520,516 & 344,751 & 314,358 & 301.511 & 297,332 \\
\hline 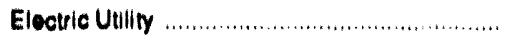 & $9,540,878$ & $9,797,006$ & $8,433,480$ & $7,169,509$ & $6,587,236$ \\
\hline 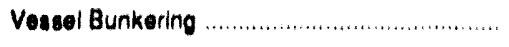 & $6,025,511$ & $0,621,100$ & $6,248,005$ & $0,780.056$ & $7,199,078$ \\
\hline 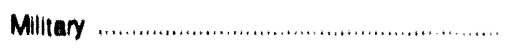 & 137,207 & 105.255 & 81,992 & 120.204 & 100,684 \\
\hline 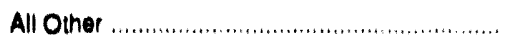 & 55,862 & 29,348 & 28,791 & $i .752$ & 0,448 \\
\hline
\end{tabular}

- Estimated data. Annual estimate is based on eleven months of data

Note: Totals may not equal sum of components due to independent rounding

Note: Residual luel oil data in the Adjusted Sales tables (13-24) are adjusted at the national level See Technical Nole 3 lor lurther explanation

Sources: - Energy Inlormation Administration Form E1A.821, "Annual Fuel Oil and Kerosene Sales Report," lor 1988.1992.

- Electric Utility duta are based on data obtained on Form ElA.759, "Monthly Powar Plant Report," and Form FERC.423, "Monthly Report ol Cost and Ouality of Fuels lor Electric Plante."

- For 1088 through 1090, military data lo residual fuel oil are based on the Delense Fuel Supply Center's "DEIS Report" FiA 821 survey results are used lor 1001 and 1002

- Energy Intormation Administration's "Potroloum Supply Annual," Volume 1, 1089-1902, (DOE/ElA 0340(88)/1 - (82)/1)

Table 15. Adjusted Sales of Kerosene by End Use In the Unlted States: $1988-1992$ (Thousand Gallons)

\begin{tabular}{|c|c|c|c|c|c|}
\hline \multirow{2}{*}{ End Uee } & \multicolumn{5}{|c|}{ Korosene } \\
\hline & 1088 & 1080 & 1990 & 1001 & 1092 \\
\hline U.8. Tolal & $1,478,316$ & $1,291,688$ & 682,008 & 708,716 & 636,428 \\
\hline (מ, & 770,042 & 618.322 & 439,870 & 491,029 & 481,511 \\
\hline 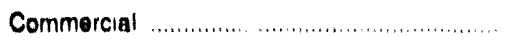 & 308,584 & 349,560 & 05,737 & 97,186 & 82,308 \\
\hline 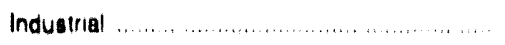 & 188,117 & 171.124 & 76,687 & 76,055 & 46,000 \\
\hline 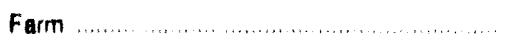 & 45,990 & 30,901 & 21,934 & 24,668 & 20,036 \\
\hline All Other .............. & 167,583 & 115,150 & 17,180 & 10,678 & 6.382 \\
\hline
\end{tabular}

Note: Totals may not equal sum of componenis duo 10 independent rounding

Nole. Kerosene data in the Adjusted Sales tables $(1324)$ are adjusted at the national level for 1092 and at the PAD district level tor prior years. See Technical Note 3 lor further explanation.

Sources: - Kerosene date are based on data from the Eneroy Intormation Administration's "Potroleum Supply Annual," Volume 1, 1989 1982, (DOE/EIA $0340(88) / 1 \cdot(92) / 1)$ 
Table 16. Adjusted Sales of Distillate Fuel OII by End Use, 1991 and 1992 (Thousand Gallons)

\begin{tabular}{|c|c|c|c|c|c|c|}
\hline \multirow{2}{*}{ Destination } & \multicolumn{2}{|c|}{ Rooldentlal } & \multicolumn{2}{|c|}{ Commerelal } & \multicolumn{2}{|c|}{ Induotrial } \\
\hline & 1001 & 1902 & 1901 & 1002 & 1001 & 1002 \\
\hline U.e. Total & $3,003,402$ & $0,238,133$ & $3,471,498$ & $3,347,133$ & $2,320,810$ & $2,210,843$ \\
\hline 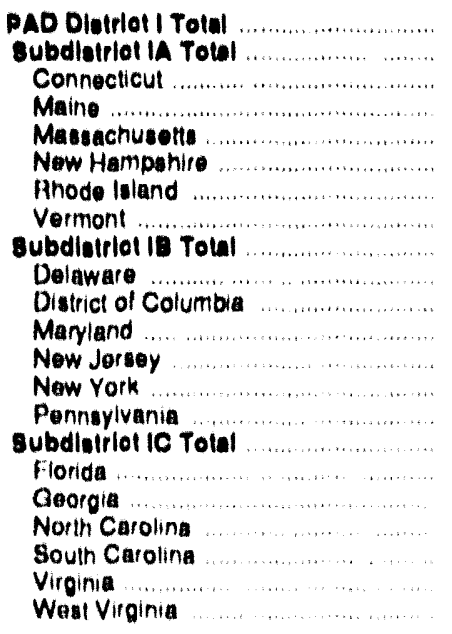 & $\begin{array}{r}4,616,029 \\
1,734,076 \\
471,740 \\
216,820 \\
698,863 \\
149,708 \\
112,864 \\
95,490 \\
2,474,466 \\
42,689 \\
6,931 \\
175,837 \\
464,744 \\
1,050,560 \\
734,006 \\
409,181 \\
0,068 \\
7,494 \\
134,410 \\
41,005 \\
192,841 \\
22,663\end{array}$ & $\begin{array}{r}8,004,006 \\
1,900,947 \\
384,448 \\
221,918 \\
790,410 \\
184,726 \\
137,407 \\
92,044 \\
2,020,219 \\
43,745 \\
7,137 \\
187,286 \\
470,600 \\
1,176,304 \\
741,148 \\
418,940 \\
12,997 \\
7,460 \\
111,110 \\
26,978 \\
200,862 \\
19,414\end{array}$ & $\begin{array}{r}2,168,611 \\
818,208 \\
128,297 \\
60,634 \\
310,517 \\
47,684 \\
32,567 \\
29,389 \\
1,179,423 \\
18,470 \\
24,653 \\
96,454 \\
275,378 \\
536,644 \\
228,824 \\
371,039 \\
125,017 \\
36,208 \\
76,468 \\
21,940 \\
89,530 \\
21,709\end{array}$ & $\begin{array}{r}2,170,731 \\
803,038 \\
123,672 \\
72,067 \\
280,878 \\
47,134 \\
25,316 \\
34,272 \\
1,224,017 \\
14,650 \\
23,147 \\
108,199 \\
267,377 \\
583,082 \\
227,263 \\
382,470 \\
126,150 \\
43,611 \\
68,683 \\
28,192 \\
82,131 \\
13,511\end{array}$ & $\begin{array}{r}841,407 \\
94,064 \\
28,132 \\
16,682 \\
27,164 \\
7,717 \\
6,203 \\
7,167 \\
240,339 \\
6,066 \\
0 \\
26,094 \\
92,181 \\
45,537 \\
109,552 \\
308,183 \\
33,288 \\
45,657 \\
56,560 \\
27,713 \\
50,823 \\
80,944\end{array}$ & 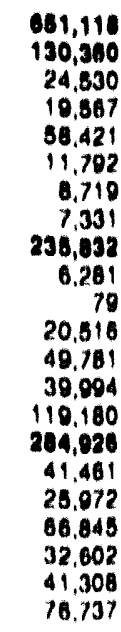 \\
\hline 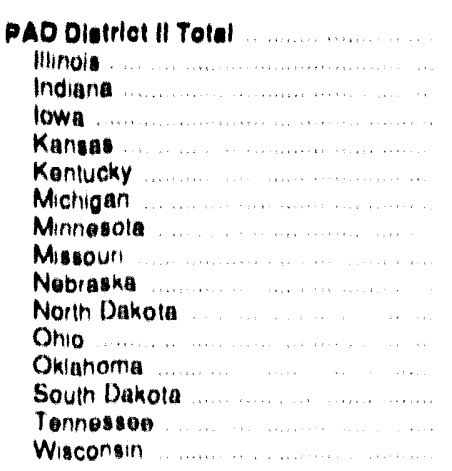 & $\begin{array}{r}1,006,709 \\
51,542 \\
81,346 \\
37,232 \\
982 \\
29,325 \\
191,368 \\
172,048 \\
18,040 \\
8,288 \\
37,871 \\
17,219 \\
3 \\
33,741 \\
11,268 \\
215,307\end{array}$ & 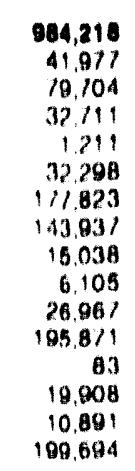 & $\begin{array}{r}973,600 \\
70,034 \\
49.384 \\
23,652 \\
15,235 \\
30,046 \\
81,376 \\
38,214 \\
46,648 \\
7,682 \\
6,705 \\
61,818 \\
20,368 \\
8,045 \\
25,212 \\
82,28 ?\end{array}$ & $\begin{array}{r}692,778 \\
78,688 \\
59,467 \\
20,508 \\
21,074 \\
36.693 \\
74,258 \\
31,914 \\
49.343 \\
11,338 \\
6,613 \\
70,707 \\
18,726 \\
10,310 \\
43,765 \\
65,177\end{array}$ & $\begin{array}{r}673,400 \\
62,841 \\
91,858 \\
15,944 \\
7,873 \\
153,604 \\
52,858 \\
38,423 \\
27,988 \\
4,108 \\
17,680 \\
90,208 \\
24,694 \\
5,348 \\
32,506 \\
18,384\end{array}$ & $\begin{array}{r}714,020 \\
90,409 \\
98,805 \\
16,868 \\
9,805 \\
136,845 \\
51,140 \\
38,606 \\
26,120 \\
6,470 \\
10,116 \\
121,632 \\
16,106 \\
10,408 \\
34,204 \\
17,491\end{array}$ \\
\hline $\begin{array}{l}\text { PAD Distrlet lil Tolal } \\
\text { Alabama } \\
\text { Alkansas } \\
\text { lovisiana } \\
\text { Mississipps } \\
\text { Now Moxico } \\
\text { loxas }\end{array}$ & $\begin{array}{r}1,444 \\
161 \\
33 \\
10 \\
73 \\
389 \\
138\end{array}$ & $\begin{array}{r}1,730 \\
449 \\
527 \\
3 \\
5.3 \\
60 ? \\
05\end{array}$ & $\begin{array}{r}262,002 \\
41,249 \\
14,357 \\
37,727 \\
25,503 \\
10.402 \\
127,854\end{array}$ & $\begin{array}{r}246,647 \\
43,271 \\
15,871 \\
25,445 \\
21,453 \\
10,109 \\
130.395\end{array}$ & $\begin{array}{r}388.071 \\
114.081 \\
16.007 \\
29.369 \\
19.703 \\
34.100 \\
155.411\end{array}$ & $\begin{array}{r}386,280 \\
96,222 \\
14,617 \\
49,720 \\
30,870 \\
16,972 \\
147,881\end{array}$ \\
\hline $\begin{array}{l}\text { PAD Distrlet IV Total } \\
\text { Colorado } \\
\text { Idano } \\
\text { Montana } \\
\text { Utah } \\
\text { Wyoming }\end{array}$ & $\begin{array}{r}56,038 \\
1,132 \\
20,538 \\
14,944 \\
6,775 \\
3,648\end{array}$ & $\begin{array}{r}41,301 \\
905 \\
23,947 \\
9,145 \\
4,647 \\
2,456\end{array}$ & $\begin{array}{r}81,347 \\
24,798 \\
18,211 \\
6,545 \\
10,702 \\
10091\end{array}$ & $\begin{array}{r}98,021 \\
35,040 \\
17,412 \\
1,111 \\
19,742 \\
9.316\end{array}$ & $\begin{array}{r}248,096 \\
47,397 \\
30,371 \\
57,083 \\
42,378 \\
59,601\end{array}$ & $\begin{array}{r}193,086 \\
51,091 \\
13,556 \\
39,858 \\
33,360 \\
55,194\end{array}$ \\
\hline $\begin{array}{l}\text { PAD oletrlet V Total } \\
\text { Alaska } \\
\text { Anzona } \\
\text { Culitomina } \\
\text { Hawail } \\
\text { Nevada } \\
\text { Orogon } \\
\text { Washirigton }\end{array}$ & $\begin{array}{r}281,590 \\
67,043 \\
213 \\
8,359 \\
16 \\
9,291 \\
62,454 \\
104,214\end{array}$ & $\begin{array}{r}208,079 \\
67,459 \\
220 \\
8,475 \\
12 \\
9,109 \\
44,615 \\
76,179\end{array}$ & $\begin{array}{r}387,867 \\
40,911 \\
12,127 \\
186,788 \\
25.12 ? \\
12,330 \\
41,757 \\
67,62 ?\end{array}$ & $\begin{array}{r}248,458 \\
57,830 \\
9,463 \\
83,787 \\
18,351 \\
12,498 \\
32,233 \\
34,275\end{array}$ & $\begin{array}{r}397,126 \\
10,273 \\
54,170 \\
169,924 \\
4,482 \\
103,220 \\
19,538 \\
35,518\end{array}$ & $\begin{array}{r}304,361 \\
17,750 \\
61,644 \\
58,191 \\
5,327 \\
103,187 \\
26,401 \\
31,861\end{array}$ \\
\hline
\end{tabular}

Sese loolnoles at end of lable 
Table 16. Adjusted Sales of Diatlliate Fuel Oll by End Use, 1991 and 1992 (Continued) (Thousand Gallons)

\begin{tabular}{|c|c|c|c|c|c|c|}
\hline \multirow{2}{*}{ Destination } & \multicolumn{2}{|c|}{ Oll Company } & \multicolumn{2}{|c|}{ Farm } & \multicolumn{2}{|c|}{ Eleotrle Utility } \\
\hline & 1001 & 1902 & 1001 & 1002 & 1001 & 1002 \\
\hline 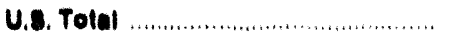 & 010,701 & 706,120 & $3,208,030$ & $3,804,040$ & 800,001 & 408,708 \\
\hline 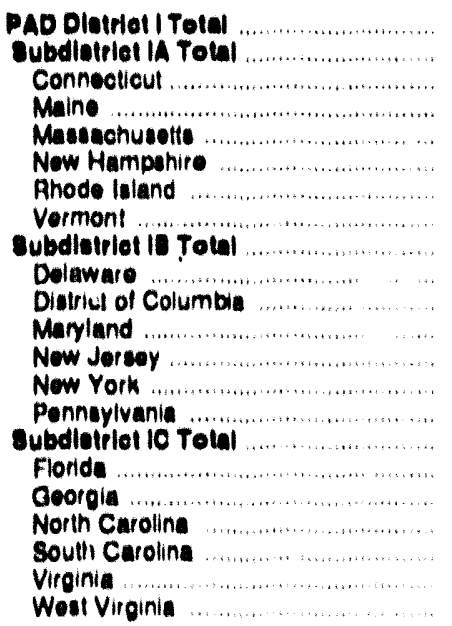 & $\begin{array}{r}20,100 \\
3,028 \\
100 \\
2.841 \\
396 \\
54 \\
218 \\
17 \\
10,613 \\
203 \\
764 \\
2,332 \\
688 \\
6,437 \\
6,781 \\
1,203 \\
374 \\
98 \\
384 \\
697 \\
2,003\end{array}$ & $\begin{array}{r}13.070 \\
1,007 \\
336 \\
96 \\
344 \\
69 \\
2.42 \\
19 \\
7,094 \\
286 \\
681 \\
2.871 \\
823 \\
3.983 \\
4,008 \\
1.023 \\
242 \\
120 \\
162 \\
875 \\
1,912\end{array}$ & 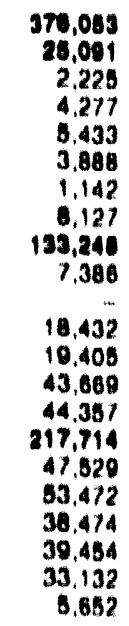 & 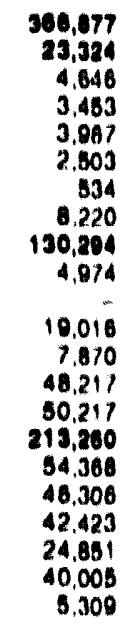 & 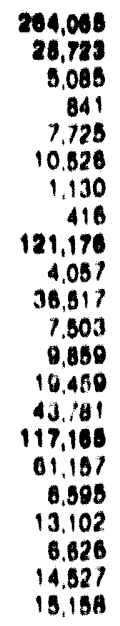 & 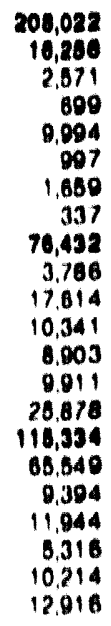 \\
\hline 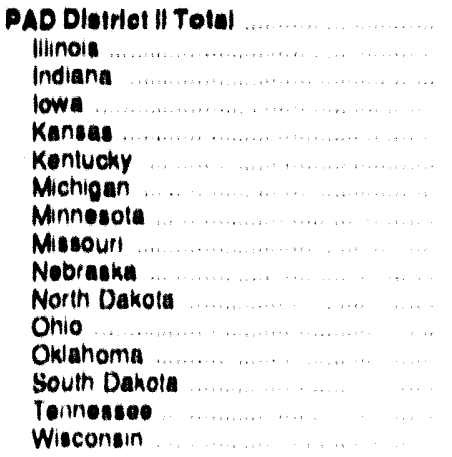 & $\begin{array}{r}03,443 \\
1.242 \\
1.283 \\
146 \\
11,682 \\
1,073 \\
1,365 \\
128 \\
411 \\
10 \\
5,861 \\
3,688 \\
34,560 \\
19 \\
689 \\
636\end{array}$ & $\begin{array}{r}1,800 \\
1,680 \\
1,330 \\
182 \\
4,734 \\
2.511 \\
6.338 \\
858 \\
300 \\
28 \\
5.688 \\
4.010 \\
51.268 \\
19 \\
1.096 \\
594\end{array}$ & 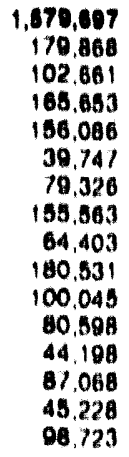 & 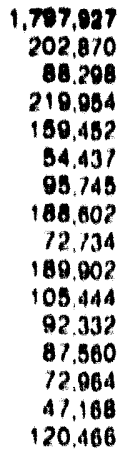 & $\begin{array}{r}142,332 \\
21,377 \\
18,178 \\
2,008 \\
4,116 \\
11,059 \\
20,115 \\
4,768 \\
0,415 \\
1,056 \\
2,220 \\
30,162 \\
428 \\
710 \\
7,302 \\
10,760\end{array}$ & $\begin{array}{r}109,422 \\
11,025 \\
11,248 \\
2,872 \\
3,101 \\
11,067 \\
24,211 \\
3,647 \\
3,014 \\
584 \\
1,174 \\
15,768 \\
1,233 \\
1 \\
8,844 \\
2,114\end{array}$ \\
\hline 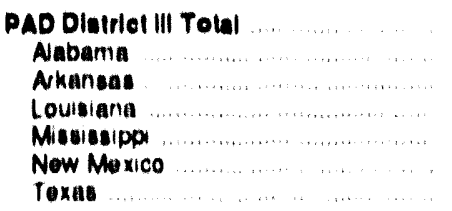 & $\begin{array}{r}720.728 \\
14.263 \\
3.632 \\
355.176 \\
6.054 \\
16.098 \\
326.605\end{array}$ & $\begin{array}{r}884.408 \\
10.851 \\
3.312 \\
206.086 \\
11.001 \\
13.831 \\
249.111\end{array}$ & $\begin{array}{r}699,746 \\
44,956 \\
67,660 \\
89.668 \\
151,040 \\
17,388 \\
290,148\end{array}$ & $\begin{array}{r}703,240 \\
48,490 \\
110,400 \\
76,940 \\
104,084 \\
27,530 \\
335,58 ?\end{array}$ & $\begin{array}{r}46,711 \\
8,048 \\
8,437 \\
3,827 \\
3.667 \\
5,108 \\
15.614\end{array}$ & $\begin{array}{r}36,001 \\
7,403 \\
6,740 \\
4,238 \\
1,000 \\
717 \\
14,090\end{array}$ \\
\hline 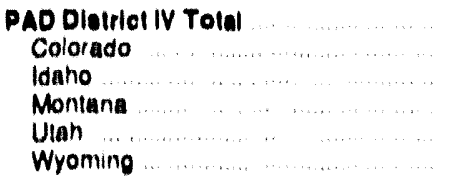 & $\begin{array}{r}24,448 \\
3,803 \\
76 \\
3,573 \\
4,482 \\
12.515\end{array}$ & $\begin{array}{r}43,616 \\
9.694 \\
62 \\
1.06 / \\
10,165 \\
22.501\end{array}$ & $\begin{array}{r}247,026 \\
54,500 \\
92,581 \\
11,826 \\
12,641 \\
15,417\end{array}$ & $\begin{array}{r}239,043 \\
84,930 \\
74,09 ? \\
40,296 \\
17,540 \\
16,096\end{array}$ & $\begin{array}{r}9,857 \\
1,119 \\
462 \\
1,056 \\
2.319 \\
4,301\end{array}$ & $\begin{array}{r}8,621 \\
1.36 ! \\
5 ! \\
137 \\
1.453 \\
2.619\end{array}$ \\
\hline 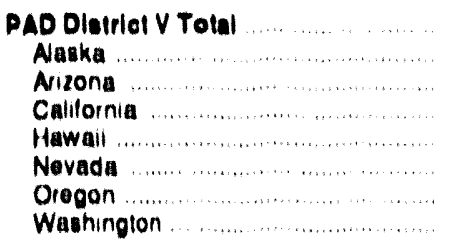 & $\begin{array}{r}1,061 \\
58,027 \\
308 \\
11,136 \\
1,169 \\
237 \\
267 \\
10,007\end{array}$ & $\begin{array}{r}61,632 \\
47,865 \\
173 \\
12.248 \\
1,043 \\
189 \\
3 \\
11 ?\end{array}$ & $\begin{array}{r}413,110 \\
17,292 \\
269,901 \\
9,634 \\
8,565 \\
42,282 \\
68,444\end{array}$ & $\begin{array}{r}308,989 \\
1 \\
16,842 \\
270,922 \\
9,164 \\
4,610 \\
36,416 \\
58,443\end{array}$ & $\begin{array}{r}129,037 \\
25,589 \\
2,192 \\
11,532 \\
74,626 \\
5,405 \\
3,583 \\
5,310\end{array}$ & $\begin{array}{r}111,649 \\
31,605 \\
2,768 \\
6,973 \\
69,922 \\
467 \\
1.046 \\
467\end{array}$ \\
\hline
\end{tabular}

Seo lootnoles at and of table 
Table 16. Adjuated 8ales of Dlatllate Fuel Oll by End Use, 1991 and 1992 (Continued) (Thousand Gallons)

\begin{tabular}{|c|c|c|c|c|c|c|}
\hline \multirow{2}{*}{ Desilination } & \multicolumn{2}{|c|}{ Rulitroud } & \multicolumn{2}{|c|}{ Veooul Bunkering } & \multicolumn{2}{|c|}{ On.Hlghway } \\
\hline & 1801 & 1002 & 1001 & $10 \% 2$ & 1091 & 1002 \\
\hline U.8. Told & $3,020,202$ & $3,217,231$ & $2,107,040$ & $2,240,170$ & $20,408,364$ & $21,374,072$ \\
\hline 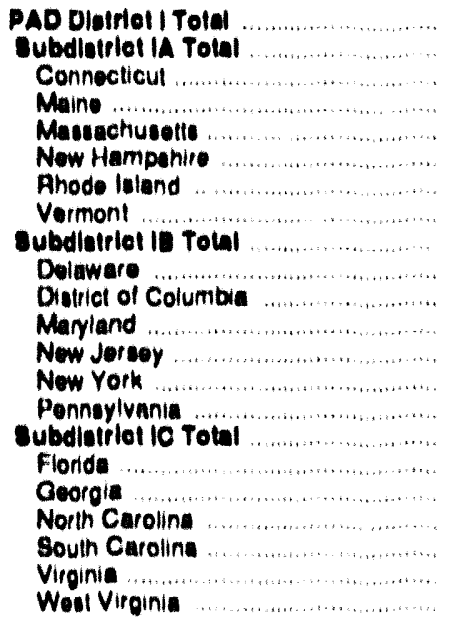 & 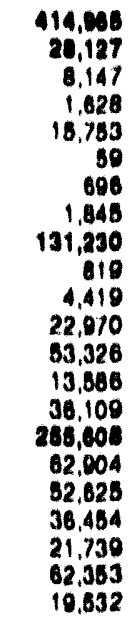 & $\begin{array}{r}801,031 \\
27,144 \\
9,128 \\
3,858 \\
10,763 \\
809 \\
102 \\
2,184 \\
204,487 \\
1,086 \\
8,289 \\
24,868 \\
103,496 \\
29,230 \\
40,810 \\
208,090 \\
74,079 \\
\$ 4,468 \\
40,547 \\
13,342 \\
67,007 \\
18,647\end{array}$ & $\begin{array}{r}411,007 \\
66,088 \\
2,048 \\
13,689 \\
11,212 \\
184 \\
7,628 \\
340 \\
131,080 \\
8,038 \\
5 \\
7,789 \\
79,398 \\
11,749 \\
26,807 \\
214,089 \\
90,730 \\
19,099 \\
12,801 \\
4,640 \\
38,027 \\
43,887\end{array}$ & $\begin{array}{r}464,186 \\
73,770 \\
4,740 \\
11,641 \\
40,726 \\
308 \\
7,136 \\
327 \\
134,204 \\
3,264 \\
6 \\
9,808 \\
78,890 \\
18,390 \\
28,737 \\
286,174 \\
129,697 \\
20,404 \\
9,056 \\
7,046 \\
34,084 \\
48,000\end{array}$ & 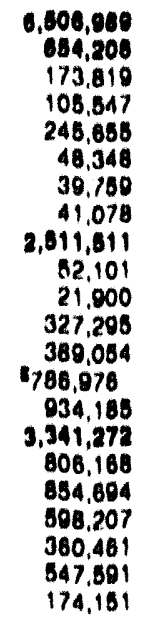 & 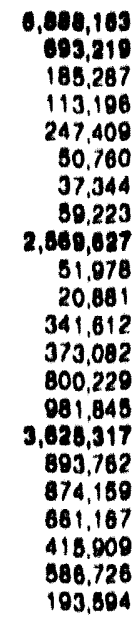 \\
\hline 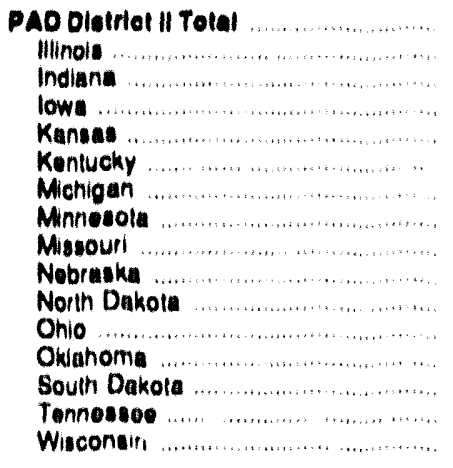 & $\begin{array}{r}1,179,770 \\
119,177 \\
102,300 \\
20,638 \\
166,464 \\
100,238 \\
34,024 \\
67,677 \\
24,227 \\
111,478 \\
39,462 \\
96,200 \\
84,860 \\
4,510 \\
70,077 \\
31,622\end{array}$ & $\begin{array}{r}1,213,480 \\
111,402 \\
188,232 \\
31,690 \\
141,640 \\
98,306 \\
32,162 \\
114,343 \\
16,919 \\
124,038 \\
31,097 \\
119,733 \\
90,380 \\
1,276 \\
70,144 \\
22,194\end{array}$ & $\begin{array}{r}411,020 \\
84,483 \\
0,000 \\
4,581 \\
138,424 \\
4,468 \\
3,277 \\
39,740 \\
14,530 \\
179,601 \\
3,054\end{array}$ & $\begin{array}{r}490,281 \\
86,780 \\
13,282 \\
4,486 \\
136,364 \\
9,070 \\
4.767 \\
67,827 \\
14,052 \\
246 \\
120,410 \\
3,027\end{array}$ & $\begin{array}{r}0,030,100 \\
677,536 \\
784,030 \\
319,894 \\
276,312 \\
417,619 \\
531,300 \\
369,176 \\
584,823 \\
220,319 \\
94,596 \\
886,816 \\
352,086 \\
90,480 \\
575,503 \\
418,663\end{array}$ & $\begin{array}{r}7,093,042 \\
889,402 \\
783,113 \\
324,321 \\
288,207 \\
313,264 \\
586,607 \\
350,224 \\
644,886 \\
242,127 \\
97,445 \\
913,607 \\
390,810 \\
102,874 \\
596,009 \\
484,646\end{array}$ \\
\hline 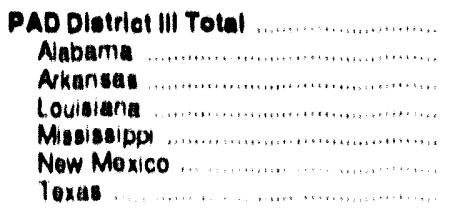 & $\begin{array}{r}718,676 \\
74,740 \\
46,031 \\
71,706 \\
26,621 \\
83,816 \\
446,66 ?\end{array}$ & $\begin{array}{r}807,008 \\
71,312 \\
36,784 \\
46,382 \\
31,545 \\
06,815 \\
511.047\end{array}$ & $\begin{array}{r}827,700 \\
98,924 \\
2,628 \\
428,328 \\
68,037 \\
232,190\end{array}$ & $\begin{array}{r}008,028 \\
94,126 \\
2,292 \\
416,207 \\
70,269 \\
325,043\end{array}$ & $\begin{array}{r}3,390,072 \\
348,437 \\
384,146 \\
366,785 \\
316,681 \\
219,654 \\
1,554,493\end{array}$ & $\begin{array}{r}3,448,003 \\
978,109 \\
390,095 \\
348,020 \\
317,122 \\
225,791 \\
1,516,036\end{array}$ \\
\hline $\begin{array}{l}\text { PAO Distriot IV Total } \\
\text { Colorado } \\
\text { lduho } \\
\text { Montang } \\
\text { Uian } \\
\text { Wyoming }\end{array}$ & $\begin{array}{r}308,082 \\
96,648 \\
26,260 \\
69,478 \\
45,046 \\
62,464\end{array}$ & $\begin{array}{r}208,010 \\
78,011 \\
23,554 \\
63,80 ? \\
47,113 \\
85,824\end{array}$ & $\begin{array}{r}208 \\
206 \\
\end{array}$ & $\begin{array}{r}131 \\
108 \\
23\end{array}$ & $\begin{array}{l}782,786 \\
216,076 \\
124,668 \\
114,876 \\
110,272 \\
156.894\end{array}$ & $\begin{array}{l}820,826 \\
215.220 \\
128.768 \\
132.687 \\
180,007 \\
172.244\end{array}$ \\
\hline 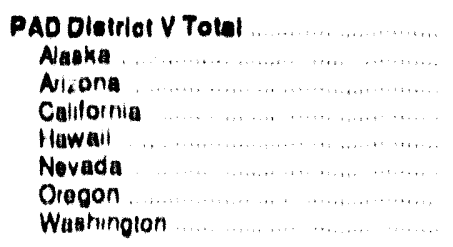 & $\begin{array}{r}402,000 \\
4,703 \\
4,340 \\
237,493 \\
12 \\
6,074 \\
88,347 \\
6 ?, 130\end{array}$ & $\begin{array}{r}346,428 \\
4,500 \\
6,051 \\
184,624 \\
10 \\
3,539 \\
07,441 \\
49,245\end{array}$ & $\begin{array}{r}\$ 16,808 \\
137,829 \\
169,805 \\
66,843 \\
54,202 \\
61,318\end{array}$ & $\begin{array}{r}437,006 \\
130.256 \\
20 \\
95,082 \\
76,226 \\
42.102 \\
03,320\end{array}$ & $\begin{array}{r}2,096,737 \\
39,627 \\
300,734 \\
1,169,575 \\
25,364 \\
140,148 \\
333,566 \\
357,723\end{array}$ & $\begin{array}{r}3,118,870 \\
49,225 \\
351,527 \\
1,642,723 \\
24,850 \\
152,457 \\
329,762 \\
368,334\end{array}$ \\
\hline
\end{tabular}

Son looinotes ar end ol table 
Table 16. Adjueted Sales of Diatlilate Fuel Oll by End Uee, 1991 and 1992 (Continued) (Thousand Gallons)

\begin{tabular}{|c|c|c|c|c|c|c|c|c|}
\hline \multirow{2}{*}{ Deatination } & \multicolumn{2}{|c|}{ Mllitary } & \multicolumn{2}{|c|}{ OH.Hlghway } & \multicolumn{2}{|c|}{ All Other } & \multicolumn{2}{|c|}{ Tolal } \\
\hline & 1901 & 1002 & 1001 & 1002 & 1001 & 1092 & 1001 & 1002 \\
\hline 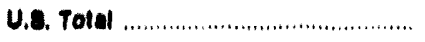 & 828,733 & 680,170 & $1,080,202$ & $1,739,068$ & 2,390 & 7,078 & $44,776,360$ & $48,791,460$ \\
\hline 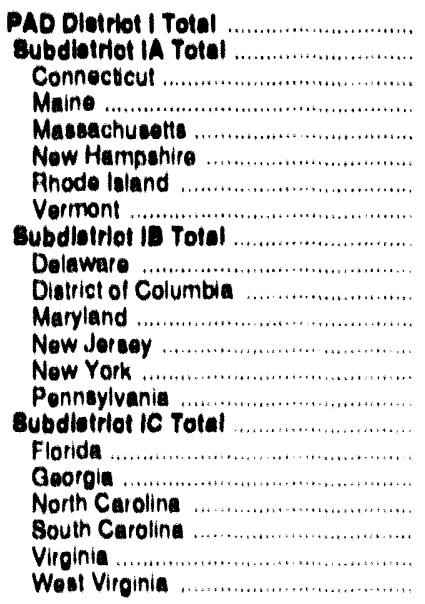 & $\begin{array}{r}249,833 \\
18,831 \\
1,801 \\
3,641 \\
2,633 \\
404 \\
8,729 \\
1,223 \\
39,080 \\
167 \\
4,751 \\
8,407 \\
3,469 \\
20,661 \\
2,225 \\
189,042 \\
7,496 \\
9,685 \\
7,731 \\
97,444 \\
62,732 \\
74\end{array}$ & $\begin{array}{r}122,023 \\
18,274 \\
5,061 \\
2,751 \\
3,172 \\
1,683 \\
2,651 \\
44 \\
39,870 \\
1,688 \\
5,887 \\
21,230 \\
20,809 \\
6,653 \\
3,503 \\
47,770 \\
8,001 \\
8,119 \\
6,679 \\
2,010 \\
21,067 \\
1,003\end{array}$ & $\begin{array}{r}398,783 \\
81,328 \\
14,572 \\
6,983 \\
17,188 \\
7,082 \\
2,055 \\
3,445 \\
198,728 \\
4,035 \\
68 \\
20,031 \\
32,281 \\
37,865 \\
64,447 \\
188,734 \\
47,408 \\
44,417 \\
27,876 \\
20,694 \\
29,626 \\
18,713\end{array}$ & $\begin{array}{r}400,104 \\
48,084 \\
8,660 \\
8,484 \\
15,194 \\
6,667 \\
2,338 \\
5,141 \\
178,134 \\
2,972 \\
458 \\
18,970 \\
23,863 \\
42,210 \\
89,661 \\
173,988 \\
54,663 \\
44,789 \\
25,268 \\
17,132 \\
23,878 \\
8,065\end{array}$ & $\begin{array}{l}- \\
- \\
- \\
- \\
- \\
- \\
- \\
- \\
- \\
- \\
- \\
- \\
- \\
- \\
- \\
-\end{array}$ & 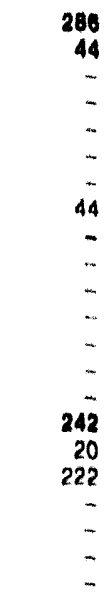 & $\begin{array}{r}16,063,026 \\
3,317,182 \\
633,364 \\
435,172 \\
1,381,328 \\
275,802 \\
212,970 \\
178,546 \\
7,132,470 \\
142,920 \\
99,253 \\
711,246 \\
1,381,626 \\
2,566,694 \\
2,230,730 \\
3,613,374 \\
1,302,827 \\
1,130,399 \\
1,004,179 \\
643,009 \\
1,127,880 \\
405,080\end{array}$ & $\begin{array}{r}16,701,894 \\
3,373,163 \\
934,078 \\
457,627 \\
1,470,275 \\
277,448 \\
223,948 \\
209,787 \\
7,447,710 \\
134,707 \\
80,499 \\
762,314 \\
1,407,342 \\
2,753,653 \\
2,309,195 \\
3,771,022 \\
1,463,171 \\
1,144,146 \\
1,079,932 \\
576,440 \\
1,110,076 \\
397,257\end{array}$ \\
\hline 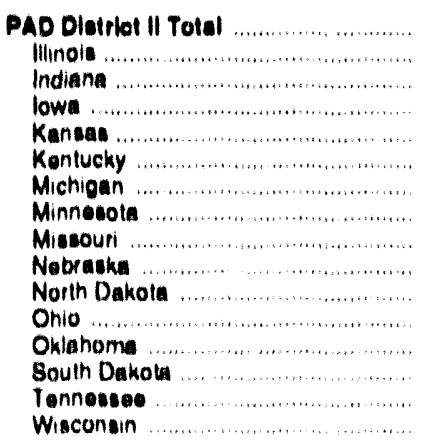 & $\begin{array}{r}43,004 \\
904 \\
5,652 \\
344 \\
2,058 \\
6,811 \\
1,242 \\
1,470 \\
5,252 \\
2,451 \\
1,110 \\
10,531 \\
2,393 \\
559 \\
2,160 \\
878\end{array}$ & $\begin{array}{r}83,702 \\
1,945 \\
7,271 \\
6,923 \\
10,260 \\
10.047 \\
6,897 \\
816 \\
6.228 \\
319 \\
591 \\
9.542 \\
815 \\
5,276 \\
5,844 \\
1.931\end{array}$ & $\begin{array}{r}446,881 \\
58,417 \\
28,071 \\
11,556 \\
14,922 \\
25,007 \\
58,587 \\
40,810 \\
30,942 \\
10,607 \\
5,015 \\
37,114 \\
30,905 \\
5,808 \\
35,043 \\
54,047\end{array}$ & $\begin{array}{r}476,649 \\
52,776 \\
41,989 \\
24,421 \\
17,266 \\
29,535 \\
41,231 \\
35,103 \\
37,057 \\
10,084 \\
2,166 \\
49,831 \\
18,944 \\
8,234 \\
71,313 \\
36,608\end{array}$ & 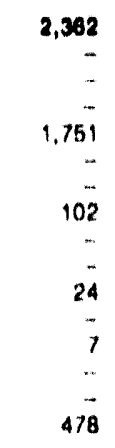 & $\begin{array}{r}807 \\
203 \\
-7 \\
27 \\
10 \\
28 \\
1 \\
\therefore \\
120 \\
- \\
- \\
- \\
418\end{array}$ & $\begin{array}{r}13,021,218 \\
1,518,379 \\
1,361,568 \\
610,722 \\
658,133 \\
954,053 \\
1,056,838 \\
892,256 \\
851,888 \\
546,677 \\
310,588 \\
1,503,002 \\
594,512 \\
245.307 \\
945,550 \\
970,836\end{array}$ & $\begin{array}{r}13,677,676 \\
1,530,052 \\
1,342,920 \\
686,882 \\
635,477 \\
1,079,081 \\
1,076,392 \\
909,732 \\
939,722 \\
591,599 \\
294,892 \\
1,607,986 \\
684,769 \\
234,269 \\
1,009,648 \\
955,255\end{array}$ \\
\hline 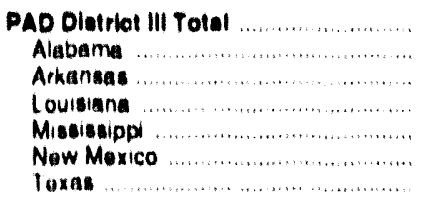 & $\begin{array}{r}243,041 \\
5,228 \\
431 \\
17,763 \\
5,784 \\
1,301 \\
212,534\end{array}$ & $\begin{array}{r}242,345 \\
6,511 \\
577 \\
8,538 \\
19,255 \\
3,695 \\
203,786\end{array}$ & $\begin{array}{r}302,832 \\
54,372 \\
24,825 \\
64,433 \\
25,215 \\
32,743 \\
191,244\end{array}$ & $\begin{array}{r}4,048 \\
71,138 \\
55,932 \\
55,807 \\
35,221 \\
22,164 \\
230,787\end{array}$ & $\begin{array}{l}8 \\
B \\
\cdots \\
- \\
- \\
-\end{array}$ & $\begin{array}{r}5,841 \\
8,035 \\
5,007\end{array}$ & $\begin{array}{r}7,642,320 \\
1,006,018 \\
568,985 \\
1,461,744 \\
649,055 \\
398,668 \\
3,557,650\end{array}$ & $\begin{array}{r}7,687,612 \\
1,028,073 \\
647,247 \\
1,326,479 \\
643,215 \\
417,045 \\
3,795,553\end{array}$ \\
\hline $\begin{array}{l}\text { PAD Diatrlal IV Total } \\
\text { Colorado } \\
\text { ldaho } \\
\text { Montana } \\
\text { Utah } \\
\text { Wyoming }\end{array}$ & $\begin{array}{r}12,280 \\
8,291 \\
1,107 \\
354 \\
2,360 \\
167\end{array}$ & $\begin{array}{r}16,239 \\
6,147 \\
4,841 \\
1,304 \\
1,884 \\
1,863\end{array}$ & $\begin{array}{r}128,631 \\
42,640 \\
21,168 \\
16,963 \\
19,921 \\
24,039\end{array}$ & $\begin{array}{r}114,280 \\
37,005 \\
15,576 \\
21,514 \\
20,738 \\
10,455\end{array}$ & $\frac{18}{11}$ & $\begin{array}{c}93 \\
28 \\
65 \\
- \\
-\end{array}$ & $\begin{array}{r}1,897,390 \\
495,904 \\
357,664 \\
348,697 \\
325,899 \\
369,186\end{array}$ & $\begin{array}{r}1,872,444 \\
521,439 \\
301,966 \\
322,882 \\
337,593 \\
388,563\end{array}$ \\
\hline $\begin{array}{l}\text { PAD Dlotriol V Total } \\
\text { Alaske } \\
\text { Arizona } \\
\text { Calitornia } \\
\text { Hawaii } \\
\text { Novada } \\
\text { Orogon } \\
\text { Washington }\end{array}$ & $\begin{array}{r}268,056 \\
17,282 \\
1,408 \\
188,142 \\
65,153 \\
2,339 \\
703 \\
11,029\end{array}$ & $\begin{array}{r}182,809 \\
19,579 \\
1,469 \\
138,628 \\
8,021 \\
2,640 \\
1,806 \\
9,827\end{array}$ & $\begin{array}{r}318,162 \\
12,936 \\
38,105 \\
150,665 \\
13,782 \\
16,241 \\
34,097 \\
53,337\end{array}$ & $\begin{array}{r}276,077 \\
17,284 \\
22,221 \\
125,056 \\
9,177 \\
18,091 \\
32,527 \\
52,620\end{array}$ & $\begin{array}{c}13 \\
- \\
- \\
- \\
\\
\end{array}$ & 48 & $\begin{array}{r}6,181,446 \\
415,021 \\
432,089 \\
3,173,320 \\
306,803 \\
300,754 \\
680,196 \\
842,663\end{array}$ & $\begin{array}{r}5,691,840 \\
443,354 \\
472,410 \\
2,826,668 \\
222,112 \\
306,746 \\
645,257 \\
775,283\end{array}$ \\
\hline
\end{tabular}

- Estimated data Annual astumato is based on six months ol data. Dashos (c) : No dala toported

Nolo Totals may not equal sum of components due to independent rounding

Sources: - Energy Intormatoon Administration Form ElA-8?!, "Annual Fuol Oil and Kerosene Sales Report," for 1991 and 1992.

- Elociric Ulitity data are based on data obtained on Form tilA 759, "Monthly Power Plant Mepont," and Form FE PrC.423, "Monthly Reporl of Cost and Quality of Fuels lor Elocisic Plants."

- For 1988 grough 1900 , railroad dala for disbllate fugl oll aro based on tho Association of Amorican Pailroads' statistics for railroads of Class I and Amliak Companies. ElA B21 survey reBults are used tor 1991 and 1992.

- On Highway Diesol data are Federal Highway Administation statistics of highway spocial luels uso 
Table 17. Adjusted Sales of Residual Fuel OII by End Use, 1991 and 1992 (Thousand Gallons)

\begin{tabular}{|c|c|c|c|c|c|c|c|c|}
\hline \multirow{2}{*}{ Dostlination } & \multicolumn{2}{|c|}{ Commerolal } & \multicolumn{2}{|c|}{ Industrial } & \multicolumn{2}{|c|}{ Oll Company } & \multicolumn{2}{|c|}{ Eloolrlo Utillity } \\
\hline & 1001 & 1002 & 1001 & 1002 & 1091 & 1002 & 1901 & 1002 \\
\hline 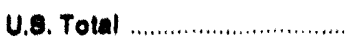 & $1,426,888$ & $1,289,987$ & $1,938,473$ & $2,332,911$ & 301,811 & 297,332 & $7,189,899$ & $8,887,288$ \\
\hline 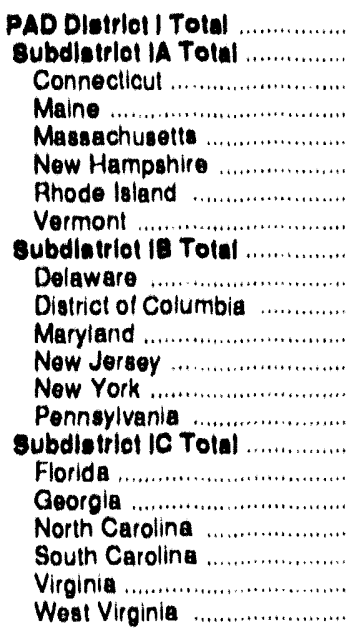 & $\begin{array}{r}1,311,670 \\
378,699 \\
22,267 \\
103,667 \\
191,057 \\
28,416 \\
24,761 \\
5,631 \\
830,888 \\
2,154 \\
9,340 \\
5,617 \\
67,608 \\
718,867 \\
26,610 \\
104,184 \\
90,310 \\
910 \\
4,052 \\
1,038 \\
4,830 \\
2,144\end{array}$ & $\begin{array}{r}1,192,812 \\
289,121 \\
37,883 \\
63,311 \\
167,396 \\
13,826 \\
22,203 \\
4,804 \\
807,739 \\
3,789 \\
11,411 \\
20,279 \\
58,166 \\
676,848 \\
37,548 \\
98,681 \\
76,511 \\
268 \\
4,763 \\
2,238 \\
9,614 \\
2,369\end{array}$ & $\begin{array}{r}1,369,198 \\
368,972 \\
41,667 \\
224,282 \\
59,170 \\
19,408 \\
15,927 \\
5,517 \\
385,773 \\
26,678 \\
54 \\
30,386 \\
128,837 \\
98,329 \\
101,489 \\
617,460 \\
109,830 \\
73,481 \\
220,654 \\
67,412 \\
103,190 \\
42,883\end{array}$ & $\begin{array}{r}1,716,820 \\
470,133 \\
51,874 \\
255,377 \\
92,482 \\
43,747 \\
19,498 \\
7,185 \\
418,461 \\
38,063 \\
83 \\
43,461 \\
129,509 \\
127,230 \\
83,106 \\
828,236 \\
174,821 \\
144,881 \\
286,587 \\
75,852 \\
123,605 \\
22,280\end{array}$ & $\begin{array}{r}108,087 \\
228 \\
228 \\
- \\
- \\
- \\
- \\
- \\
107,228 \\
13,111 \\
2,304 \\
3,418 \\
1,941 \\
86,451 \\
1,547 \\
96 \\
42 \\
160 \\
1,248 \\
-\end{array}$ & $\begin{array}{r}123,428 \\
228 \\
228 \\
- \\
- \\
- \\
- \\
- \\
121,206 \\
17,465 \\
- \\
2,052 \\
2,557 \\
4,030 \\
96,171 \\
1,933 \\
126 \\
288 \\
-\overline{1} \\
194 \\
1,326 \\
-\end{array}$ & 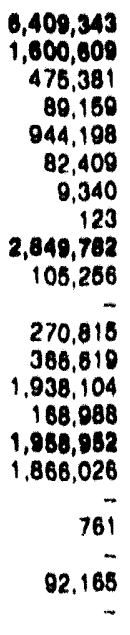 & $\begin{array}{r}8,029,803 \\
1,383,033 \\
383,144 \\
04,013 \\
809,648 \\
101,196 \\
5,886 \\
70 \\
1,746,190 \\
00,148 \\
164,268 \\
99,669 \\
1,267,802 \\
136,303 \\
1,020,080 \\
1,848,660 \\
- \\
- \\
081 \\
70,069 \\
-\end{array}$ \\
\hline 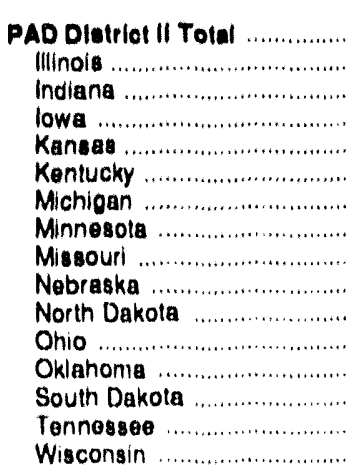 & $\begin{array}{r}40,818 \\
1,681 \\
8,615 \\
377 \\
292 \\
- \\
201 \\
12,401 \\
1,248 \\
1,119 \\
317 \\
1,695 \\
3,209 \\
1,473 \\
697 \\
7,519\end{array}$ & $\begin{array}{r}38,102 \\
1,842 \\
762 \\
1,580 \\
929 \\
- \\
503 \\
8,369 \\
132 \\
1,739 \\
513 \\
3.136 \\
1,836 \\
1.511 \\
2.437 \\
9,812\end{array}$ & $\begin{array}{r}286,183 \\
10,475 \\
70,182 \\
3,677 \\
2,679 \\
10,137 \\
26,551 \\
20,748 \\
20,034 \\
7,166 \\
4,056 \\
44,655 \\
6,473 \\
1,357 \\
14,156 \\
23,838\end{array}$ & $\begin{array}{r}347,094 \\
8,451 \\
105,343 \\
2,853 \\
5,108 \\
8,964 \\
31,167 \\
28,379 \\
25,962 \\
6,187 \\
4,325 \\
58,863 \\
24,365 \\
4,615 \\
12,247 \\
21,116\end{array}$ & $\begin{array}{r}121,040 \\
25,346 \\
53,701 \\
2,286 \\
9,131 \\
4,923 \\
10,713 \\
- \\
5 \\
8,502 \\
2,803 \\
- \\
111 \\
4,431\end{array}$ & $\begin{array}{r}107,034 \\
7,358 \\
59,490 \\
1,550 \\
8,919 \\
1,124 \\
12,551 \\
392 \\
-40 \\
7,492 \\
3,754 \\
\\
- \\
264 \\
4,739\end{array}$ & $\begin{array}{r}139,163 \\
82,485 \\
- \\
- \\
36,840 \\
477 \\
12,078 \\
310 \\
7.993 \\
- \\
- \\
-\end{array}$ & $\begin{array}{r}128,420 \\
88,838 \\
- \\
26,853 \\
- \\
14,746 \\
39 \overline{-} \\
194 \\
- \\
-\end{array}$ \\
\hline 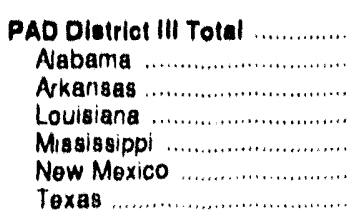 & $\begin{array}{r}24,803 \\
10,251 \\
5,088 \\
25 \\
9,140\end{array}$ & $\begin{array}{r}1,117 \\
171 \\
257 \\
19 \\
670\end{array}$ & $\begin{array}{r}117,560 \\
3,580 \\
6,101 \\
47,324 \\
10,020 \\
4,925 \\
45,599\end{array}$ & $\begin{array}{r}105,614 \\
15,739 \\
1,139 \\
42,535 \\
8,133 \\
5,423 \\
32,544\end{array}$ & $\begin{array}{r}708 \\
- \\
- \\
- \\
\end{array}$ & $\begin{array}{r}2,324 \\
\ldots \\
\cdots \\
\cdots \\
2,324\end{array}$ & $\begin{array}{r}27,294 \\
49 \\
4,606 \\
26,638 \\
-\end{array}$ & $\begin{array}{r}23,886 \\
- \\
696 \\
22,859 \\
-\end{array}$ \\
\hline 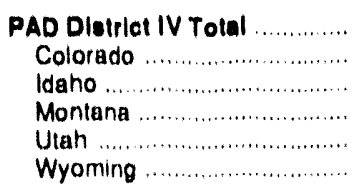 & $\begin{array}{r}1,200 \\
- \\
52 \\
138 \\
965 \\
45\end{array}$ & $\begin{array}{r}1,640 \\
10 \\
588 \\
166 \\
876 \\
2\end{array}$ & $\begin{array}{r}6,617 \\
1,419 \\
1,813 \\
228 \\
3,099 \\
58\end{array}$ & $\begin{array}{r}7,190 \\
159 \\
337 \\
3,627 \\
2,776 \\
281\end{array}$ & $\begin{array}{r}11,783 \\
- \\
5,773 \\
4,412 \\
1,598\end{array}$ & $\begin{array}{r}6,980 \\
\cdots \\
\cdots \\
6,866 \\
90\end{array}$ & $\begin{array}{l}- \\
- \\
- \\
-\end{array}$ & $\begin{array}{l}- \\
- \\
-\end{array}$ \\
\hline 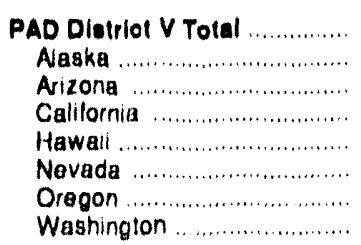 & $\begin{array}{r}48,468 \\
-\overline{4} \\
463 \\
32,135 \\
782 \\
97 \\
10,754 \\
4,237\end{array}$ & $\begin{array}{r}89,617 \\
1,812 \\
45,107 \\
17 \\
10,294 \\
2,387\end{array}$ & $\begin{array}{r}178,928 \\
11,774 \\
7,421 \\
54,038 \\
35,763 \\
3,438 \\
14,593 \\
51,900\end{array}$ & $\begin{array}{r}186,293 \\
12,800 \\
3,990 \\
58,961 \\
17,828 \\
3,392 \\
21,352 \\
37,969\end{array}$ & $\begin{array}{r}38,074 \\
- \\
20,079 \\
33,519 \\
- \\
4,476\end{array}$ & $\begin{array}{r}86,992 \\
21,069 \\
31,632 \\
- \\
4,291\end{array}$ & $\begin{array}{r}693,809 \\
102,077 \\
481,440 \\
10,282 \\
- \\
-\end{array}$ & $\begin{array}{r}404,482 \\
- \\
72 \\
387,243 \\
17,137 \\
-\end{array}$ \\
\hline
\end{tabular}

Seo footnotes at end of table. 
Table 17. Adjusted Sales of Residual Fuel Oll by End Use, 1991 and 1992 (Continued) (Thousand Gallons)

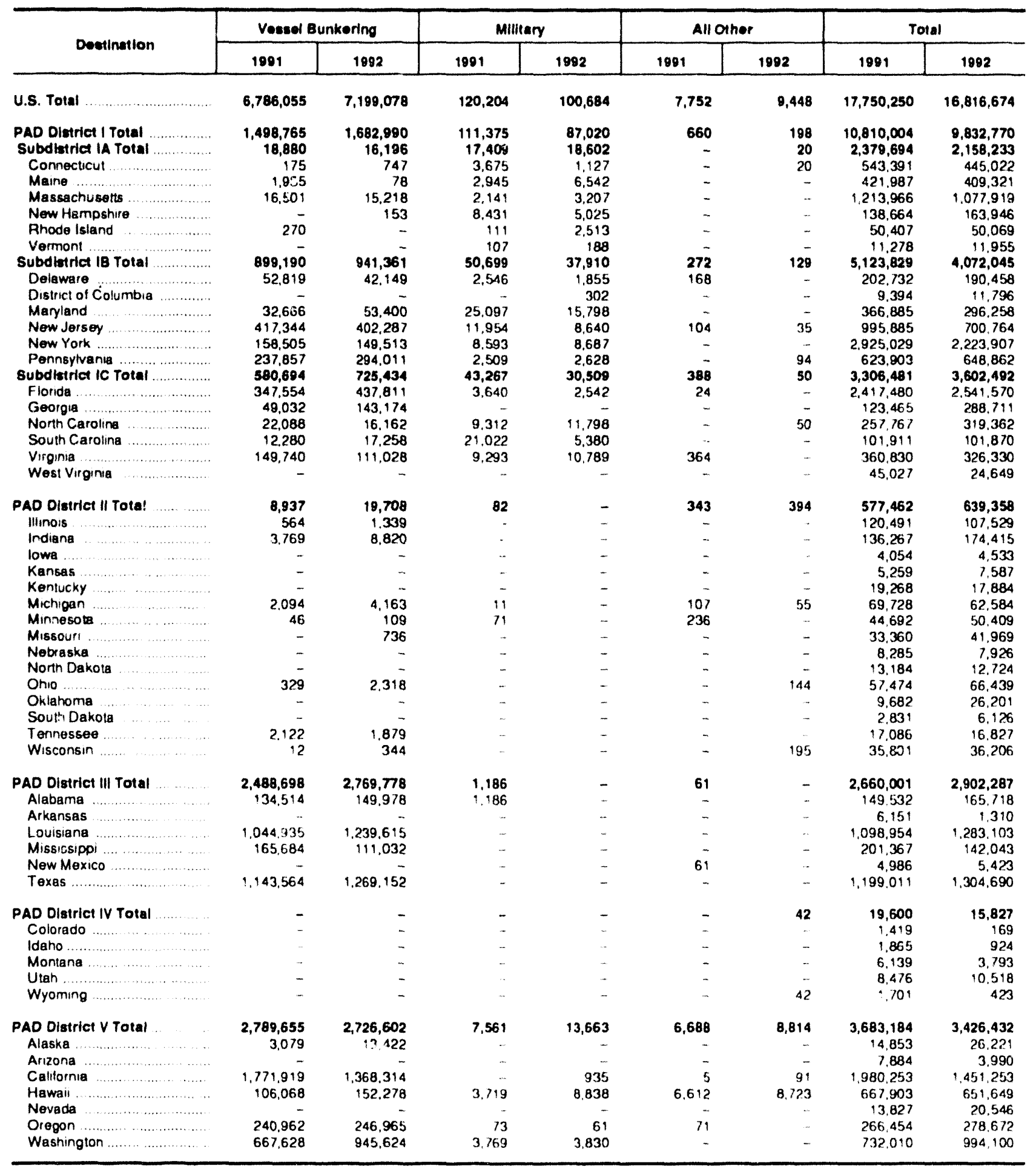

Dashes $(i)=$ No data reported

Note: Totais may not equal sum of components due to independent roundirg

Sources: - Energy Information Administration Form EIA.821. "Annual Fuel Oil and Kerosene Sales Repont." for 1991 and 1992

- Electric Utility data are based on data oblained on Form ElA.759. "Monthly Power Piant Report," and Form FERC-423, "Monthiy Report of Cost and Quality of Fuels for Electric Plants.

- For 1988 through 1990, military dab for residual tuel oll are based on the Delense Fuel Supply Center's "DEIS Report." EIA-821 survey results are used for 1991 and 1992

- Energy information Administration's "Petroleuni Supply Annual," Volume 1, 1991 and 1992, (DOE/E/A.0340(91)/1- (92)/1) 
Table 18. Adjusted Sales of Kerosene by End Use, 1991 and 1992

\section{(Thousand Gallons)}

\begin{tabular}{|c|c|c|c|c|c|c|}
\hline \multirow{2}{*}{ Destination } & \multicolumn{2}{|c|}{ Rosldential } & \multicolumn{2}{|c|}{ Commercial } & \multicolumn{2}{|c|}{ Industrlal } \\
\hline & 1991 & 1992 & 1991 & 1992 & 1901 & 1092 \\
\hline 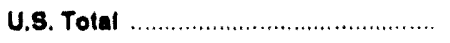 & 491,929 & 481,511 & 97,186 & 82,398 & 76,065 & 46,098 \\
\hline 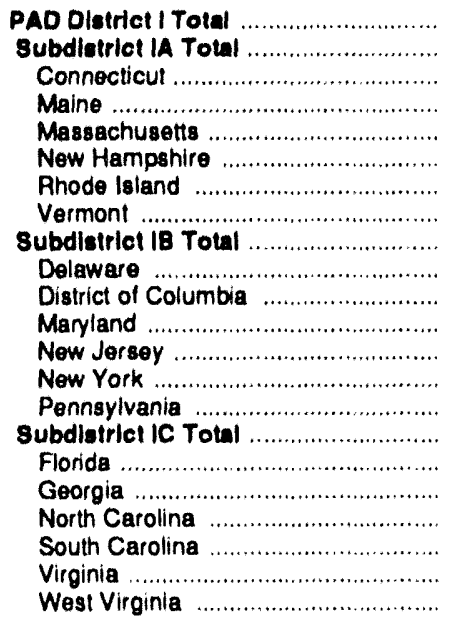 & $\begin{array}{r}283,716 \\
40,891 \\
4,855 \\
16,482 \\
4,192 \\
7,490 \\
985 \\
6,867 \\
125,148 \\
4,602 \\
111 \\
11,014 \\
9,144 \\
58,352 \\
41,825 \\
117,676 \\
5,427 \\
3,148 \\
46,539 \\
20,316 \\
36,764 \\
5,481\end{array}$ & $\begin{array}{r}385,784 \\
\mathbf{8 9 , 8 4 2} \\
8,211 \\
18,887 \\
10,887 \\
10,502 \\
1,533 \\
8,812 \\
150,089 \\
6,039 \\
148 \\
13,284 \\
11,459 \\
52,603 \\
66,555 \\
175,863 \\
11,500 \\
4,574 \\
77,044 \\
18,528 \\
53,906 \\
10,300\end{array}$ & $\begin{array}{r}42,642 \\
14,741 \\
4,648 \\
3,467 \\
5,568 \\
595 \\
32 \\
431 \\
16,605 \\
359 \\
111 \\
1,451 \\
5,330 \\
5,924 \\
3,630 \\
11,096 \\
804 \\
1,470 \\
2,596 \\
323 \\
4,114 \\
1,789\end{array}$ & $\begin{array}{r}61,068 \\
9,367 \\
1,894 \\
2,757 \\
3,054 \\
926 \\
130 \\
596 \\
39,773 \\
50 \\
181 \\
1,767 \\
16,348 \\
17,148 \\
4,279 \\
11,939 \\
1,246 \\
1,535 \\
1,931 \\
572 \\
5,325 \\
1,329\end{array}$ & $\begin{array}{r}21,136 \\
1,686 \\
484 \\
203 \\
289 \\
166 \\
420 \\
94 \\
11,510 \\
120 \\
- \\
427 \\
2,152 \\
5,862 \\
2,949 \\
7,970 \\
263 \\
559 \\
2,860 \\
2,299 \\
1,386 \\
603\end{array}$ & $\begin{array}{r}28,430 \\
6,404 \\
279 \\
256 \\
3,764 \\
549 \\
464 \\
92 \\
13,623 \\
80 \\
- \\
365 \\
6,057 \\
3,834 \\
3,287 \\
9,402 \\
172 \\
301 \\
3,688 \\
2,508 \\
1,176 \\
1,557\end{array}$ \\
\hline 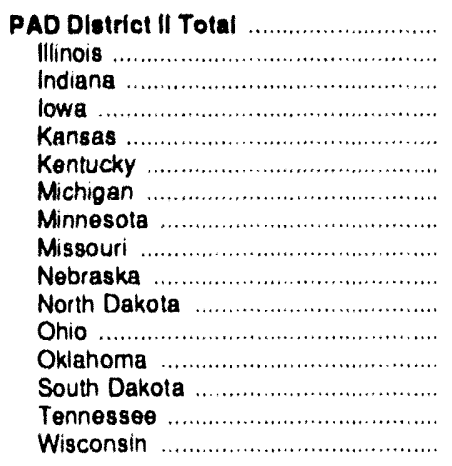 & $\begin{array}{r}153,446 \\
8,090 \\
21,903 \\
2,335 \\
691 \\
26,199 \\
19,350 \\
2,818 \\
2,573 \\
379 \\
515 \\
46,817 \\
691 \\
311 \\
18,576 \\
2,098\end{array}$ & $\begin{array}{r}86,392 \\
2,544 \\
7,793 \\
847 \\
553 \\
15,337 \\
8,595 \\
1,605 \\
868 \\
427 \\
258 \\
30,580 \\
449 \\
155 \\
15,158 \\
1,222\end{array}$ & $\begin{array}{r}31,176 \\
2,752 \\
2,989 \\
178 \\
253 \\
7,071 \\
1,189 \\
239 \\
306 \\
186 \\
35 \\
12,504 \\
667 \\
8 \\
2,188 \\
610\end{array}$ & $\begin{array}{r}14,258 \\
1,444 \\
2,460 \\
166 \\
188 \\
2,433 \\
213 \\
282 \\
679 \\
62 \\
14 \\
2,837 \\
154 \\
16 \\
2,884 \\
427\end{array}$ & $\begin{array}{r}19,154 \\
2,628 \\
2,762 \\
99 \\
235 \\
3,441 \\
2,608 \\
44 \\
260 \\
87 \\
3 \\
4,386 \\
425 \\
- \\
1,946 \\
230\end{array}$ & $\begin{array}{r}11,149 \\
1,389 \\
1,054 \\
51 \\
44 \\
2,622 \\
1,216 \\
14 \\
68 \\
32 \\
4 \\
3,839 \\
292 \\
11 \\
325 \\
188\end{array}$ \\
\hline 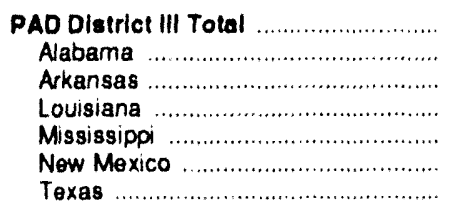 & $\begin{array}{r}26,820 \\
10,769 \\
2,484 \\
2,404 \\
3,998 \\
1,085 \\
6,080\end{array}$ & $\begin{array}{r}3,724 \\
1,275 \\
308 \\
394 \\
600 \\
191 \\
956\end{array}$ & $\begin{array}{r}13,684 \\
2,644 \\
328 \\
3,877 \\
1,087 \\
3,545 \\
2,204\end{array}$ & $\begin{array}{r}4,978 \\
733 \\
214 \\
430 \\
368 \\
368 \\
2,866\end{array}$ & $\begin{array}{r}25,344 \\
3,145 \\
1,725 \\
8,015 \\
898 \\
6,498 \\
5,062\end{array}$ & $\begin{array}{r}4,729 \\
1,376 \\
84 \\
830 \\
436 \\
357 \\
1,646\end{array}$ \\
\hline 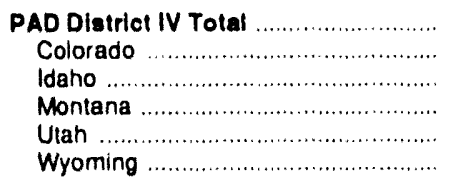 & $\begin{array}{r}2,823 \\
1,971 \\
129 \\
67 \\
412 \\
245\end{array}$ & $\begin{array}{r}1,775 \\
1,550 \\
68 \\
8 \\
95 \\
53\end{array}$ & $\begin{array}{r}1,841 \\
869 \\
16 \\
20 \\
695 \\
240\end{array}$ & $\begin{array}{r}346 \\
282 \\
7 \\
8 \\
39 \\
10\end{array}$ & $\begin{array}{r}632 \\
82 \\
110 \\
2 \\
186 \\
251\end{array}$ & $\begin{array}{r}318 \\
52 \\
11 \\
1 \\
27 \\
227\end{array}$ \\
\hline 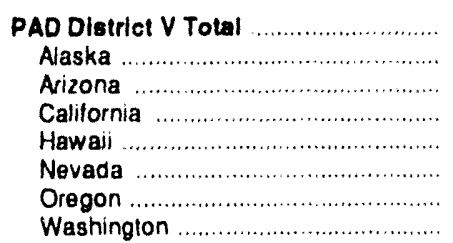 & $\begin{array}{r}25,125 \\
1,247 \\
110 \\
12,676 \\
1 \\
1,632 \\
2.138 \\
7,322\end{array}$ & $\begin{array}{r}3,836 \\
28 \\
82 \\
1,367 \\
- \\
403 \\
716 \\
1,239\end{array}$ & $\begin{array}{r}7,843 \\
19 \\
296 \\
3,671 \\
24 \\
484 \\
605 \\
2.744\end{array}$ & $\begin{array}{r}1,748 \\
10 \\
27 \\
835 \\
3 \\
172 \\
196 \\
503\end{array}$ & $\begin{array}{r}8,789 \\
26 \\
5,117 \\
2.112 \\
15 \\
1.190 \\
383 \\
946\end{array}$ & $\begin{array}{r}1,475 \\
11 \\
14 \\
588 \\
9 \\
389 \\
309 \\
156\end{array}$ \\
\hline
\end{tabular}

See foolnotes at end of table. 
Table 18. Adjusted Sales of Kerosene by End Use, 1991 and 1992 (Continued) (Thousand Gallons)

\begin{tabular}{|c|c|c|c|c|c|c|}
\hline \multirow{2}{*}{ Destination } & \multicolumn{2}{|c|}{ Farm } & \multicolumn{2}{|c|}{ All Other } & \multicolumn{2}{|c|}{ Total } \\
\hline & 1001 & 1992 & 1891 & 1992 & 1891 & 1002 \\
\hline 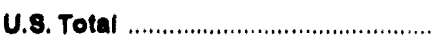 & 24,808 & 20,036 & 19,678 & 6,382 & 709,716 & 636,426 \\
\hline 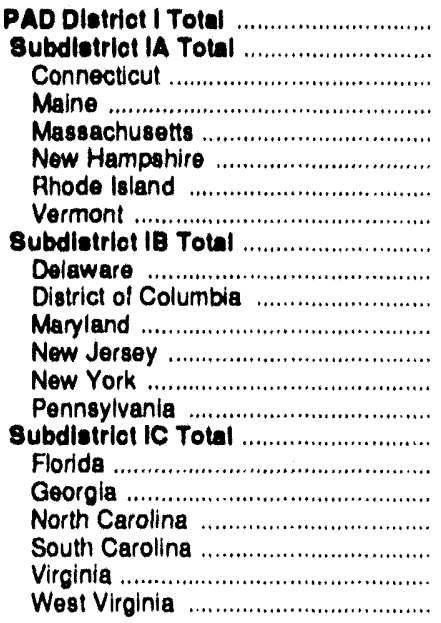 & $\begin{array}{r}5,867 \\
638 \\
1 \\
432 \\
21 \\
18 \\
-\overline{1} \\
167 \\
2,462 \\
50 \\
-\overline{2} \\
267 \\
1,702 \\
431 \\
2,776 \\
47 \\
65 \\
1,376 \\
512 \\
499 \\
277\end{array}$ & $\begin{array}{r}10,948 \\
488 \\
51 \\
233 \\
28 \\
48 \\
1 \\
127 \\
6,165 \\
37 \\
34 \\
347 \\
28 \\
3,782 \\
1,972 \\
4,293 \\
35 \\
43 \\
2,359 \\
150 \\
916 \\
791\end{array}$ & $\begin{array}{r}6,328 \\
1,567 \\
550 \\
76 \\
201 \\
689 \\
4 \\
48 \\
2,973 \\
58 \\
- \\
84 \\
484 \\
1,749 \\
588 \\
1,787 \\
50 \\
159 \\
485 \\
221 \\
671 \\
202\end{array}$ & $\begin{array}{r}3,687 \\
476 \\
11 \\
94 \\
63 \\
263 \\
2 \\
44 \\
2,210 \\
11 \\
- \\
101 \\
569 \\
836 \\
692 \\
1,001 \\
177 \\
62 \\
89 \\
250 \\
255 \\
168\end{array}$ & $\begin{array}{r}359,888 \\
\mathbf{5 9 , 4 9 8} \\
10,538 \\
20,660 \\
10,271 \\
8,958 \\
1,441 \\
7,627 \\
158,888 \\
5,190 \\
222 \\
13,243 \\
17,112 \\
73,588 \\
49,533 \\
141,308 \\
6,591 \\
5,401 \\
53,857 \\
23,672 \\
43,434 \\
8,351\end{array}$ & $\begin{array}{r}480,013 \\
76,807 \\
10,445 \\
23,227 \\
17,807 \\
12,288 \\
2,130 \\
9,670 \\
211,859 \\
6,217 \\
328 \\
15,864 \\
34,461 \\
78,204 \\
76,785 \\
202,488 \\
13,131 \\
6,514 \\
85,111 \\
22,009 \\
61,579 \\
14,145\end{array}$ \\
\hline 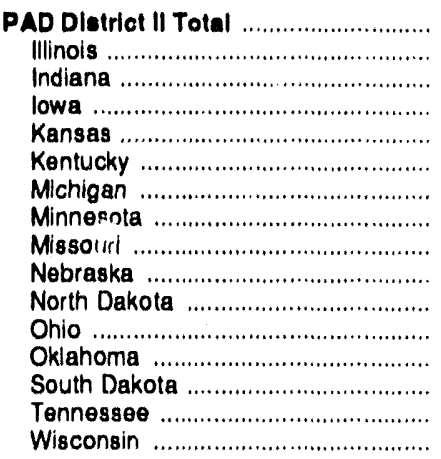 & $\begin{array}{r}8,944 \\
417 \\
378 \\
749 \\
473 \\
803 \\
756 \\
635 \\
188 \\
534 \\
114 \\
3,023 \\
160 \\
180 \\
299 \\
233\end{array}$ & $\begin{array}{r}6,737 \\
298 \\
1,060 \\
669 \\
445 \\
575 \\
461 \\
297 \\
163 \\
309 \\
14 \\
1,715 \\
308 \\
144 \\
81 \\
200\end{array}$ & $\begin{array}{r}5,219 \\
193 \\
145 \\
166 \\
36 \\
730 \\
1,052 \\
26 \\
1,157 \\
22 \\
18 \\
517 \\
226 \\
8 \\
708 \\
215\end{array}$ & $\begin{array}{r}1,437 \\
273 \\
136 \\
27 \\
148 \\
252 \\
41 \\
27 \\
37 \\
2 \\
1 \\
173 \\
103 \\
- \\
114 \\
105\end{array}$ & $\begin{array}{r}217,938 \\
14,080 \\
28,177 \\
3,527 \\
1,688 \\
38,245 \\
24,955 \\
3,762 \\
4,483 \\
1,208 \\
686 \\
67,347 \\
2,169 \\
508 \\
23,717 \\
3,387\end{array}$ & $\begin{array}{r}119,972 \\
5,947 \\
12,502 \\
1,759 \\
1,378 \\
21,218 \\
10,525 \\
2,225 \\
1,814 \\
833 \\
291 \\
39,144 \\
1,305 \\
327 \\
18,563 \\
2,142\end{array}$ \\
\hline 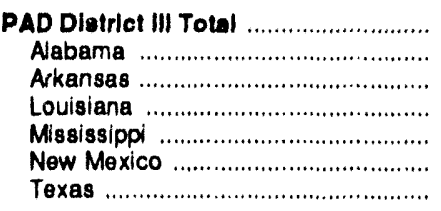 & $\begin{array}{r}6,091 \\
35 \\
888 \\
431 \\
3,320 \\
201 \\
1,216\end{array}$ & $\begin{array}{r}1,704 \\
41 \\
190 \\
205 \\
37 \\
15 \\
1,216\end{array}$ & $\begin{array}{r}\mathbf{5 , 8 8 7} \\
502 \\
934 \\
760 \\
1,562 \\
151 \\
1,977\end{array}$ & $\begin{array}{r}1,055 \\
68 \\
112 \\
85 \\
168 \\
30 \\
593\end{array}$ & $\begin{array}{r}77,826 \\
17,095 \\
6,359 \\
15,487 \\
10,865 \\
11,481 \\
16,539\end{array}$ & $\begin{array}{r}16,190 \\
3,493 \\
907 \\
1,943 \\
1,609 \\
961 \\
7,278\end{array}$ \\
\hline 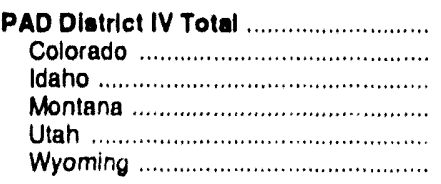 & $\begin{array}{r}1,139 \\
903 \\
37 \\
149 \\
21 \\
30\end{array}$ & $\begin{array}{r}265 \\
234 \\
11 \\
1 \\
13 \\
7\end{array}$ & $\begin{array}{r}483 \\
368 \\
- \\
8 \\
71 \\
7\end{array}$ & $\begin{array}{r}42 \\
29 \\
4 \\
6 \\
1 \\
2\end{array}$ & $\begin{array}{r}6,888 \\
4,193 \\
292 \\
245 \\
1,384 \\
773\end{array}$ & $\begin{array}{r}2,746 \\
2,147 \\
102 \\
24 \\
175 \\
299\end{array}$ \\
\hline 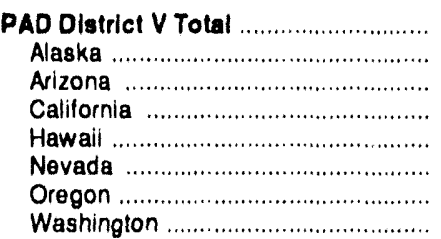 & $\begin{array}{r}2,827 \\
-\overline{7} \\
2,528 \\
\overline{77} \\
132 \\
60\end{array}$ & $\begin{array}{r}384 \\
\overline{6} \\
233 \\
\overline{7} \\
51 \\
87\end{array}$ & $\begin{array}{r}1,791 \\
1 \\
221 \\
1,158 \\
- \\
213 \\
116 \\
82\end{array}$ & $\begin{array}{r}161 \\
3 \\
1 \\
128 \\
\overline{5} \\
23 \\
2\end{array}$ & $\begin{array}{r}47,376 \\
1,294 \\
5,774 \\
22,144 \\
40 \\
3,597 \\
3,374 \\
11,154\end{array}$ & $\begin{array}{r}7,604 \\
52 \\
130 \\
3,152 \\
13 \\
976 \\
1,285 \\
1,987\end{array}$ \\
\hline
\end{tabular}

Dashes $(\cdot)$ = No data reported.

Note: Totals may not equal sum of components due to independent rounding.

Sources: - Energy Information Administration Form ElA.821, "Annual Fuel Oll and Kerosene Sales Report," lor 1991 and 1992.

- Energy intormation Administration's "Petroleum Supply Annual," Volume 1,1991 and 1992, (DOE/E/A-0340(91)/1 - (92)/1). 
Table 19. Adjusted Sales for Residential Use: Distillate Fuel Oll and Kerosene, 1992 (Thousand Gallons)

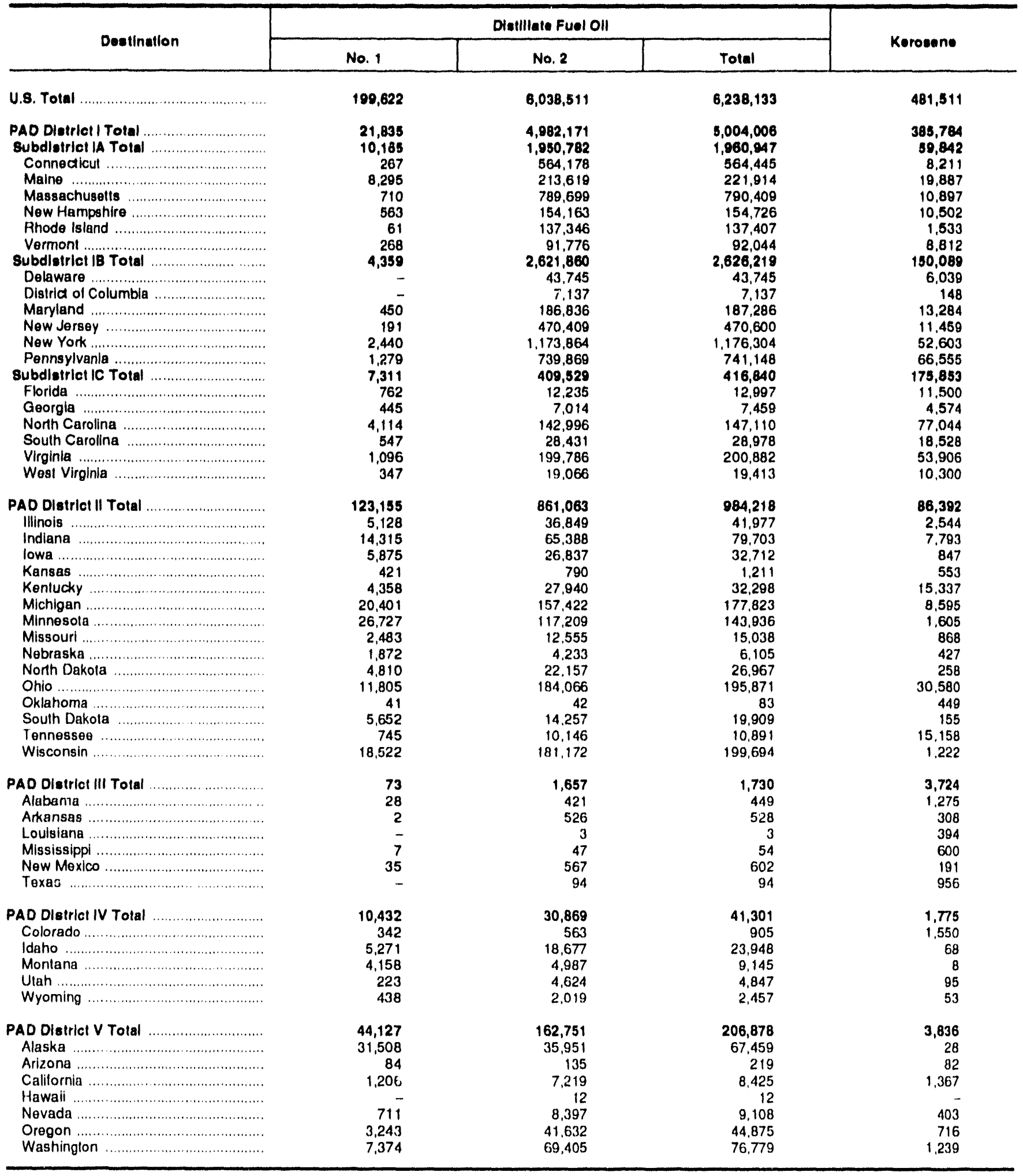

Dashes $(-)=$ No dala reported

Note: Totals may not equal sum of components due to independent rounding.

Sources: - Energy Inlormation Administration Form ElA-821. "Annual Fuel Oil and Kerosene Sales Peport," lor 1992

- Energy Informallon Administration's "Petroloum Supply Annual," Volume 1, 1992, (DOE/EIA-0340(92)/1). 
Table 20. Adjusted Sales for Commercial Use: Distillate Fuel Oll, Residual Fuel Oll, and Kerosene, 1992

(Thousand Gallons)

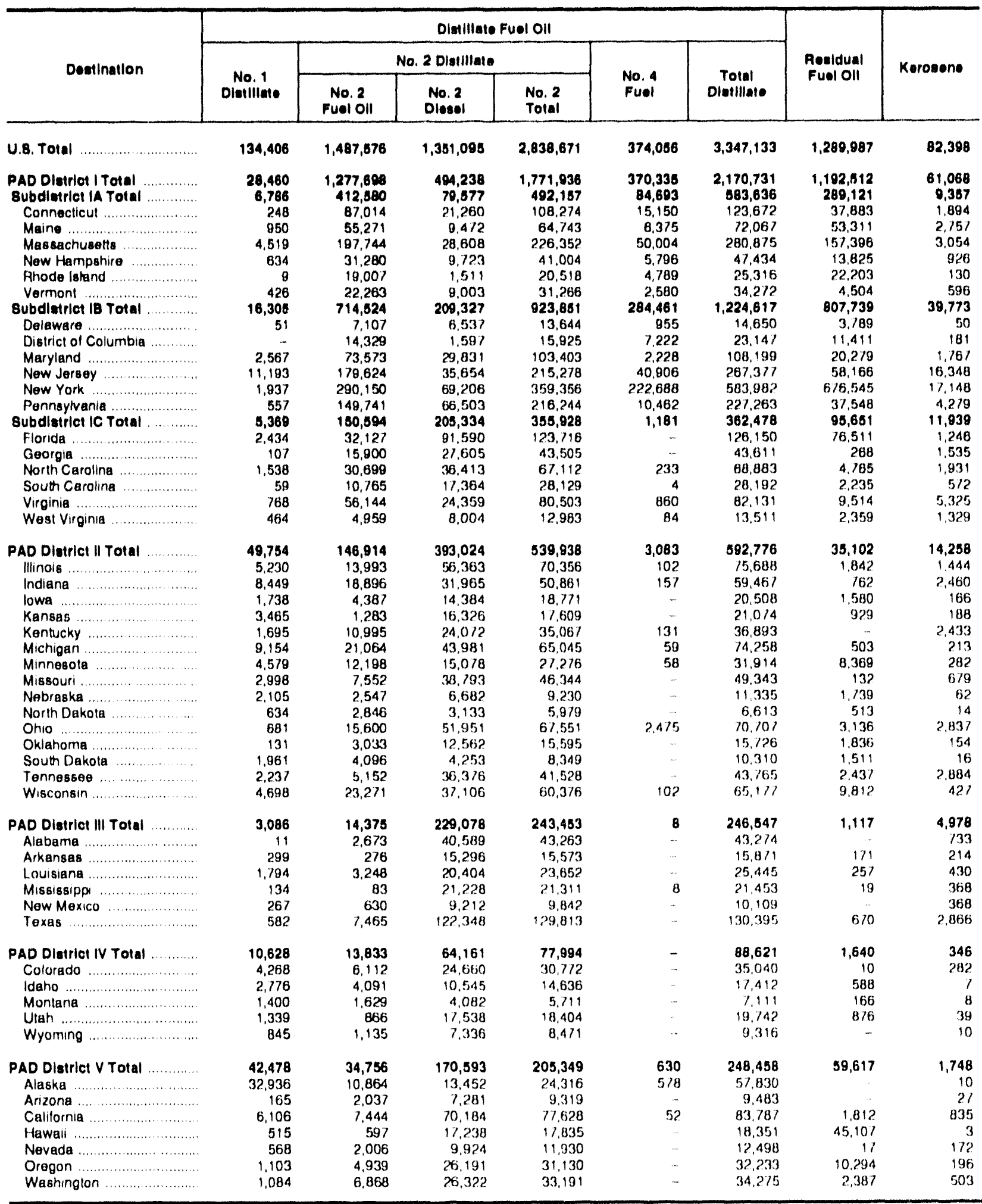

Dashes (-) = No data reported.

Note. Totals may not equal sum of components due to independent rounding

Sources: - Energy Intormation Administration form EIA.821. "Annual Fuel Oil and Kerosene Sales Report," for 1992

- Energy Intormation Administration's "Petroleum Supply Annual," Volume 1, 1992, (DOE/EIA 0340(92)/1) 
Table 21. Adjusted Sales for Industrial and OII Company Use: Distillate Fuel Oil, Residual Fuel OII, and Kerosene, 1992

(Thousand Gallons)

\begin{tabular}{|c|c|c|c|c|c|c|c|c|c|c|}
\hline \multirow{3}{*}{ Destination } & \multicolumn{8}{|c|}{ Indusirial Use } & \multicolumn{2}{|c|}{ Oll Company Use } \\
\hline & \multirow[b]{2}{*}{$\begin{array}{c}\text { No. } 1 \\
\text { Distillate }\end{array}$} & \multicolumn{3}{|c|}{ No. 2 Distliliate } & \multirow{2}{*}{$\begin{array}{l}\text { No. } 4 \\
\text { Fuel }\end{array}$} & \multirow[b]{2}{*}{$\begin{array}{c}\text { Total } \\
\text { Diatillate }\end{array}$} & \multirow{2}{*}{$\begin{array}{l}\text { Residual } \\
\text { Fuel Oll }\end{array}$} & \multirow[b]{2}{*}{ Kerosene } & \multirow[b]{2}{*}{$\begin{array}{l}\text { Distillate } \\
\text { Fuel oil }\end{array}$} & \multirow[b]{2}{*}{$\begin{array}{l}\text { Resldual } \\
\text { Fuel Oll }\end{array}$} \\
\hline & & $\begin{array}{l}\text { No. } 2 \\
\text { Fuel Oll }\end{array}$ & $\begin{array}{l}\text { No. } 2 \\
\text { Dlesel }\end{array}$ & $\begin{array}{l}\text { No. } 2 \\
\text { Tolal }\end{array}$ & & & & & & \\
\hline U.8. Total & 63,218 & 822,400 & $1,860,057$ & $2,082,507$ & 73,118 & $2,218,843$ & $2,332,811$ & 46,099 & 785,120 & 297,332 \\
\hline $\begin{array}{l}\text { PAD Diatrlet I Total } \\
\text { Subdiatrict IA Total }\end{array}$ & $\begin{array}{r}3,939 \\
562\end{array}$ & $\begin{array}{r}278,815 \\
93,114\end{array}$ & $\begin{array}{r}307,360 \\
14,725\end{array}$ & $\begin{array}{l}386,175 \\
107,839\end{array}$ & $\begin{array}{l}61,005 \\
21,959\end{array}$ & $\begin{array}{l}681,118 \\
130,360\end{array}$ & $\begin{array}{r}1,716,820 \\
470,133\end{array}$ & $\begin{array}{r}28,430 \\
5,404\end{array}$ & $\begin{array}{r}13,076 \\
1,097\end{array}$ & $\begin{array}{r}123,426 \\
228\end{array}$ \\
\hline Connecticut & 22 & 17,078 & 1,747 & $18,8 ? 5$ & 5,683 & 24,530 & 51,814 & 310 & 336 & 228 \\
\hline Maine & 43 & $15,78 ?$ & $1,74 B$ & 11,530 & 1.994 & 19.567 & $255.3 / 7$ & 256 & 96 & $-\cdots$ \\
\hline Massachuselts & 480 & 40,754 & 8,559 & 49.313 & 8.620 & 58.421 & 92,452 & 3.764 & 344 & \\
\hline New Hampshure & 8 & 9.055 & $5 / 3$ & 9.629 & 2.1565 & 11.198 & 43,141 & 540 & 50 & \\
\hline Phode Island & & 6,617 & 488 & 1,165 & 1.554 & B. 119 & 19.4918 & 464 & 242 & \\
\hline Vermont ..... & 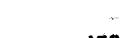 & 3.768 & 1,610 & 5,379 & 1,952 & 1,331 & 7.185 & 92 & 19 & \\
\hline Subdistrlet IB Tolal & 479 & 93,310 & 113,116 & 206,426 & 28,927 & 236,832 & 418,451 & 13,623 & 7,984 & 121,265 \\
\hline Dolaware & 1 & 1,610 & 3,573 & 5,183 & 1.098 & 6.281 & 35,063 & 80 & $286 i$ & $1 / 455$ \\
\hline District ol Columbia & - & 12 & 57 & 603 & 11 & 19 & 83 & & & \\
\hline Maryland ....... & 311 & 7.403 & 11,635 & $19,0,34$ & 1.167 & 20,516 & 43,461 & 365 & 651 & $2.05 ?$ \\
\hline Now Jersoy & 16 & 30,843 & 6.823 & 37,765 & 11,998 & 49,781 & 120,509 & 6.051 & $2.5 / 1$ & 2.557 \\
\hline Now York & 25 & 19,349 & 11.201 & 30,553 & 9.416 & 39.994 & 127,230 & 3.834 & 523 & 1.030 \\
\hline Pennsylyania & 126 & 33,904 & 79.825 & 113019 & 5,236 & 119,180 & 83,106 & 3.201 & 3.953 & 35,171 \\
\hline Subdistrict IC Total & 2,898 & 92,391 & 179,518 & 271,909 & 10,120 & 284,926 & 828,236 & 9,402 & 4,893 & 1,933 \\
\hline Florida & 24 & 2.350 & 38,010 & $40.4 \times 0$ & 1.017 & 41.461 & 174.921 & $11 \%$ & 1.523 & 126 \\
\hline Goorgie & 89 & 6.382 & 18.271 & 24,660 & 1.223 & $25,9 / 2$ & 144,981 & 301 & $21 ?$ & 298 \\
\hline North Carolina & 127 & 37,331 & 24,934 & $62.266)$ & 4.452 & 66.845 & 286.587 & 3.688 & 120 & \\
\hline South Carolina & 31 & 11,990 & 20,333 & 32,327 & 245 & $32.60 ?$ & $76,85 ?$ & 2.500 & $16 ?$ & 194 \\
\hline Virgınia $\ldots . .$. & 151 & 24,492 & 13.545 & $38,03 t$ & 3,119 & 41,301 & 123,605 & $1.1 \%$ & B) 5 & 1.326 \\
\hline West Virginua & $2,4 / 5$ & 9,1346 & 64,353 & 14.190 & 63 & 16.131 & 22,290 & 1.551 & $1.9 / 2$ & \\
\hline PAD Dlstrict II Total & 27,356 & 168,887 & 315,614 & 684,501 & 2,163 & 714,020 & 347,094 & 11,149 & 81,800 & 107,634 \\
\hline lilinois .... & 2.926 & 15.410 & 81,122 & 96,573 & 40 & 99.499 & 8,451 & $1,3 w 3$ & 1.680 & 1358 \\
\hline Indana .... & 5.108 & 12.278 & $B 1,36,3$ & $0364 ?$ & 5,4 & 96,805 & 105,343 & 1.054 & 1.330 & 59.490 \\
\hline lowa & 1,312 & 1.125 & 8. 428 & 15,553 & & 16,865 & 2,053 & 51 & $15 ?$ & \\
\hline Kansas .. & 811 & 3,1380 & 4,412 & B.?9? & 336 & 9.505 & 5.1013 & 11 & 4,734 & 1.550 \\
\hline Kentucky & 1.719 & 28,897 & 120,213 & $10,3,110$ & ifj & 156.645 & 8.964 & $26 ? 2$ & 2511 & 0.919 \\
\hline Metingan & 2.170 & 18,636 & $30,3.34$ & $48,9 / 0$ & & 51,140 & 31,181 & 1.216 & 6,330 & 1.124 \\
\hline Minnesola & 6,086 & 18,913 & 10,318 & 29.230 & 289 & 35.606 & 29,379 & 14 & 858 & 12.551 \\
\hline Missoun & 1.480 & 8,399 & 16,587 & 24.945 & 255 & 26120 & $25,96 ?$ & $b \beta$ & 300 & $39 ?$ \\
\hline Nobraska & 554 & 551 & 5.219 & 5,830 & 93 & $6.4 / 6$ & 6,181 & 32 & $2 B$ & \\
\hline North Dakota & 2,015 & 324 & 1.118 & B. 101 & & 10.116 & 4,325 & 4 & 5,658 & 1492 \\
\hline Ohio $\ldots$ & 513 & 43,803 & 16.140 & $1,205,43$ & $5 / 1$ & 121632 & 56.893 & 3,839 & 4910 & 3,754 \\
\hline Oklahoma & 399 & 918 & $1 /, 3 / 4$ & 18202 & 14 & 16.106 & 24,355 & $29 ?$ & 51.268 & \\
\hline South Dakola & 927 & B4? & 8.508 & 9.350 & 129 & 10.405 & 4.615 & 11 & 19 & $\cdots$ \\
\hline Tennossoe & 174 & $2,6.6$ & 31.068 & 33635 & 335 & 34.504 & 12,241 & 325 & 1056 & 264 \\
\hline Wisconsin $\ldots$ & 1.096 & 6,285 & 10.090 & $16.3 / 5$ & $2 f_{i}$ & 17.491 & 21,116 & 188 & 594 & 4.739 \\
\hline PAD Dletrict III Total & 2,116 & 20,900 & 333,028 & 353,925 & 247 & 356,288 & 105,514 & 4,729 & 384,498 & 2,324 \\
\hline Alaberma $\ldots . . . .$. & 644 & 6,703 & $88,8 / 4$ & $95.5 / 6$ & & $96.22 ?$ & 15,739 & $1,3 / 6$ & 10.851 & \\
\hline Arkanisas & 5 & 762 & 13,669 & 14431 & 181 & 14,617 & 1.139 & 84 & 3,312 & \\
\hline I ousiana & 6 & 167 & 49.495 & 49,663 & 51 & 49.120 & 42,535 & 830 & 296.086 & \\
\hline Mississippl & 631 & 7,416 & 22.809 & $30 \times 5$ & 15 & 30.1310 & 8.133 & 436 & 11.501 & \\
\hline Now Mexico & 587 & 179 & 16.205 & 16385 & & 16,912 & 5,423 & 351 & 13,631 & \\
\hline lexas & 242 & 5,612 & 141.973 & 147.6 .45 & & 141.481 & 32.544 & 1.646 & 249.111 & 2.324 \\
\hline PAO DIstrict IV Total & 16,823 & 34,961 & 140,839 & 175,801 & 433 & 193,056 & 7,190 & 318 & 43,518 & 6,956 \\
\hline Colorado ........ & 1,988 & 21,390 & 21.713 & 49,103 & & 51,091 & 159 & 52 & 9.694 & \\
\hline luaho & 1,389 & 3,560 & B. 600 & 12. $1(x)$ & & 13556 & 331 & 11 & $6 ?$ & \\
\hline Montana & 5,643 & 1.8336 & 32,226 & 34.111 & 100 & $30,85,5$ & 3,621 & 1 & $1.06 \%$ & \\
\hline Ulan ....... & 1.452 & 1,582 & $30,3 \times n$ & $31.90 \%$ & 7 & 33,360 & 2.176 & 27 & 10.185 & 6.866 \\
\hline Wyoluing & 6,351 & 544 & 41,973 & A8.517 & 326 & 55,194 & 291 & $2 ? 7$ & 22,501 & 90 \\
\hline PAD DIstrict V Total & 12,984 & 18,887 & 263,219 & 282,106 & 9,271 & 304,361 & 156,293 & 1,475 & 61,632 & 56,992 \\
\hline Alaska & 9.184 & 1.159 & 1,401 & 8,56 & & 17.750 & 18.800 & 11 & 47.865 & \\
\hline Arizona & 1 & 175 & $53,36 ?$ & 53.536 & 0.101 & 61634 & 3,990 & 14 & $1 / 3$ & \\
\hline Callorna & $45 ?$ & 1.089 & $5,5,111$ & 56,800 & 939 & 58,191 & 50.961 & 5,838 & 12.248 & 21,069 \\
\hline Hawh" & & 1,628 & 3,699 & $5,3 \% 7$ & & 5321 & 11,828 & 9 & 1.043 & $31,63 ?$ \\
\hline Nevada & 2.930 & 1,064 & $99,1 / 3$ & 100.23 & 20 & 103,187 & 3.392 & 389 & 189 & \\
\hline Orezon & 189 & $3,131 ?$ & 72400 & 26.212 & & 20,401 & $21,35 ?$ & 309 & 3 & \\
\hline Weshington & 229 & 9.961 & 21,461 & 31,429 & 205 & 31.861 & $3 / .969$ & 15,6 & $11 ?$ & 4.291 \\
\hline
\end{tabular}

Dasties ( ) : No data roported

Note lotals may not equal sum of components due to independent rounding

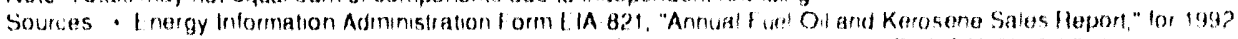

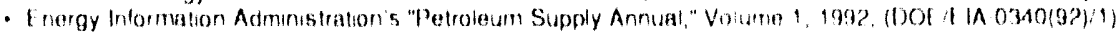


Table 22. Adjusted Sales for Farm Use: Distlliate Fuel Oll and Kerosene; Adjusted Sales for Electric Uillity Use: Dlstillate Fuel Oll and Residual Fuel OII, 1992 (Thousand Gallons)

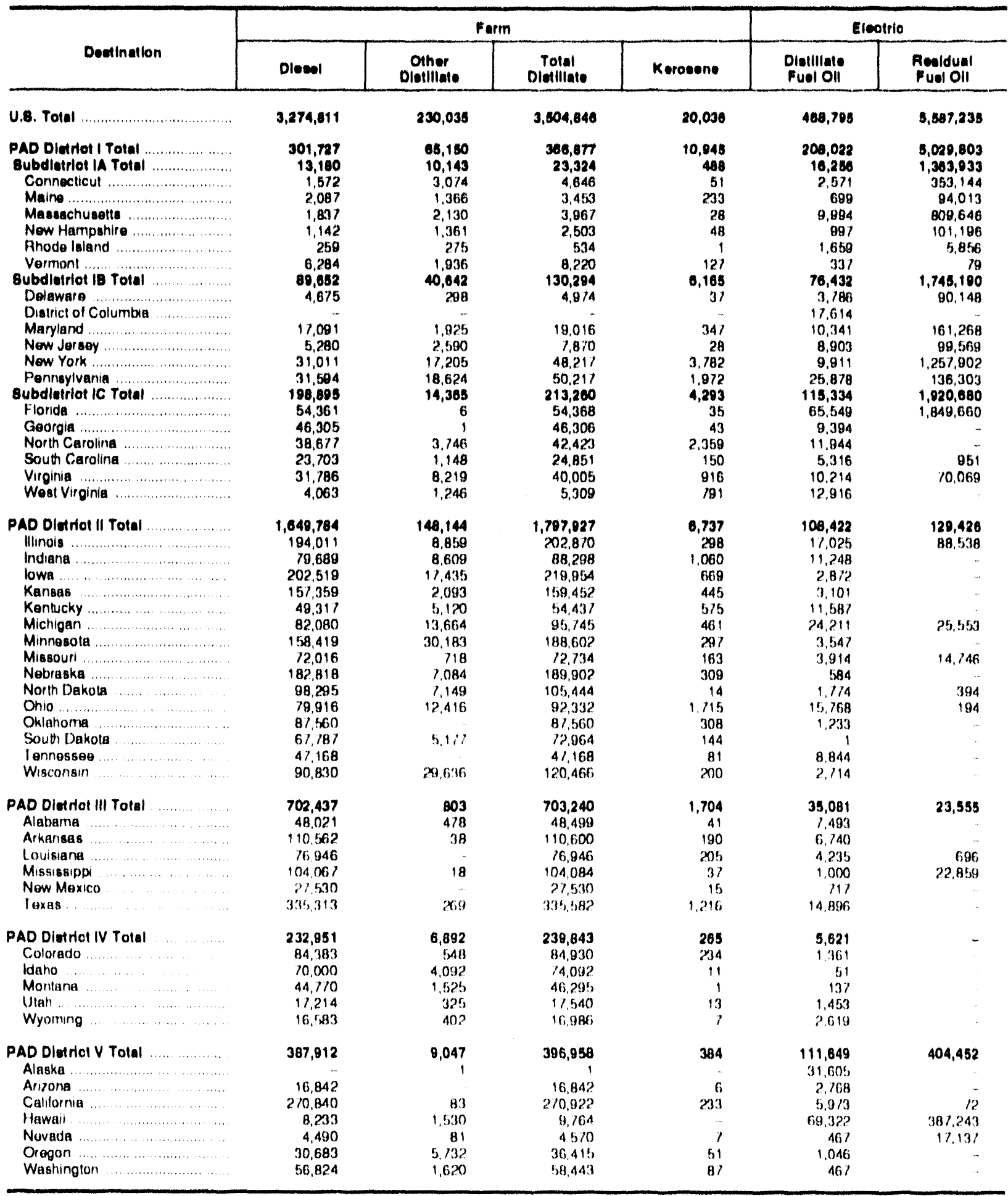

Dashes $(1)=$ No data reported

Note: Totals may not equal sum of compononts due to independent rounding

Sources: - Energy Inlormation Administration form E. IA. B21. "Annual Fuot Oil and Kerosene Sales Heport," for 1992

- Electric Utility data are besod on data obtained on Form E.IA.759, "Monthly Powor Plant Ruport." and Form F ERC 423, "Monthly Heport of Cost and Quality of Fugis for Elocitric Plants."

- Energy Information Administration's "Potroloum Supply Annual," Volume 1, 1992, (DOE LIA 0340(92)/1) 
Table 23. Adjuated Sales for Traneportation Use: Dietillate Fuel Oll and Residual Fuel Oll, 1982 (Thousand Gallons)

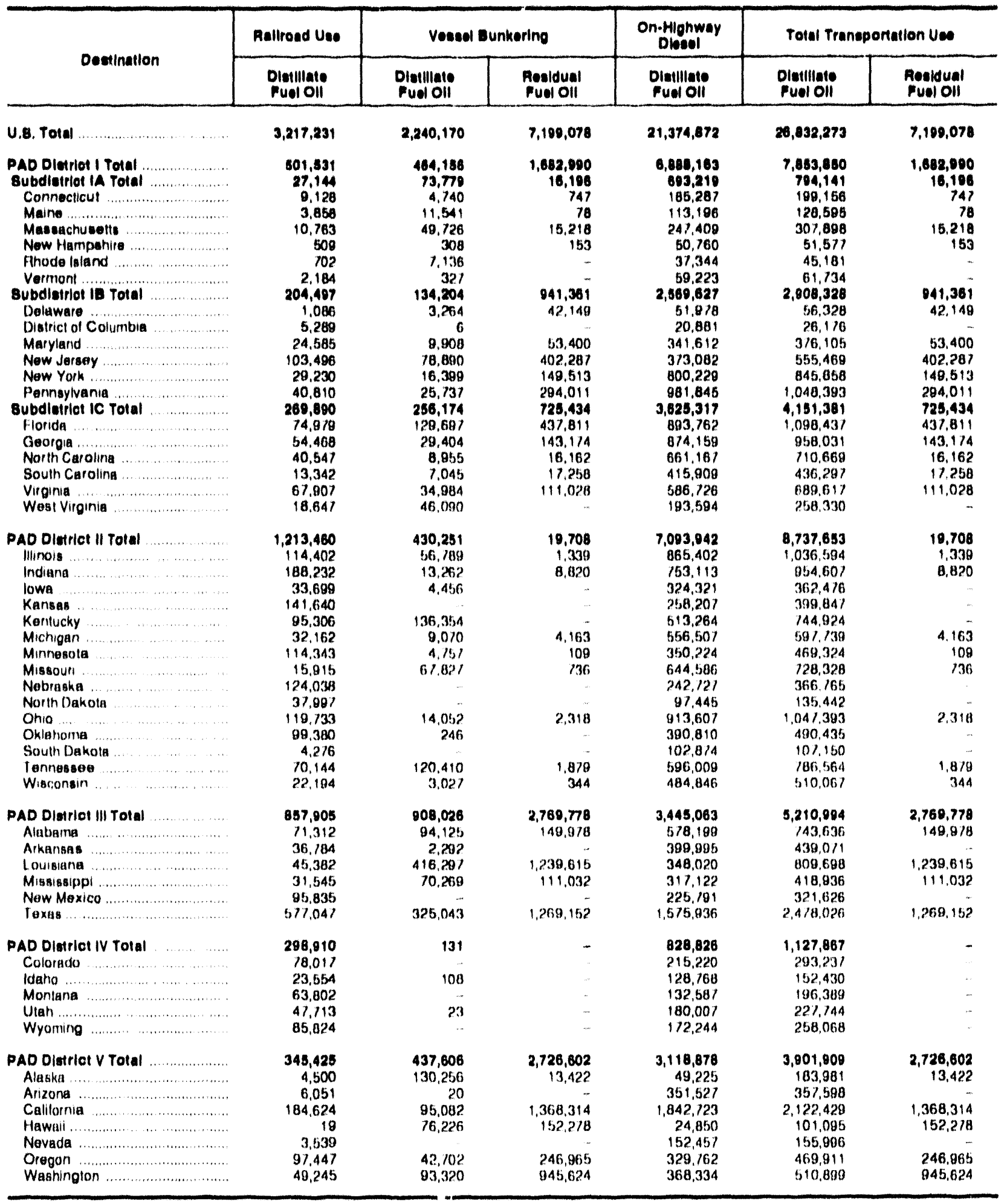

Dashes (-) = No data reported

Note: Totals may not equal sum of components due to independent rounding

Sources: - Energy Intormation Administration Form E.A. 821, "Annual Fuel Oil and Kerosane Sales Repont," tor 1992

- For 1898 through 1990, railroad data for distillate fuel oil are based on the Association of American Railroads' statistics lor railroads of Class I and Amtrak Companies. ElA-821 suney results are used lor 1981 and 1992.

- On-Highway Diesel data are Federal Highway Administation stalistics ol highway special fuels use.

- Energy Information Administration's "Petroloum Supply Annual," Volume 1, 1092, (DOE/F:IA-0340(92)/1). 
Table 24. Adjusted Sales for Milltary, Otf-HIghway, and All Other Uses: Distlliate Fuel Oll, Resldual Fuel Oll, and Kerosene, 1892 (Thousand Gallons)

\begin{tabular}{|c|c|c|c|c|c|c|c|c|c|c|}
\hline \multirow{2}{*}{ Deatination } & \multicolumn{4}{|c|}{ Militan } & \multicolumn{3}{|c|}{$\begin{array}{l}\text { OH.HIghway Diecal } \\
\text { Dietlilate Fuel OII }\end{array}$} & \multicolumn{3}{|c|}{ All Other } \\
\hline & Dionel & $\begin{array}{c}\text { Oinor } \\
\text { Dietiliate }\end{array}$ & $\begin{array}{c}\text { Total } \\
\text { Distilinte }\end{array}$ & $\begin{array}{l}\text { Rouldual } \\
\text { Pual Oll }\end{array}$ & Construation & Other & Total & $\begin{array}{l}\text { Ditulliate } \\
\text { Fual Oll }\end{array}$ & $\begin{array}{l}\text { Rosidual } \\
\text { Puol Oll }\end{array}$ & Keresene \\
\hline U.8. Total ............................ & 848,673 & 101,008 & $8 s 0,178$ & 100,684 & $1,814,203$ & 204,884 & $1,730,000$ & 7,075 & 9,446 & 0,392 \\
\hline 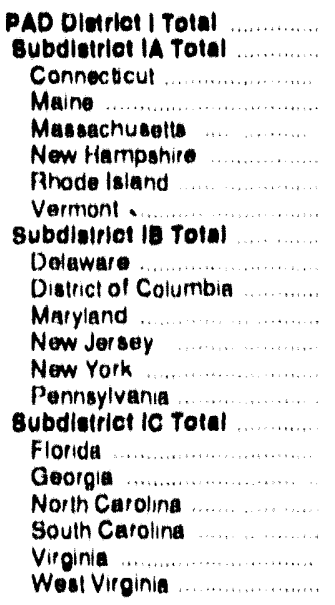 & $\begin{array}{r}31,109 \\
3,421 \\
244 \\
1,677 \\
965 \\
278 \\
32 \\
25 \\
28,348 \\
407 \\
999 \\
3,627 \\
11,086 \\
2,934 \\
1,094 \\
21,400 \\
1,493 \\
5,230 \\
976 \\
1,322 \\
6,251 \\
128\end{array}$ & $\begin{array}{r}71,794 \\
11,863 \\
4,817 \\
875 \\
2,207 \\
1,415 \\
2,520 \\
19 \\
33,822 \\
1,280 \\
1,889 \\
17,403 \\
3,821 \\
3,719 \\
2,409 \\
20,370 \\
508 \\
2,869 \\
5,703 \\
1,588 \\
14,817 \\
874\end{array}$ & $\begin{array}{r}122,023 \\
18,274 \\
5,061 \\
2,151 \\
3,172 \\
1,693 \\
2,551 \\
44 \\
30,870 \\
1,688 \\
5,887 \\
21,230 \\
20,909 \\
6,653 \\
3,503 \\
47,779 \\
8,001 \\
8,118 \\
6,679 \\
2,910 \\
21,061 \\
1,003\end{array}$ & $\begin{array}{r}87,020 \\
18,602 \\
1,127 \\
6,542 \\
3,207 \\
5,026 \\
2.513 \\
188 \\
37,910 \\
1.856 \\
302 \\
15,798 \\
6,640 \\
8,687 \\
2,628 \\
30,300 \\
2.542 \\
11,798 \\
5.380 \\
10,789 \\
-\end{array}$ & $\begin{array}{r}300,848 \\
12,848 \\
9,659 \\
4,726 \\
14,951 \\
5,734 \\
2,323 \\
5,162 \\
172,293 \\
2,816 \\
458 \\
18,961 \\
22,216 \\
41,826 \\
85,914 \\
146,007 \\
52,683 \\
28,859 \\
21,126 \\
13,304 \\
22,433 \\
7,601\end{array}$ & $\begin{array}{r}39,800 \\
3,530 \\
1 \\
3,759 \\
242 \\
933 \\
15 \\
589 \\
5,842 \\
156 \\
10 \\
10 \\
1,645 \\
284 \\
3,146 \\
27,870 \\
1,980 \\
15,830 \\
4,132 \\
3,827 \\
1,545 \\
464\end{array}$ & $\begin{array}{r}400,104 \\
46,084 \\
9,660 \\
8,484 \\
15,194 \\
6,667 \\
2,338 \\
5,741 \\
176,134 \\
2,972 \\
458 \\
18,970 \\
23,863 \\
42,210 \\
89,661 \\
173,890 \\
54,663 \\
44,780 \\
25,258 \\
17,132 \\
23,978 \\
8,065\end{array}$ & 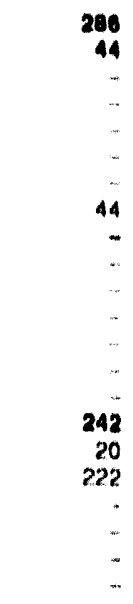 & 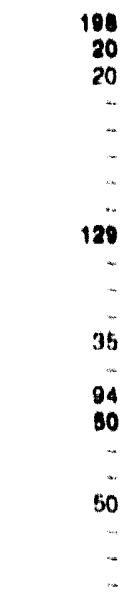 & $\begin{array}{r}3,607 \\
170 \\
11 \\
94 \\
63 \\
263 \\
2 \\
44 \\
2,210 \\
11 \\
4 \\
101 \\
509 \\
236 \\
692 \\
1,001 \\
177 \\
62 \\
69 \\
250 \\
255 \\
168\end{array}$ \\
\hline 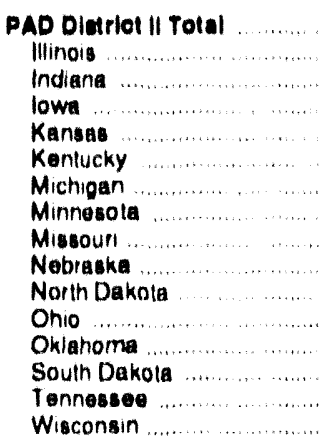 & $\begin{array}{r}66,901 \\
1,393 \\
5,359 \\
6,023 \\
18,809 \\
7,537 \\
6,261 \\
615 \\
2.372 \\
318 \\
569 \\
0,311 \\
815 \\
324 \\
4,239 \\
1,857\end{array}$ & $\begin{array}{r}10,801 \\
552 \\
1,911 \\
451 \\
2,511 \\
636 \\
3,855 \\
29 \\
22 \\
231 \\
7,952 \\
1,605 \\
74\end{array}$ & $\begin{array}{r}83,702 \\
1,045 \\
1,271 \\
6,923 \\
19,260 \\
10,041 \\
6,897 \\
815 \\
6,228 \\
318 \\
591 \\
9.54 ? \\
815 \\
5,276 \\
5,844 \\
1.931\end{array}$ & $\begin{array}{l}- \\
\cdots \\
- \\
\cdots \\
\cdots \\
\cdots \\
\cdots \\
\cdots \\
\cdots \\
\cdots \\
\cdots\end{array}$ & $\begin{array}{r}448,686 \\
52,200 \\
38,961 \\
20,307 \\
15,963 \\
27,754 \\
37,403 \\
33,389 \\
35,890 \\
8,984 \\
2,017 \\
48,258 \\
18,175 \\
7,120 \\
69,668 \\
33,584\end{array}$ & $\begin{array}{r}26,963 \\
576 \\
3.028 \\
4,113 \\
1,303 \\
1,181 \\
3,628 \\
1,713 \\
1,167 \\
1,100 \\
150 \\
1,572 \\
169 \\
1,114 \\
1,645 \\
3,103\end{array}$ & $\begin{array}{r}476,849 \\
52,776 \\
41,089 \\
24,421 \\
17,266 \\
20,535 \\
41,231 \\
35,103 \\
37,057 \\
10,084 \\
2,166 \\
49,831 \\
18,944 \\
8,234 \\
71,313 \\
36,698\end{array}$ & $\begin{array}{r}807 \\
203 \\
-21 \\
21 \\
10 \\
28 \\
1 \\
120 \\
- \\
- \\
418\end{array}$ & 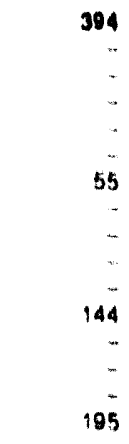 & $\begin{array}{r}1,437 \\
273 \\
136 \\
27 \\
148 \\
252 \\
41 \\
27 \\
37 \\
2 \\
1 \\
173 \\
103 \\
114 \\
114 \\
105\end{array}$ \\
\hline 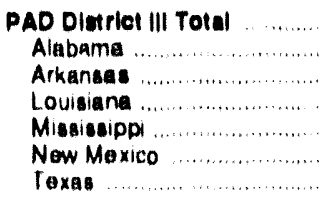 & $\begin{array}{r}239,083 \\
5,966 \\
577 \\
8,538 \\
19,255 \\
3,695 \\
201.021\end{array}$ & $\begin{array}{r}3,202 \\
544 \\
- \\
- \\
- \\
-\end{array}$ & $\begin{array}{r}242,345 \\
6,511 \\
577 \\
8,538 \\
19.255 \\
3,695 \\
203,768\end{array}$ & $\begin{array}{l}- \\
- \\
- \\
-\end{array}$ & $\begin{array}{r}388,703 \\
51,970 \\
45,503 \\
51,622 \\
18,490 \\
20,901 \\
200,301\end{array}$ & $\begin{array}{r}82,288 \\
10,168 \\
10,429 \\
4,185 \\
16,130 \\
1,263 \\
30,479\end{array}$ & $\begin{array}{r}471,048 \\
71,138 \\
55,932 \\
55,807 \\
35,221 \\
22,164 \\
230,787\end{array}$ & $\begin{array}{r}5,841 \\
535 \\
5,007 \%\end{array}$ & $\begin{array}{l}- \\
- \\
-\end{array}$ & $\begin{array}{r}1,085 \\
68 \\
112 \\
85 \\
168 \\
30 \\
593\end{array}$ \\
\hline $\begin{array}{l}\text { PAD Dietriot IV Total } \\
\text { Colorado } \\
\text { Idaho } \\
\text { Montana } \\
\text { Utah } \\
\text { Wyorning }\end{array}$ & $\begin{array}{r}17,858 \\
7,865 \\
4,841 \\
1,205 \\
1,884 \\
1,863\end{array}$ & $\begin{array}{r}381 \\
282 \\
90 \\
\cdots\end{array}$ & $\begin{array}{r}18,239 \\
8,147 \\
4,841 \\
1,304 \\
1,084 \\
1,963\end{array}$ & $\begin{array}{l}- \\
- \\
- \\
-\end{array}$ & $\begin{array}{r}103,831 \\
36,668 \\
9,619 \\
18,622 \\
20,310 \\
18,612\end{array}$ & $\begin{array}{r}10,458 \\
337 \\
5,957 \\
2,892 \\
428 \\
843\end{array}$ & $\begin{array}{r}114,200 \\
37,005 \\
15,576 \\
21,514 \\
20,738 \\
10,465\end{array}$ & $\begin{array}{r}93 \\
28 \\
65 \\
- \\
-\end{array}$ & $\begin{array}{l}42 \\
42 \\
4\end{array}$ & $\begin{array}{r}42 \\
29 \\
4 \\
6 \\
1 \\
2\end{array}$ \\
\hline 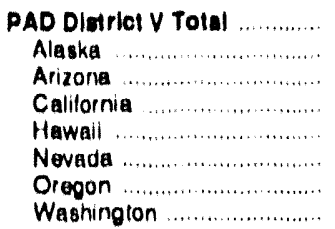 & $\begin{array}{r}173,892 \\
16,838 \\
1,468 \\
138,145 \\
8,021 \\
1,665 \\
1,682 \\
5,772\end{array}$ & $\begin{array}{r}9,377 \\
2,740 \\
1,482 \\
-7 \\
975 \\
124 \\
4,054\end{array}$ & $\begin{array}{r}162,080 \\
10,579 \\
1,469 \\
139,628 \\
8,021 \\
2,640 \\
1,806 \\
0,827\end{array}$ & $\begin{array}{r}13,663 \\
6,- \\
9,835 \\
8,838 \\
61 \\
3,830\end{array}$ & $\begin{array}{r}211,049 \\
11,331 \\
21,106 \\
112,254 \\
9,177 \\
17,176 \\
9,767 \\
29,638\end{array}$ & $\begin{array}{r}65,928 \\
5,953 \\
1,115 \\
12,801 \\
- \\
315 \\
22,760 \\
22,982\end{array}$ & $\begin{array}{r}276,977 \\
17,284 \\
22,221 \\
125,056 \\
9,177 \\
18,091 \\
32,527 \\
52,620\end{array}$ & $\begin{array}{r}48 \\
10 \\
10 \\
39 \\
\cdots\end{array}$ & $\begin{array}{r}8,814 \\
01 \\
0,123 \\
\ldots \\
\ldots\end{array}$ & $\begin{array}{r}161 \\
3 \\
1 \\
128 \\
5 \\
5 \\
23 \\
2\end{array}$ \\
\hline
\end{tabular}

Dashes (.) No data reported.

Note: Totals may not equal sum of components due to independent rounding

Sources: - Energy Inlormation Administration Form E:|A-821, "Annual Fuel Oill ana Kerosene Sales Heport," 10 r 1992

- For 1988 through 1890, military data for residual fuel oil are based on the Delense Fuel Supply Center's "DE IS Report." EIA 821 suney results are used for 1891 and 1982.

- Energy Information Administration's "Petroleum Supply Annual," Volume 1, 1992, (DOE/EIA 0340(92)/1) 

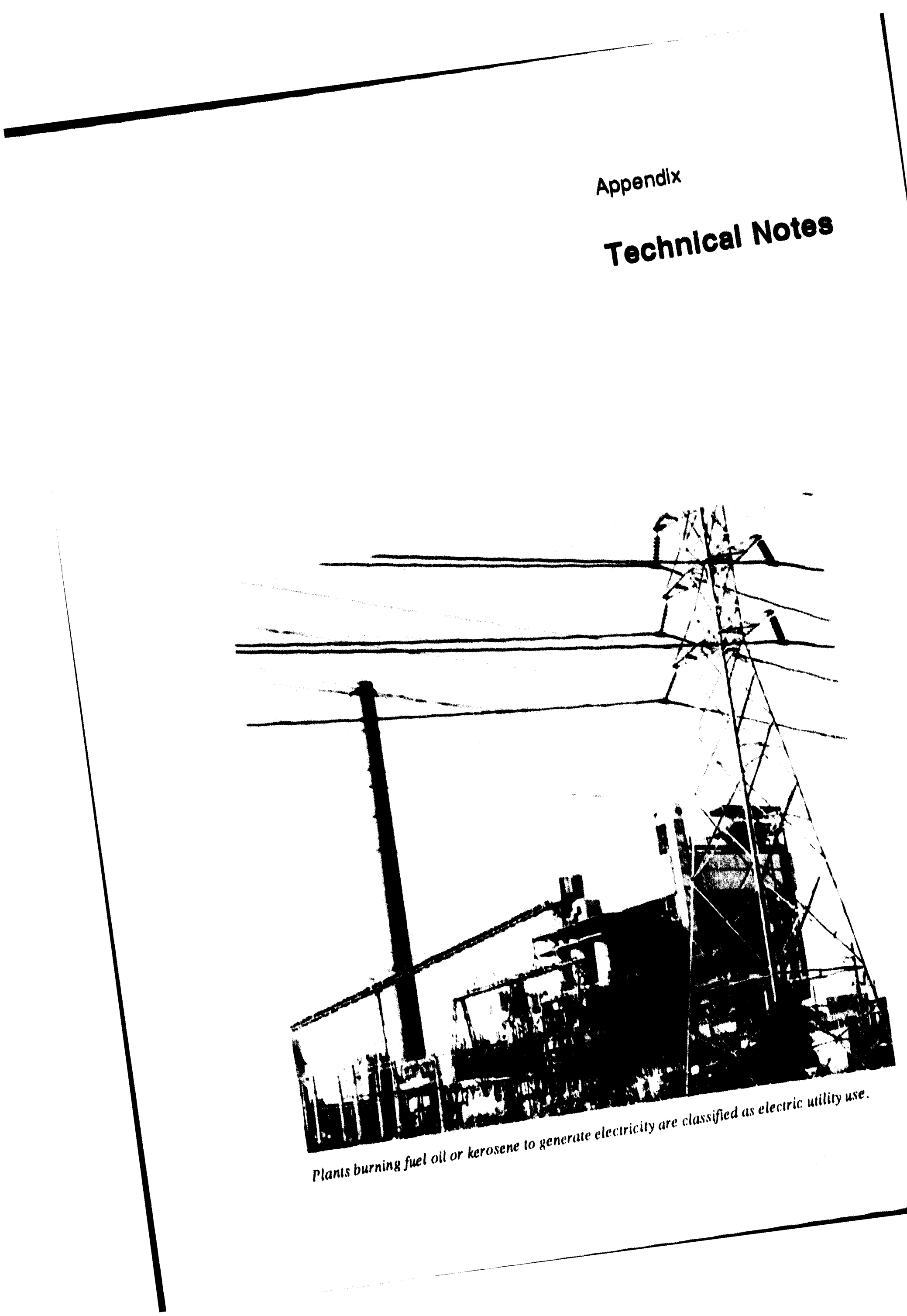


\section{Technical Notes}

\section{Note 1: ElA-821: Annual Fuel Oll and Kerosene Sales Report, 1992}

\section{Background}

The ELA.821, "Annual Fuel Oil and Kerosene Sales Report" survey was implemented to meot Energy Information Administration (EIA) logislativo mandates and data usor needs. Form EiIA.821 is used to gather data on the sales to end users of distillnte fuel oil, residual fuel oil, and kerosene. The dath aro used to determine the level of demand by end-use category and product at the State, regional, and national levels. This mandatory report is nuthorized by the Federnl Energy Adminiatration Act of 1974 (P. L. 93-276).

The predecessor survoy to the BIA.821 wiss the EIA.172, "Sules Report of Fuel Oil and Keronene" survey. Tho ELA.172 was conducted for roference years 1979 through 1982. Due to a serious doteriorn. tion in the sample of respondents during the 4 -yenr period, the Form EIA-172 data were not collected for 1983. Instend, estimates for 1983 were published and a now sample of respondonts was designed and solected from an improved sampling frame. The BIA 821 survey commenced with reference year 1984.

\section{Discussion of Sampling Frame}

The target population for the fuel oil and kerosene sules survey is the universe of compnnies that sell fuel oil and kerosene to end users.

The EIA-863 data base provided the sumpling frame for the 1992 EIA-821 survey. The EIA-863, "Petroloum Product Sales Identification Survey," was mailed to approximatoly 27,000 companios in January 1992 to collect 1991 State-level sales volume dats for $\mathrm{N}_{0} .2$ distillate, residual fuel, and motor gasoline. Compn. nies also indicated if they sold kerosene. 'The No, 2 distillate data were further identified by rosidential and nonresidential ond use, and non-end-use sales; the residual and motorgasoline dnta were identified by end use and non-end-use sules. The mailing list for the EIA-863 survey was constructed by merging and unduplicating the 1988 frame file and more than 61
State and commercial lists. In addition, company/ State-level volunies for distillate fuel oil, residual fuel oil, and motor gasoline from the 1991 EIA.821 survoy were merged with the EIA 863 to yield a combined data Ale. The tranaformed and edited vernion of the frame was then used to desigm and select the 1992 ELA-821 sample.

It should be noted that truck stops solling No. 2 diesel fuel were not specifically included in the E.A.863 survey. Therefore, the EIA-821 survey does not include all sales of No. 2 distillate through retnil truck stops. Llowover, to compensate for this shortage in coverage, data from the Federal Highway Administration are presented in the Lables for on-highwiy diesel sules.

The anmpling frame also lacked full coverage of kero. sene retailers, many of whom are hardware stores and service stations. To account for all kerosene volumes supplied to tho marketplaco, aguregate reported kero. sene volumes are set equal to the products supplied volumes publishod in the Potroleum Supply Annual.

\section{Discusslon of the Sample Design}

The purpose of the bild.821 sumple design was to produce Stute lovel estimutes of totul sules for the following five Lurget variables: (1) residential No, 2 distillate, (2) other rotuil No, 2 distillatu, (3) wholesule No. 2 distillate, (4) retail residunl, and (5) wholesule residual. A separate samplo was initinlly designed for onch target varinble.

Companies were divided into two basic types of struta: cortuinty and noncertainty. Compuniess solected with crertuinty wore:

- all refiners and gas plant operilors

- companies doing business in four or more States

- companies accounting for 5 percent or more of the distillate or residual fuel oil volume for any target variable or particular ond-use category sold in $n$ Stute.

- companies necounting for 5 percent or more of the kerosene volume sold in a State in an enrlier FiA.821 survey. 
- augmented frame units, e.g., vesmel bunkering, for which no attribute data were nvailable.

Firms determined not to be selling distillate or residual fuel oil or kerosene were removed from the frame file. Volumes for nonrespondents to the E.IA 863 who had reported in the previous frame survey or in a sumple survey were imputed. The imputed nonrespondents and the noncertainty compunies were stratified by sales volume for ench target variable. St rata bounda ries were determined using the Dalenius-Hodges procedures, allowing the number of strate and the strita boundaries to vary by Sute. Nonrespondents for whom no previous information was available were classified in a separnte stratum and sampled with reduced probabilities of selection.

Neyman allocution was used to allocate the number of companies required for ench struta to ohtain the reguired levels of accuracy: a 2.5 percent coefficient of varintion for residual fuel oil and a 5 percent coeflicient of variation for distillate fuel oil. Sample solections were then performed simuluneously from the same random list for the five samples to produce " final link eil sumplo of approximately 4,600 companies. I'Tis method reduced the totul survey sumple size whilo. improving volume estimates. Selected compminies were asked to report sales by end use centegories for distillate and residual fuel oil and kerosene

\section{Imputation and Estimation}

Sursey dente gathered from the respondents mas contuin incomplete reporting, total nomresponse, or values thent finil aditing. Missing dentu are estimuted, or implicitly imputed for, using the serutu means and treated the same as reported data. The weiphterd estimute is defined an follows

$$
\hat{v} \geq_{n} N_{h}\left(\geq, w_{h}, v_{h}, L_{1} w_{h}\right)
$$

where:

$$
\begin{aligned}
& \hat{v} \quad \text { total estimuted volume. } \\
& \text { In denotes summation over strata, } \\
& N_{h} \quad \text { stratum population for stratum h, } \\
& L_{1} \text { denotes summation over units within } \\
& \text { strutum h, } \\
& V_{h} \text {, volume reported for unit } i \text { in strutum } h \text {, } \\
& W_{h} \text {, woight attathed to unit i in strutum } h \text {. }
\end{aligned}
$$

Whare: $W_{h}$ is inversoly proportional to the probubility of inclusion in the linked snmple.
The degree of impulution by product and end use at the U.S. total level ranged as follows for the 1992 EIA.821 dats: distillate, 0.8 percent for railrond use to 9.6 purcent for all other uses; residual fuel oil, 0.0 percent for oil company use to 5.8 percent for military use; and kerosenc, 1.0 percent for wll other uses to 2.6 percent for furm use.

\section{Collectlon Methods}

The FiLA 821 form was mailed in January 1993 to all companies melected for participation in the 1992 refer. ence yenr survey. The comploted form wis due to BIA on March 1. 1998. A second request was sent by cortified mail to nonrespondents approximately i month afler the filing dendline. Follow up telephone culls were made to collect outstanding date and to verify reported datu. Jatte sulamissions and re submis. sions were processed when received

\section{Data Processing}

As ViA 821 forme were received, they were logged into III nutemuted Survey ('ont rol File which maintenined (ompuny identificution und survey form status infor. mation for ench company nelected to participate in the

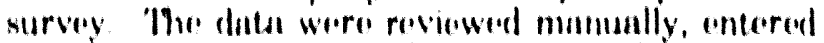
onto the computer filess, and then processed through an automuted edit program which detected missing data

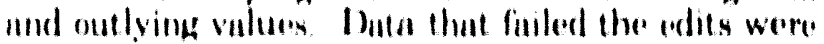
reasolved through telephome calls to the deta reporters ind corrections were entered ontes the computer files Prolimimary estimutes were genternted and processed through a series of validation procedures to flage and restify potontiul misreportings of dentes statisticol reports, including publiciotion bables, were produced

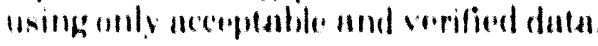

\section{Note 2: Rellability of the Data}

Two types of errors ure associnted with datu produced from in sumple survey sumplitug errors and nonsum. pling errors. Sumplinge errors accur becouse the restimutes are bused on a sample ruther then on a crinsus. The particular sample used for the F.IA. 821 survey is cone of a large number of sumples of equal size which could have been selected from the sampling frume using the same sumple design Fach of these semples would produce a diflerent estimnte. If the estimates were averaged over all possible sumples, the result would be the sume as the estimnte derived from a census of the sumpling frume. The sumpling cerror is "I monsure of variability among the estimutes from all possible samples andi, thus, is a mensure of the 
precision with which an estimate from a particular sample approximates the results of a census.

Nonsampling errors and possibly biases can arise from a number of sources: (1) inability to obtain information about all cases in the sample, (2) response errors, (3) differences in the interpretation of questions or definitions, (4) mistakes in recording or coding of the data obtained, and (5) other errors of collection, response, coverage, and estimation for missing data. Bias is the difference between the average of the estimates over all possible samples of the same size and design, and the true value being estimated. It is not possible to estimate bias using the results of one sample.

Data obtainied from alternate sources are not subject to sampling errors, but may be subject to nonsampling errors, the magnitudes of which are unknown. For example, kerosene estimates may have more nonsampling errors due to a lack of coverage of kerosene retailers. Nonsampling errors for survey estimates and estimates adjusted to alternate sources cannot be determined, but attempts are made throughout survey processing to minimize this type of error.

Data in Tables 1 through 12 are based on survey data which are subject to sampling errors. Coefficients of variation, which are estimates of sampling errors, are presented for the retail target variables in the following table for the 1992 survey. The coefficients of variation (CV) were estimated by:

$c V(\hat{V})=\frac{\sqrt{V A R(\hat{V})}}{\hat{V}}$ where:

$$
\begin{aligned}
& V A R(\hat{V})=\sum_{h} n_{h}\left(1-\frac{n_{h}}{N_{h}}\right) S_{h}^{2} \\
& S_{h}=\frac{\sum_{i=1}^{n h} W_{i}^{2} V_{i}^{2}+\bar{V} \sum_{i=1}^{n h} W_{i}^{2}-2 \bar{V}_{h} \sum_{i=1}^{n h} W_{i}^{2} V_{i}}{n_{h}-1}
\end{aligned}
$$

$$
V_{h}=\frac{\sum_{i=1}^{n h} W_{i} V_{i}}{\sum_{i=1}^{n h} W_{i}}
$$

$\hat{V}=$ total estimated volume,

$N_{h}=$ stratum population for stratum $h$,

$n_{h}=$ number of sample units in stratum $h$,

$V_{i}=$ volume for unit $i$,

$W_{i}=$ weight for unit $i$.

Response rates also offer some indication of the reliability and comprehensiveness of survey results. For the 1992 EIA.821 survey, the overall response rate the number of submissions received, divided by the number of submissions solicited and expected, times 100) was 91.6 percent. 
Coefficlents of Variation for Sales of Fuel Oll, 1892

\begin{tabular}{|c|c|c|c|}
\hline Oeographlc Area & $\begin{array}{c}\text { Rosidential Distillate } \\
\text { Fuel OII }\end{array}$ & $\begin{array}{c}\text { Non-Resldential } \\
\text { Rotall Diaflilate } \\
\text { Fuel oll }\end{array}$ & $\begin{array}{c}\text { Rotall } \\
\text { Realdual Puel oll }\end{array}$ \\
\hline$\ldots \ldots \ldots \ldots \ldots \ldots \ldots$ & 1.8 & 0.8 & 0.1 \\
\hline 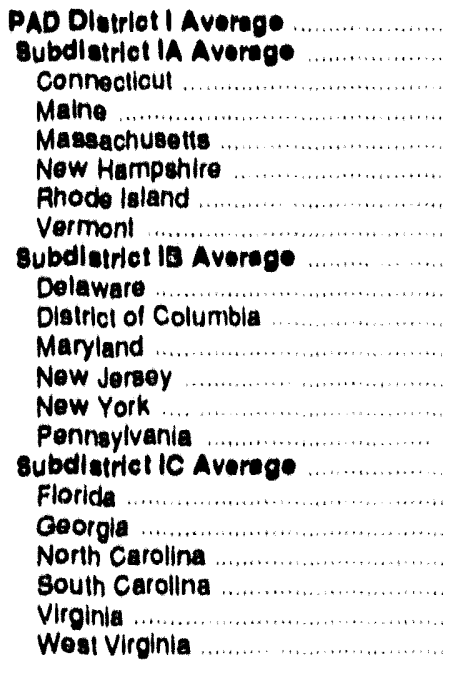 & $\begin{array}{r}1.8 \\
2.3 \\
3.0 \\
4.1 \\
3.1 \\
4.6 \\
3.8 \\
4.2 \\
2.0 \\
4.2 \\
1.8 \\
4.4 \\
3.3 \\
5.7 \\
4.2 \\
3.7 \\
11.3 \\
3.0 \\
6.8 \\
3.2 \\
5.7 \\
5.4\end{array}$ & $\begin{array}{l}1.2 \\
1.4 \\
3.0 \\
4.3 \\
1.0 \\
2.2 \\
3.8 \\
4.4 \\
1.0 \\
4.7 \\
1.1 \\
3.4 \\
4.8 \\
4.2 \\
2.8 \\
1.8 \\
3.5 \\
4.7 \\
3.4 \\
3.6 \\
5.3 \\
30\end{array}$ & $\begin{array}{l}0.1 \\
0.1 \\
0.0 \\
0.0 \\
0.1 \\
0.0 \\
0.0 \\
0.0 \\
0.1 \\
0.0 \\
0.0 \\
0.0 \\
0.1 \\
0.1 \\
0.1 \\
0.0 \\
0.0 \\
0.0 \\
0.0 \\
0.0 \\
0.0 \\
0.0\end{array}$ \\
\hline 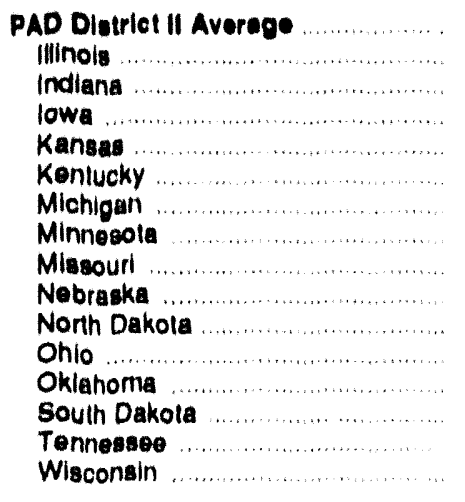 & $\begin{array}{r}2.2 \\
6.6 \\
5.5 \\
7.4 \\
178 \\
8.0 \\
4.8 \\
5.5 \\
5.9 \\
7.2 \\
60 \\
5.5 \\
60.7 \\
7.1 \\
12.5 \\
65\end{array}$ & $\begin{array}{r}1.4 \\
5.0 \\
3.6 \\
15.0 \\
4.7 \\
2 . \\
30 \\
37 \\
4.0 \\
6.0 \\
3.2 \\
28 \\
6.1 \\
4.3 \\
3.2 \\
42\end{array}$ & $\begin{array}{r}0.1 \\
0.0 \\
0.1 \\
0.0 \\
0.0 \\
0.0 \\
0.0 \\
0.0 \\
0.1 \\
0.0 \\
0.0 \\
0.0 \\
0.0 \\
16.2 \\
0.0 \\
0.0\end{array}$ \\
\hline 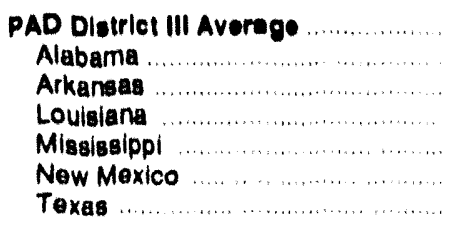 & $\begin{array}{r}28.4 \\
7.3 \\
02.5 \\
75.5 \\
30.8 \\
3.2 \\
25.6\end{array}$ & $\begin{array}{l}2.1 \\
3.8 \\
4.3 \\
3.4 \\
4.0 \\
3.1 \\
3.8\end{array}$ & $\begin{array}{l}0.1 \\
0.0 \\
0.0 \\
0.0 \\
0.0 \\
0.0 \\
0.1\end{array}$ \\
\hline 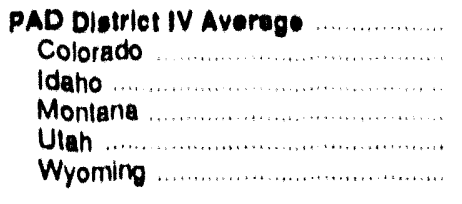 & $\begin{array}{l}4.6 \\
7.8 \\
7.5 \\
7.2 \\
4.2 \\
5.0\end{array}$ & $\begin{array}{l}1.7 \\
3.8 \\
4.3 \\
2.8 \\
2.9 \\
3.7\end{array}$ & $\begin{array}{l}0.1 \\
4.9 \\
0.0 \\
0.0 \\
0.0 \\
0.0\end{array}$ \\
\hline $\begin{array}{l}\text { PAD District V Avorage } \\
\text { Alaska } \\
\text { Arlzona } \\
\text { Callornia } \\
\text { Hawali } \\
\text { Nevada } \\
\text { Oregon } \\
\text { Washingion }\end{array}$ & $\begin{array}{l}2.8 \\
6.1 \\
1.3 \\
7.0 \\
0.0 \\
1.7 \\
6.7 \\
3.7\end{array}$ & $\begin{array}{l}2.7 \\
4.2 \\
4.6 \\
54 \\
0.2 \\
2.5 \\
4.6 \\
4.5\end{array}$ & $\begin{array}{l}0.1 \\
0.0 \\
0.1 \\
0.0 \\
0.0 \\
0.0 \\
0.0 \\
0.0\end{array}$ \\
\hline
\end{tabular}

Note: Coefilcients of variation that are less than 0.1 and greater than zero are displayed as 0.1

Sources: " Energy intormation Administration Form ElA.821, "Annual Fuel Oll and Kerosene Sales Report," lor 1982. 


\section{Note 3: Data Adjustments}

\section{Alternate Source Data}

After all preliminary tabulations were verified, comparisons were made between the survey results and available alternate source data. The following end-use categories were replaced by alternate source data at the U.S., Petroleum Administration for Defense (PAD) district, or State level:

\section{Tables 1 through 12:}

On-Highway Diesel. Distillate fuel oil by State was calculated from the Federal Highway Administration data on highway use of special fuels. Of the 1988 through 1992 special fuels, more than 98 percent is diesel.

\section{Tables 13 through 24:}

Electric Utility. National-level distillate and residual fuel oil sales for electric utility use were calculated from annual aggregations of data collected on forms EIA-759, "Monthly Power Plant Report," and FERC-423, "Monthly Report of Cost and Quality of Fuels for Electric Plants." Form EIA-759 consumption data for light oils include No. 2 fuel oil, kerosene, and jet fuel; the heavy oils include No. 4 fuel oil, residual fuel oil, nd crude oil. Using receipt data from Form FERC-423, the No. 4 fuel oil receipts were deleted from the heavy oil consumption data and added to the light oil consumption data. The resultant distillate (light oils) and residual (heavy oils) consumption data were added to the stock change of light oils and heavy oils, respectively, from Form EIA-759 to obtain the estimate of sales. Allocations at the State level were based on the EIA-821 survey.

Military. For 1987-1990, the national data on residual fuel oil issued to defense installations (Defense Fuel Supply Center's Defense Energy Information System (DFSC DEIS) Report) were allocated by State based on the EIA-821 survey. Survey results are used for 1992.

On-Highway Diesel. Distillate fuel oil by State was calculated from the Federal Highway Administration data on highway use of special fuels. Of the 1988 through 1992 special fuels, more than 98 percent is diesel.

Railroad. For 1987-1990, distillate fuel oil used by Class I railroads and Amtrak at the U.S. level was obtained from the Association of American Railroads. State-level allocations were based on the EIA-821 survey. Survey results are used for 1992.

\section{8 through 1992 Adjustments}

Kerosene volumes for 1992 in Tables 1 through 24 are adjusted at the national level to equal products supplied volumes as published in the "Detailed Statistics" section of the Petroleum Supply Annual (PSA). The 1992 survey resulted in 672,606 thousand gallons of kerosene at the national level. The products supplied volume at the national level for kerosene was 636,426 thousand gallons. Hence, the adjustment factor at the national level for 1992 was 0.95 .

For 1988 through 1991, kerosene volumes are adjusted at the national level in Tables 1 through 12 and at the PAD district level in Tables 13 through 24.

Volumes of distillate fuel oil in Tables 13 through 24 were adjusted at the PAD district level to equal products supplied volumes. The adjustment factors at the PAD district level for 1992 are as follows:

$\begin{array}{lr}\text { Distillate Fuel Oil } & \\ \text { - PAD District I: } & .83 \\ \text { - PAD District II: } & .98 \\ \text { - PAD District III: } & 1.29 \\ \text { - PAD District IV: } & 1.17 \\ \text { - PAD District V: } & .85\end{array}$

For Tables 13 through 24 , total sales at the PAD district level equal the products supplied volumes as reported in the PSA, 1988 through 1992. For example, the 1992 survey yielded a volume for distillate residential use in PAD District II of 1,007,648 thousand gallons. The total distillate volume for PAD District II was $0,527,078$ from the survey, after subtracting the volume coming from alternate sources. The product supplied total distillate volume for PAD District II was $6,375,312$ after removing alternate source volume. Hence, the adjusted 1992 estimate of distillate for residential use in PAD District II was $(6,375,312 / 6,527,078) \times 1,007,648=984,218$.

For distillate fuel oil in Tables 13 through 24, the PAI) district adjustments were made to each State and product-use category except on-highway diesel use, and electric utility use.

Volumes of residual fuel oil in Tables 13 through 24 were adjusted at the national level to equal the products supplied estimate. The 1992 survey yielded $16,199,042$ thousand gallons of residual fuel oil, while the products supplied volume at the national level was $16,816,142$ thousand gallons. Thus, the adjustment factor at the national level for 1992 was 1.10, after subtracting the volumes coming from alternate sources. For residual fuel oil, the adjustments were made to each State and end-1use category except electric utility use. 


\section{Note 4: End-Use Descriptions and Petroleum Product Definitions}

\section{End-Use Descriptions}

Residential Use. Includes sales for use in private households for space heating, water heating, cooking, etc. Sales to farm houses are reported under "Farm Use."

Commercial Use. Includes sales for use in nonmanufacturing establishments such as motels, apartment buildings, restaurants, retail stores, institutions, government buildings, schools, and laundries for space heating, water heating, and cooking. Volumes used by companies in the marketing and distribution of petroleum products are also included.

Industrial Use. Includes sales to mines, smelters, and plants engaged in producing manufactured products, processing and assembling goods, etc. Also includes space heating. Excludes oil company use, as defined below.

Oil Company Use. Includes sales to drilling companies, pipelines or other related oil companies not engaged in the selling of petroleum products. Includes fuel oil that was purchased or produced and used by company facilities for the operation of drilling equipment, other field or refinery operations, and space heating at petroleum refineries, pipeline companies, and oil-drilling companies. Oil used to bunker vessels is counted under vessel bunkering. Sales to other oil companies for field use are included, but sales for use as refinery charging stocks are excluded.

Farm Use. Includes sales for use in tractors, irrigation pumps and other agricultural machinery, as well as that used for crop drying, smudge pot fuel, space heating of buildings (including farm houses), cooking, and any other use by the agriculture industry.

Electric Utility Companies. Includes sales to electric utility companies, including that used for the generation of electricity. Volumes imported and used directly by electric utility companies are also included.

Railroad Use. Includes sales to railroads, for any use, including that used for heating buildings operated by railroads.

Vessel Bunkering. Includes sales for the fueling of commercial or private boats, such as pleasure craft, fishing boats, tugboats, and ocean "going vessels, including vessels operated by oil companies. Excluded are volumes sold to the U.S. Armed Forces.

On-Highway Diesel Use. Includes sales for use in motor vehicles.

Military Use. Includes sales to the Armed Forces, including volumes sold to the Defense Fuel Supply Center (DFSC) for use by all branches of the Department of Defense (DOD).

Off-Highway Diesel Use. Includes sales for use in:

1. Construction. Construction equipment including earthmoving equipment, cranes, stationary generators, air compressors, etc.

2. Other. Sales for off-highway uses other than construction. Sales for logging are included in this category. Volumes for off-highway diesel use by the agriculture industry are reported under "Farm Use."

All Other Uses. Sules for all other uses not included in any other end-use category are reported here.

\section{Definitions of Petroleum Products and Other Related Terms}

ASTM. The acronym for the American Society for Testing and Materials.

Distillate Fuel Oil. A general classification for one of the petroleum fractions produced in conventional distillation operations. It is used primarily for space heating, on-and off-highway diesel engine fuel (including railroad engine fuel and fuel for agricultural machinery), and electric power generation. Included are products known as No. 1, No. 2, and No. 4 fuel oils and No. 1, No. 2, and No. 4 diesel fuels.

1. No. 1 Distillate. A petroleum distillate which meets the specifications for No. 1 heating or fuel oil as defined in ASTM D 396 and/or the specifcations for No. 1 diesel fuel as defined in ASTM Specification D 975.

a. No. 1 Diesel Fuel. A volatile distillate fuel oil with a boiling range between 300 and 575 degrees Fahrenheit and used in high-speed diesel engines generally operated under wide variations in speed and load. Includes type C-B diesel fuel used for city buses and similar operations. Properties are defíned in ASTM Specification D 975. 
b. No. 1 Fuel Oil. A light distillate fuel oil intended for use in vaporizing pot-type burners. ASTM Specification D 396 specifies for this grade maximum distillation temperatures of 400 degrees Fahrenheit at the 10-percent point and 550 degrees Fahrenheit at the 90-percent point, and kinematic viscosities between 1.4 and 2.2 centistokes at 100 degrees Fahrenheit.

2. No. 2 Distillate. A petroleum distillate which meets the specifications for No. 2 heating oil or fuel oil as defined in ASTM D 396 and/or the specifications for No. 2 diesel fuel as defined in ASTM Specification D 975.

a. No. 2 Diesel Fuel. A gas oil type distillate of lower volatility with distillation temperatures at the 90-percent point between 540 and 640 degrees Fahrenheit for use in high speed diesel engines generally operated under uniform speed and load conditions. Includes Type R-R diesel fuel used for railroad locomotive engines, and Type T-T for diesel-engine trucks. Properties are defined in ASTM Specification D 975.

b. No. 2 Fuel Oil. A distillate fuel oil for use in atomizing type burners for domestic heating or for moderate capacity commercial. industrial burner units. ASTM Specification D 396 specifies for this grade distillation temperatures at the 90-percent point between 540 and 640 degrees Fahrenheit, and kinematic viscosities between 2.0 and 3.6 centistokes at 100 degrees Fahrenheit.

3. No. 4 Fuel. A fuel oil for commercial burner installations not equipped for preheating facilities. It is used extensively in industrial plants. This grade is a blend of distillate fuel oil and residual fuel oil stocks that conforms to ASTM Specification D 396 or Federal Specification VV-F-815C; its kinematic viscosity is between 5.8 and 26.4 centistokes at 100 degrees Fahrenheit. Also included is No. 4.D, a fuel oil for lowand medium-speed diesel engines that conforms to ASTM Specification D 975.

Kerosene. A petroleum distillate that boils at a temperature between 300 and 550 degrees Fahrenheit, that has a flash point higher than 100 degrees Fahrenheit by ASTM Method D 56 , that has a gravity range from 40 to 46 degrees API, and that has a burning point in the range of 150 degrees to 175 degrees Fahrenheit. Included are the two classifications recognized by
AST'M Specification D 3699: No. 1-K and No. 2-K, and all grades of kerosene called range or stove oil which have properties similar to No. 1 fuel oil, but with a gravity of about 43 degrees API and a maximum endpoint of 625 degrees Fahrenheit. Kerosene is used in space heaters, cook stoves, and water heaters and is suitable for use as an illuminant when burned in wick lamps. NOTE: Respondents to the EIA-821 survey were instructed to report all volumes in accordance with what the product was sold as, regardless of the actual specifications of that product. For example, if a No. 2 distillate was sold as a heating oil or fuel oil, the volume would be reported in the category "No. 2 Fuel Oil" even if the product conformed to the higher specifications of a diesel fuel.

PAD District. Petroleum Administration for Defense Districts

PAD District I:

Subdistrict IA. Connecticut, Maine, Massachusetts, New Iampshire, Rhode Island, and Vermont

Subdistrict IB. Delaware, District of Columbia, Maryland, New Jersey, New York, and Pennsylvania

Subdistrict IC. Florida, Georgia, North Carolina, South Carolina, Virginia, and West Virginia

\section{PAD District II:}

Illinois, Indiana, lowa, Kansas, Kentucky, Michigan, Minnesota, Missouri, Nebraska, North Dakota, South Dakota, Ohio, Oklahoma, Tennessee, and Wisconsin

\section{PAD District II I:}

Alabama, Arkansas, Louisiana, Mississippi, New Mexico, and Texas

\section{PAD IDistrict IV:}

Colorado, Idaho, Montana, Utah, and Wyoming

\section{PAI District V:}

Alaska, Arizona, California, Hawaii, Nevada, Oregon, and Washington

Residual Fuel Oils. The topped crude of refinery operations, which includes No. 5 and No. 6 fuel oils, as defined in AS'TM Specification D 396 and Federal Specification, VV-F-815C; Navy Special fuel oil as 
defined in Military Specification MIL-F-859E including Amendment 2 (NATO symbol F-77); and Bunker C fuel oil. Residual fuel oil is used for the production of electric power, space heating, vessel bunkering, and various industrial purposes.
United States. The 50 States and the District of Columbia.

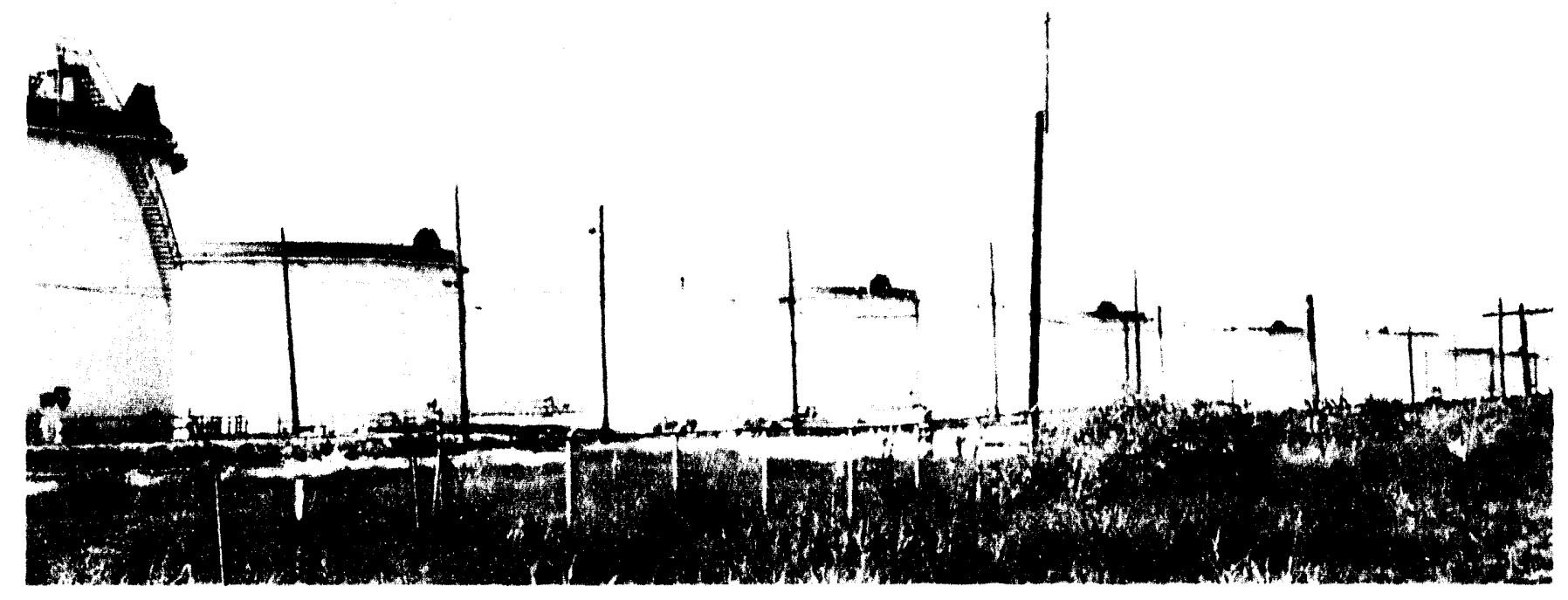

Storage and distribution of petroleum products are becoming more challenging in the 1990s due to a wide variety of new air quality regulations. 

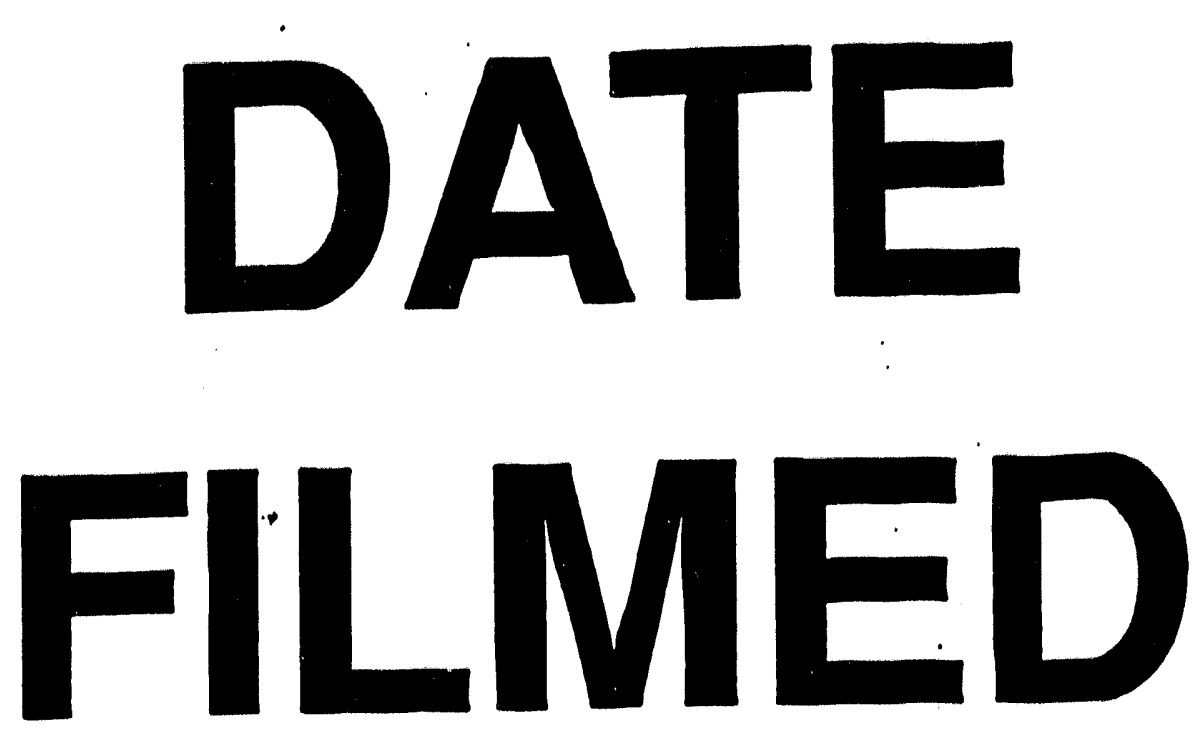

$12 / 27 / 93$
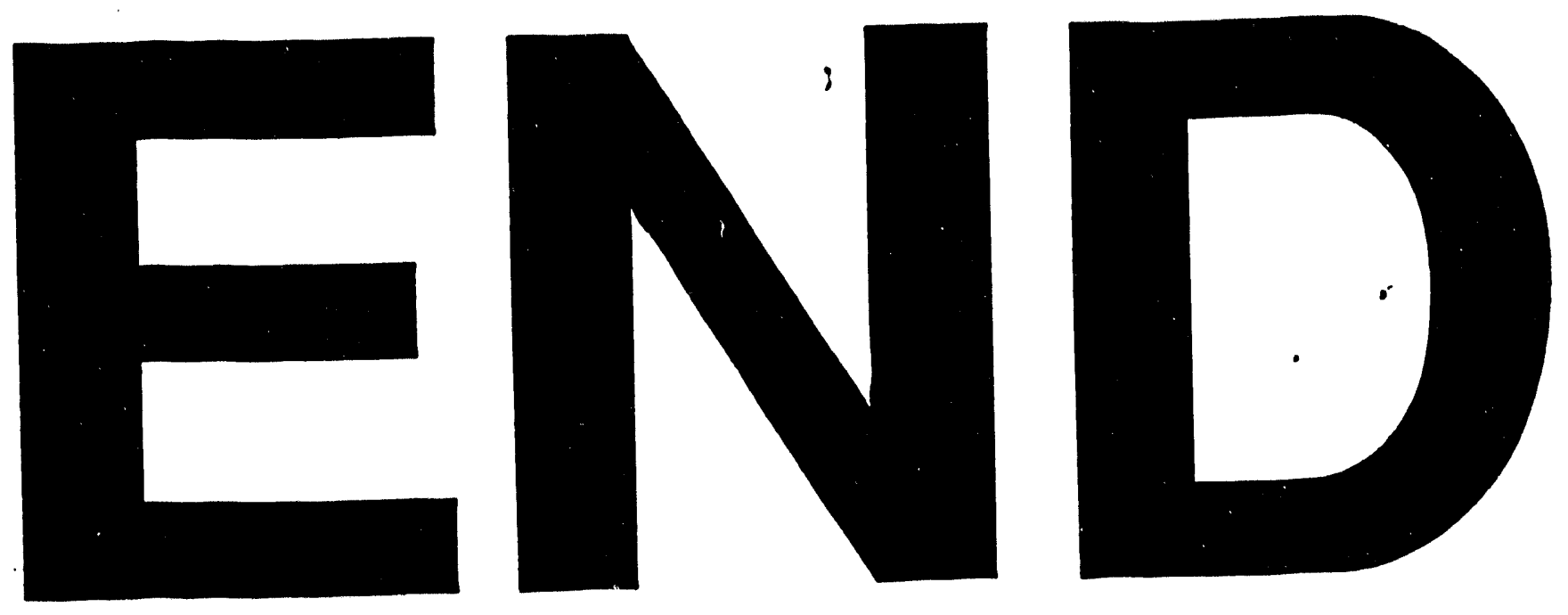


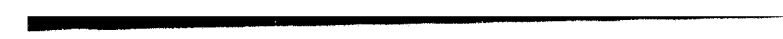

